\title{
FIRST-ORDER AND TEMPORAL LOGICS FOR NESTED WORDS
}

\author{
RAJEEV ALUR ${ }^{a}$, MARCELO ARENAS $^{b}$, PABLO BARCELÓ $^{c}$, KOUSHA ETESSAMI $^{d}$, \\ NEIL IMMERMAN ${ }^{e}$, AND LEONID LIBKIN ${ }^{f}$ \\ ${ }^{a}$ Department of Computer and Information Science, University of Pennsylvania \\ e-mail address: alur@cis.upenn.edu \\ ${ }^{b}$ Department of Computer Science, Pontificia Universidad Católica de Chile \\ e-mail address: marenas@ing.puc.cl \\ ${ }^{c}$ Department of Computer Science, Universidad de Chile \\ e-mail address: pbarcelo@dcc.uchile.cl \\ ${ }^{d}$ School of Informatics, University of Edinburgh, Edinburgh \\ e-mail address: kousha@inf.ed.ac.uk \\ ${ }^{e}$ Department of Computer Science,University of Massachusetts \\ e-mail address: immerman@cs.umass.edu \\ ${ }^{f}$ School of Informatics, University of Edinburgh, Edinburgh \\ e-mail address: libkin@inf.ed.ac.uk
}

REvision Note. This is a revised and corrected version of the article originally published on November 25, 2008.

ABSTRACT. Nested words are a structured model of execution paths in procedural programs, reflecting their call and return nesting structure. Finite nested words also capture the structure of parse trees and other tree-structured data, such as XML.

We provide new temporal logics for finite and infinite nested words, which are natural extensions of LTL, and prove that these logics are first-order expressively-complete. One of them is based on adding a "within" modality, evaluating a formula on a subword, to a logic CaRet previously studied in the context of verifying properties of recursive state machines (RSMs). The other logic, NWTL, is based on the notion of a summary path that uses both the linear and nesting structures. For NWTL we show that satisfiability is EXPTIME-complete, and that model-checking can be done in time polynomial in the size of the RSM model and exponential in the size of the NWTL formula (and is also EXPTIME-complete).

Finally, we prove that first-order logic over nested words has the three-variable property, and we present a temporal logic for nested words which is complete for the two-variable fragment of first-order.

1998 ACM Subject Classification: F.1.1, F.3.1, F.4.1.

Key words and phrases: Nested Word, Temporal Logic, First-Order Expressive Completeness, ThreeVariable Property, Nested Word Automata, Model Checking.

님

LOGICAL METHODS IN COMPUTER SCIENCE
DOI:10.2168/LMCS-4 (4:11) 2008
(C) R. Alur, M. Arenas, P. Barceló, K. Etessami, N. Immerman, and L. Libkin (c) Creative Commons 


\section{INTRODUCTION}

An execution of a procedural program can reveal not just a linear sequence of program states encountered during the execution, but also the correspondence between each point during the execution at which a procedure is called and the point when we return from that procedure call. This leads naturally to the notion of a finite or infinite nested word (see [4, 3, 2]). A nested word is simply a finite or $\omega$-word supplied with an additional binary matching relation which relates corresponding call and return points (and of course satisfies "well-bracketing" properties). Finite nested words offer an alternative way to view any data which has both a sequential string structure as well as a tree-like hierarchical structure. Examples of such data are XML documents and parse trees.

Pushdown systems (PDSs), Boolean Programs, and Recursive State Machines (RSMs), are equivalent abstract models of procedural programs, with finite data abstraction but unbounded call stack. Software model checking technology is by now thoroughly developed for checking $\omega$-regular properties of runs for these models, when the runs are viewed as ordinary words (see [5, 8, 1]). Unfortunately, temporal logic and $\omega$-regular properties over ordinary words are inadequate for expressing a variety of properties of program executions that are useful in interprocedural program analysis and software verification. These include Hoare-like pre/post conditions on procedures, stack inspection properties, and other useful program analysis properties that go well beyond $\omega$-regular (see 2] for some examples). On the other hand, many such program analysis properties can easily be expressed when runs are viewed as nested words. Runs of Boolean Programs and RSMs can naturally be viewed as nested words once we add "summary edges" between matching calls and returns, and we can thus hope to extend model checking technology for procedural programs using richer temporal logics over nested words which remain tractable for analysis.

These considerations motivated the definition of Visibly Pushdown Languages (VPLs) [3] and the call-return temporal logic CaRet [2]. CaRet is a temporal logic over nested words 1 which extends LTL with new temporal operators that allow for navigation through a nested word both via its ordinary sequential structure, as well as its matching call-return summary structure. The standard LTL model checking algorithms for RSMs and PDSs can be extended to allow model checking of CaRet, with essentially the same complexity [2]. VPLs [3] are a richer class of languages that capture MSO-definable properties of nested words. Recently, results about VPLs have been recast in light of nested words, and in particular in terms of Nested Word Automata 44 which offer a machine acceptor for $(\omega$-)regular nested words, with all the expected closure properties.

Over ordinary words, LTL has long been considered the temporal logic of choice for program verification, not only because its temporal operators offer the right abstraction for reasoning about events over time, but because it provides a good balance between expressiveness (first-order complete), conciseness (can be exponentially more succinct compared to automata), and the complexity of model-checking (linear time in the size of the finite transition system, and PSPACE in the size of the temporal formula).

This raises the question: What is the right temporal logic for nested words?

The question obviously need not have a unique answer, particularly since nested words can arise in various application domains: for example, program verification, as we already discussed, or navigation and querying XML documents under "sequential" representation (see, e.g., [28]). However, it is reasonable to hope that any good temporal logic for nested

\footnotetext{
${ }^{1}$ Although the "nested word" terminology was not yet used in that paper.
} 
words should possess the same basic qualities that make LTL a good logic for ordinary words, namely:

(1) first-order expressive completeness: LTL has the same expressive power as first-order logic over words, and we would want the same over nested words (of course, even more expressiveness, such as full MSO, would be nice but natural temporal logics are subsumed by first order logic and any further expressiveness typically comes at a cost, even over words, of some other desirable properties);

(2) reasonable complexity for model checking and satisfiability; and

(3) nice closure properties: LTL is closed under boolean combinations including negation without any blow-up, and we would want the same for a logic over nested words.

Finally (and perhaps least easy to quantify), we want

(4) natural temporal operators with simple and intuitive semantics.

Unfortunately, the logic CaRet appears to be deficient with respect to some of these criteria: although it is easily first-order expressible, it is believed to be incomplete but proving incompleteness appears to be difficult. CaRet can express program path properties (for example, every lock operation is eventually followed by an unlock operation) and local path properties (for example, if a procedure executes a lock operation then the same procedure later executes an unlock operation before returning), but it seems incapable of expressing scope-bounded path properties (for example, every lock operation in a procedure is eventually followed by an unlock operation before the procedure returns). Such scope-bounded path properties are natural program requirements, and are expressible in the first-order logic of nested words. There is much related work in the XML community on logics for trees (see, e.g., surveys [15, 16, 29]), but they tend to have different kinds of deficiencies for our purposes: they concentrate on the hierarchical structure of the data and largely ignore its linear structure; also, they are designed for finite trees.

We introduce and study new temporal logics over nested words. The main logic we consider, Nested Word Temporal Logic (NWTL) extends LTL with both a future and past variant of the standard Until operator, which is interpreted over summary paths rather than the ordinary linear sequence of positions. A summary path is the unique shortest directed path one can take between a position in a run and some future position, if one is allowed to use both successor edges and matching call-return summary edges. We show that NWTL possesses all the desirable properties we want from a temporal logic on nested words. In particular, it is both first-order expressively complete and has good model checking complexity. Indeed we provide a tableaux construction which translates an NWTL formula into a Nested Word Automaton, enabling the standard automata theoretic approach to model checking of Boolean Programs and RSMs with complexity that is polynomial in the size the model and EXPTIME in the size of the formula (and indeed EXPTIME-complete).

We then explore some alternative temporal logics, which extend variants of CaRet with variants of unary "Within" operators proposed in [2], and we show that these extensions are also FO-complete. However, we observe that the model checking and satisfiability problems for these logics are 2EXPTIME-complete. These logics are - provably - more concise than NWTL, but we pay for conciseness with added complexity.

It follows from our proof of FO-completeness for NWTL that over nested words, every first-order formula with one free variable can be expressed using only 3 variables. More generally, we show, using EF games, that 3 variables suffice for expressing any first order formula with two or fewer free variables, similarly to the case of words [13] or finite trees 
20]. Finally, we show that a natural unary temporal logic over nested words is expressively complete for first-order logic with 2 variables, echoing a similar result known for unary temporal logic over ordinary words [9].

Related Work. VPLs and nested words were introduced in [3, 4]. The logic CaRet was defined in [2] with the goal of expressing and checking some natural non-regular program specifications. The theory of VPLs and CaRet has been recast in light of nested words in [4. Other aspects of nested words (automata characterizations, games, model-checking) were further studied in [1, 4, 2, 17]. It was also observed that nested words are closely related to a sequential, or "event-based" API for XML known as SAX [25] (as opposed to a tree-based DOM API [7]). SAX representation is very important in streaming applications, and questions related to recognizing classes of nested words by the usual word automata have been addressed in [28, 6].

While finite nested words can indeed be seen as XML documents under the SAX representation, and while much effort has been spent over the past decade on languages for tree-structured data (see, e.g., [15, 16, 29] for surveys), adapting the logics developed for tree-structured data is not as straightforward as it might seem, even though from the complexity point of view, translations between the DOM and the SAX representations are easy [27. The main problem is that most such logics rely on the tree-based representation and ignore the linear structure, making the natural navigation through nested words rather unnatural under the tree representation. Translations between DOM and SAX are easy for first-order properties, but verifying navigational properties expressed in first-order is necessarily non-elementary even for words if one wants to keep the data complexity linear [10]. On the other hand, logics for XML tend to have good model-checking properties (at least in the finite case), typically matching the complexity of LTL [11, 22]. We do employ such logics (e.g., those in [19, 20, 26]) in the proof of the expressive completeness of NWTL, first by using syntactic translations that reconcile both types of navigation, and then by combining them with a composition game argument that extends the result to the infinite case, which is not considered in the XML setting. This, however, involves a nontrivial amount of work. Furthermore, "within" operators do not have any natural analog on trees, and the proof for them is done by a direct composition argument on nested words.

Organization. Basic notations are given in Section 2. Section 3 defines temporal logics on nested words, and Section 4 presents expressive completeness results. We study modelchecking in Section 5, and in Section 6 we prove the 3-variable property and present a logic for the 2-variable fragment.

\section{Notations}

2.1. Nested Words. A matching on $\mathbb{N}$ or an interval $[1, n]$ of $\mathbb{N}$ consists of a binary relation $\mu$ and two unary relations call and ret, satisfying the following: (1) if $\mu(i, j)$ holds then $\operatorname{call}(i)$ and $\operatorname{ret}(j)$ and $i<j$; (2) if $\mu(i, j)$ and $\mu\left(i, j^{\prime}\right)$ hold then $j=j^{\prime}$ and if $\mu(i, j)$ and $\mu\left(i^{\prime}, j\right)$ hold then $i=i^{\prime}$; (3) if $i \leq j$ and $\operatorname{call}(i)$ and $\operatorname{ret}(j)$ then there exists $i \leq k \leq j$ such that either $\mu(i, k)$ or $\mu(k, j)$.

Let $\Sigma$ be a finite alphabet. A finite nested word of length $n$ over $\Sigma$ is a tuple $\bar{w}=$ $\left(w, \mu\right.$, call, ret), where $w=a_{1} \ldots a_{n} \in \Sigma^{*}$, and ( $\mu$, call, ret) is a matching on $[1, n]$. A 

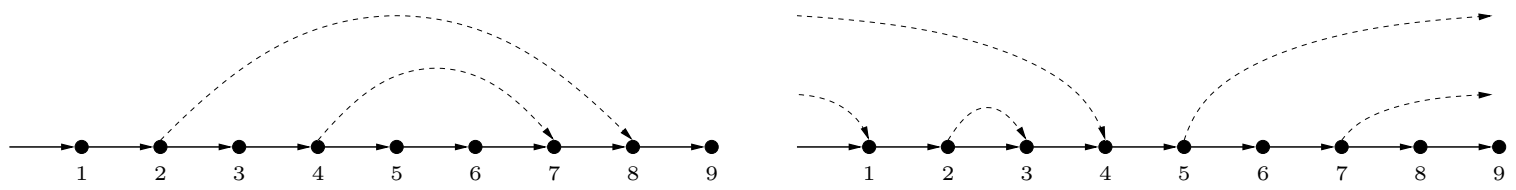

Figure 1: Sample nested words

nested $\omega$-word is a tuple $\bar{w}=(w, \mu$, call, ret $)$, where $w=a_{1} \ldots \in \Sigma^{\omega}$, and ( $\mu$, call, ret) is a matching on $\mathbb{N}$.

We say that a position $i$ in a nested word $\bar{w}$ is a call position if call $(i)$ holds; a return position if ret $(i)$ holds; and an internal position if it is neither a call nor a return. If $\mu(i, j)$ holds, we say that $i$ is the matching call of $j$, and $j$ is the matching return of $i$, and write $c(j)=i$ and $r(i)=j$. Calls without matching returns are pending calls, and returns without matching calls are pending returns (sometimes we will alternatively refer to such calls and returns as unmatched). A nested word is said to be well-matched if no calls or returns are pending. Note that for well-matched nested words, the unary predicates call and ret are uniquely specified by the relation $\mu$.

A nested word $\bar{w}=(w, \mu$, call, ret $)$ is represented as a first-order structure

$$
\left\langle U,\left(P_{a}\right)_{a \in \Sigma},<, \mu, \text { call, ret }\right\rangle,
$$

where $U$ is $\{1, \ldots, n\}$ if $w$ is a finite word of length $n$ and $\mathbb{N}$ if $\bar{w}$ is a nested $\omega$-word; < is the usual ordering, $P_{a}$ is the set of positions labeled $a$, and $(\mu, \mathrm{call}$, ret) is the matching relation. When we talk about first-order logic (FO) over nested words, we assume FO over such structures (i.e. the vocabulary is that of words plus the matching relation).

For a nested word $\bar{w}$, and two elements $i, j$ of $\bar{w}$, we denote by $\bar{w}[i, j]$ the substructure of $\bar{w}$ (i.e. a finite nested word) induced by elements $\ell$ such that $i \leq \ell \leq j$. If $j<i$ we assume that $\bar{w}[i, j]$ is the empty nested word. For nested $\omega$-words $\bar{w}$, we let $\bar{w}[i, \infty]$ denote the substructure induced by elements $l \geq i$.

When this is clear from the context, we do not distinguish references to positions in subwords $\bar{w}[i, j]$ and $\bar{w}$ itself, e.g., we shall often write $(\bar{w}[i, j], i) \models \varphi$ to mean that $\varphi$ is true at the first position of $\bar{w}[i, j]$.

Figure 1 shows two finite nested words (without the labeling with alphabet symbols). Nesting edges are drawn using dashed lines. For the first word, the relation $\mu$ is $\{(2,8),(4,7)\}$, the set call is $\{2,4\}$, and the set ret is $\{7,8\}$. For the second word, the relation $\mu$ is $\{(2,3)\}$, the set call is $\{2,5,7\}$, and the set ret is $\{1,3,4\}$.

Note that our definition allows a nesting edge from a position $i$ to its linear successor, and in that case there will be two edges from $i$ to $i+1$; this is the case for positions 2 and 3 of the second sequence. The second sequence has two pending calls and two pending returns. Pending calls are depicted by dashed outgoing edges and pending returns are depicted by dashed incoming edges. Note that all pending return positions in a nested word appear before any of the pending call positions (this is enforced by condition (3) of the definition of matchings).

2.2. Games and types. The quantifier rank (or quantifier depth) of an FO formula $\varphi$ is the depth of quantifier nesting in $\varphi$. The rank-k type of a structure $\mathfrak{M}$ over a relational vocabulary is the set $\{\varphi \mid \mathfrak{M} \models \varphi$ and the quantifier rank of $\varphi$ is $k\}$, where $\varphi$ ranges over FO sentences over the vocabulary. It is well-known that there are finitely many rank- $k$ 
types for all $k$, and for each rank- $k$ type $\tau$ there is an FO sentence $\varphi_{\tau}$ such that $\mathfrak{M} \models \varphi_{\tau}$ iff the rank- $k$ type of $\mathfrak{M}$ is $\tau$. Sometimes we associate types with formulas that define them.

Many proofs in this paper make use of Ehrenfeucht-Fraïssé (EF) games, see for example 12. This game is played on two structures, $\mathfrak{M}$ and $\mathfrak{M}^{\prime}$, over the same vocabulary, by two players, Player I and Player II. In round $i$ Player I selects a structure, say $\mathfrak{M}$, and an element $c_{i}$ in the domain of $\mathfrak{M}$; Player II responds by selecting an element $e_{i}$ in the domain of $\mathfrak{M}^{\prime}$. Player II wins in $k$ rounds, for $k \geq 0$, if $\left\{\left(c_{i}, e_{i}\right) \mid i \leq k\right\}$ defines a partial isomorphism between $\mathfrak{M}$ and $\mathfrak{M}^{\prime}$. Also, if $\bar{a}$ is an $m$-tuple in the domain of $\mathfrak{M}$ and $\bar{b}$ is an $m$-tuple in the domain of $\mathfrak{M}^{\prime}$, where $m \geq 0$, we write $(\mathfrak{M}, \bar{a}) \equiv_{k}\left(\mathfrak{M}^{\prime}, \bar{b}\right)$ whenever Player II wins in $k$ rounds no matter how Player I plays, but starting from position $(\bar{a}, \bar{b})$.

We write $\mathfrak{M} \equiv_{k} \mathfrak{M}^{\prime}$ iff $\mathfrak{M}$ and $\mathfrak{M}^{\prime}$ have the same rank- $k$ type, that is for every FO sentence $\varphi$ of quantifier rank- $k, \mathfrak{M} \models \varphi \Leftrightarrow \mathfrak{M}^{\prime} \models \varphi$. It is well-known that $\mathfrak{M} \equiv_{k} \mathfrak{M}^{\prime}$ iff Player II has a winning strategy in the $k$-round Ehrenfeucht-Fraïssè game on $\mathfrak{M}$ and $\mathfrak{M}^{\prime}$.

In the proof of Theorem 6.1, we shall also use $k$-pebble games. In such a game, Player I and Player II have access to $k$ matching pebbles each, and each round consists of Player I either removing, or placing, or replacing a pebble in one structure, and Player II replicating the move in the other structure. The correspondence given by the matching pebbles should be a partial isomorphism. If Player II can play while maintaining partial isomorphism for $m$ rounds, then the structures agree on all $\mathrm{FO}^{k}$ sentences of quantifier rank up to $m$; if Player II can play while maintaining partial isomorphism forever, then the structures agree on all $\mathrm{FO}^{k}$ sentences. ( $\mathrm{FO}^{k}$ is first-order logic where at most $k$ distinct variables may occur.)

\section{Temporal Logics over Nested Words}

We now describe our approach to temporal logics for nested words. It is similar to the approach taken by the logic CaRet [2]. Namely, we shall consider LTL-like logics that define the next/previous and until/since operators for various types of paths in nested words.

All the logics will be able to refer to propositional letters, including the base unary relations call and ret, and will be closed under all Boolean combinations. We shall write $\top$ for true and $\perp$ for false. For all the logics, we shall define the notion of satisfaction with respect to a position in a nested word: we write $(\bar{w}, i) \models \varphi$ to denote that the formula $\varphi$ is true in position $i$ of the word $\bar{w}$.

Since nested words are naturally represented as transition systems with two binary relations - the successor and the matching relation - in all our logics we introduce next operators $\bigcirc$ and $\bigcirc_{\mu}$. The semantics of those is standard: $(\bar{w}, i) \models \bigcirc \varphi$ iff $(\bar{w}, i+1) \models \varphi$, $(\bar{w}, i) \models \bigcirc_{\mu} \varphi$ iff $i$ is a call with a matching return $j$ (i.e., $\mu(i, j)$ holds) and $(\bar{w}, j) \models \varphi$. Likewise, we shall have past operators $\ominus$ and $\Theta_{\mu}$ : that is, $\Theta \varphi$ is true in position $i>1$ iff $\varphi$ is true in position $i-1$, and $\ominus_{\mu} \varphi$ is true in position $j$ if $j$ is a return position with matching call $i$ and $\varphi$ is true at $i$.

3.1. Paths in Nested Words. The until/since operators depend on what a path is. In general, there are various notions of paths through a nested word. We shall consider until/since operators for paths that are unambiguous: that is, for every pair of positions $i$ and $j$ with $i<j$, there could be at most one path between them. Then, with respect to any such given notion of a path, we have the until and since operators with the usual semantics:

- $(\bar{w}, i) \models \varphi \mathbf{U} \psi$ iff there is a position $j \geq i$ and a path $i=i_{0}<i_{1}<\ldots<i_{k}=j$ between them such that $(\bar{w}, j) \models \psi$ and $\left(\bar{w}, i_{p}\right) \models \varphi$ for every $0 \leq p<k$. 
- $(\bar{w}, i) \models \varphi \mathbf{S} \psi$ iff there is a position $j \leq i$ and a path $j=i_{0}<i_{1}<\ldots<i_{k}=i$ between them such that $(\bar{w}, j) \models \psi$ and $\left(\bar{w}, i_{p}\right) \models \varphi$ for every $0<p \leq k$.

The approach of CaRet was to introduce three types of paths, based on the linear successor (called linear paths), the call-return relation (called abstract paths), and the innermost call relation (called call paths).

To define those, we need the notions $\mathcal{C}(i)$ and $\mathcal{R}(i)$ for each position $i$ - these are the innermost call within which the current action $i$ is executed, and its corresponding return. Formally, $\mathcal{C}(i)$ is the greatest matched call position $j<i$ whose matching return is after $i$ (if such a call position exists), and $\mathcal{R}(i)$ is the least matched return position $\ell>i$ whose matching call is before $i$.

Definition 3.1 (Linear, call and abstract paths). Given two positions $i<j$, a sequence $i=i_{0}<i_{1}<\ldots<i_{k}=j$ is

- a linear path if $i_{p+1}=i_{p}+1$ for all $p<k$;

- a call path if $i_{p}=\mathcal{C}\left(i_{p+1}\right)$ for all $p<k$;

- an abstract path if

$$
i_{p+1}=\left\{\begin{array}{l}
r\left(i_{p}\right) \text { if } i_{p} \text { is a matched call } \\
i_{p}+1 \text { if } i_{p} \text { is not a call and } i_{p}+1 \text { is not a matched return. }
\end{array}\right.
$$

We shall denote until/since operators corresponding to these paths by $\mathbf{U} / \mathbf{S}$ for linear paths, $\mathbf{U}^{c} / \mathbf{S}^{c}$ for call paths, and $\mathbf{U}^{a} / \mathbf{S}^{a}$ for abstract paths.

Our logics will have some of the next/previous and until/since operators. Some examples are:

- When we restrict ourselves to the purely linear fragment, our operators are $\bigcirc$ and $\ominus$, and $\mathbf{U}$ and $\mathbf{S}$, i.e., precisely LTL (with past operators).

- The logic CaRet 2] has the following operators: the next operators $\bigcirc$ and $\bigcirc_{\mu}$; the linear and abstract untils (i.e., $\mathbf{U}$ and $\mathbf{U}^{a}$ ), the call since (i.e., $\mathbf{S}^{c}$ ) and a previous operator $\Theta_{c}$, defined by: $(\bar{w}, i) \models \Theta_{c} \varphi$ iff $\mathcal{C}(i)$ is defined and $(\bar{w}, \mathcal{C}(i)) \models \varphi$.

Another notion of a path combines both the linear and the nesting structure. It is the shortest directed path between two positions $i$ and $j$. Unlike an abstract path, it decides when to skip a call based on position $j$. Basically, a summary path from $i$ to $j$ moves along successor edges until it finds a call position $k$. If $k$ has a matching return $\ell$ such that $j$ appears after $\ell$, then the summary path skips the entire call from $k$ to $\ell$ and continues from $\ell$; otherwise the path continues as a successor path. Note that every abstract path is a summary path, but there are summary paths that are not abstract paths.

Definition 3.2. A summary path between $i<j$ in a nested word $\bar{w}$ is a sequence $i=i_{0}<$ $i_{1}<\ldots<i_{k}=j$ such that for all $p<k$,

$$
i_{p+1}=\left\{\begin{array}{l}
r\left(i_{p}\right) \text { if } i_{p} \text { is a matched call and } j \geq r\left(i_{p}\right) \\
i_{p}+1 \text { otherwise }
\end{array}\right.
$$

The corresponding until/since operators are denoted by $\mathbf{U}^{\sigma}$ and $\mathbf{S}^{\sigma}$.

We will also consider two special kinds of summary paths: summary-down paths are allowed to use only call edges (from a call position, $i$ to $i+1$ where $i+1$ is not a return), nesting edges (from a call to its matching return), and internal edges (from some $i$ to $i+1$ where $i$ is not a call and $i+1$ is not a return), and summary-up paths are allowed to use 
only return edges (from a position preceding a return to the return), nesting edges and internal edges. (In other words, summary-down paths are summary paths with no return edges and summary-up paths are summary paths with no call edges.)

We will use $\mathbf{U}^{\sigma \downarrow}$ and $\mathbf{U}^{\sigma \uparrow}$ to denote the corresponding until operators. A general summary path is a concatenation of a summary-up path and summary-down path: $\varphi \mathbf{U}^{\sigma} \psi$ is equivalent to $\varphi \mathbf{U}^{\sigma \uparrow}\left(\varphi \mathbf{U}^{\sigma \downarrow} \psi\right)$.

We will also study the expressiveness of various until modalities when the logic is extended with the within operator, which allows restriction to a subword. If $\varphi$ is a formula, then $\mathcal{W} \varphi$ is a formula, and $(\bar{w}, i) \models \mathcal{W} \varphi$ iff $i$ is a call, and $(\bar{w}[i, j], i) \models \varphi$, where $j=r(i)$ if $i$ is a matched call, $j=|\bar{w}|$ if $i$ is an unmatched call and $\bar{w}$ is finite, and $j=\infty$ otherwise. In other words, $\mathcal{W} \varphi$ evaluates $\varphi$ on a subword restricted to a single procedure.

To understand the various notions of paths in a nested word, let us consider the left word shown in Figure 1 again. An abstract path uses internal and nesting edges; for example, $\langle 1,2,8,9\rangle$ and $\langle 3,4,7\rangle$ are abstract paths. Summary-down paths, in addition, can use call edges; for example, $\langle 1,2,3,4,7\rangle$ is a summary-down (but not an abstract) path. Summary-up paths can use internal and nesting edges, and can also go along return edges; for example, $\langle 3,4,7,8,9\rangle$ is a summary-up path. A summary path is a summary-up path followed by a summary-down path; for example, $\langle 3,4,5,6,7\rangle$ in the right word of Figure 1 is a summary path (which also happens to be a linear path). Every two positions have a unique summary path connecting them, and this is the "shortest" path in the underlying graph between these positions.

3.2. Specifying Requirements. We now discuss how the various operators can be used for specifying requirements for sequential structured programs. In the classical linear-time semantics of programs, an execution of a program is modeled as a word over program states. In the nested-word semantics, this linear structure is augmented with nesting edges from entries to exits of program blocks. The main benefit is that using nesting edges one can skip procedure calls entirely, and continue to trace a local path through the calling procedure. A program is now viewed as a generator of nested words, and requirements are written using temporal logics over nested words.

Suppose we want to express the requirement that, along a global program execution, every write to a variable is followed by a read before the variable is written again. If $w r$ and $r d$ denote the atomic propositions that capture write and read operations, respectively, then the requirement is expressed by the until formula over linear paths,

$$
[w r \rightarrow(\neg w r) \mathbf{U} r d]
$$

Here, $\square$ is defined in the usual manner from the linear until: $\square \varphi$ stands for $\neg(\top \mathbf{U} \neg \varphi)$. This property is clearly already expressible in LTL and does not use nesting edges at all.

Now let us review some of the properties expressible in the nested call-return logic CaRet of [2], but not expressible in LTL. In the classical verification formalisms such as Hoare logic, correctness of procedures is expressed using pre and post conditions. Partial correctness of a procedure $A$ specifies that if the pre-condition $p$ holds when the procedure $A$ is invoked, then if the procedure terminates, the post-condition $q$ is satisfied upon return. Total correctness, in addition, requires the procedure to terminate. Assume that all calls to the procedure $A$ are characterized by the proposition $p_{A}$. Then, the requirement

$$
\left[\left(\operatorname{call} \wedge p \wedge p_{A}\right) \rightarrow \bigcirc_{\mu} q\right]
$$


expresses the total correctness, while

$$
\square\left[\left(\bigcirc_{\mu} \top \wedge p \wedge p_{A}\right) \rightarrow \bigcirc_{\mu} q\right]
$$

expresses the partial correctness. Both these specifications crucially rely upon the abstractnext operator.

The abstract path starting at a position inside a procedure $A$ is obtained by successive applications of internal and nesting edges, and skips over invocations of other procedures called from $A$. Using the abstract versions of temporal operators, we can specify properties of such abstract paths. For example, suppose we want to specify that if a procedure writes to a variable, then it (that is, the same invocation of the same procedure) will later read it and do so before writing to it again. The requirement is expressed by the until formula over abstract paths

$$
\square\left[w r \rightarrow \bigcirc(\neg w r) \mathbf{U}^{a} r d\right]
$$

The call since-path starting at a position inside a procedure $A$ is obtained by successively jumping to the innermost call positions, and encodes the active stack at that position. Stack inspection can specify a variety of security properties. For instance, the requirement that a procedure $A$ should be invoked only within the context of a procedure $B$, with no intervening call to an overriding module $C$, is expressed by the formula

$$
\square\left[\operatorname{call} \wedge p_{A} \rightarrow\left(\neg p_{C}\right) \mathbf{S}^{c} p_{B}\right] .
$$

Finally, we turn to scope-bounded linear-path properties. For a procedure, the corresponding scope-bounded linear path is the linear path (that is, the path obtained by following linear edges) from its call to it return. That is, a scope-bounded path corresponding to a procedure $P$ includes the executions of the procedures (transitively) called by $P$, but terminates when the current invocation of $P$ returns. Properties about scope-bounded paths are useful in asserting contracts for modules.

Suppose we want to assert that a procedure $A$, and the procedures it calls, do not write to a variable before it returns. This is an invariant of the scope-bounded path, and is captured by the formula:

$$
\square\left[\left(\operatorname{call} \wedge p_{A}\right) \rightarrow \mathcal{W}(\square \neg w r)\right]
$$

Recall that the within operator $\mathcal{W}$ restricts the evaluation of a formula to a single procedure call. The same requirement can also be captured using summary paths. It is even easier to state it using summary-down paths:

$$
\square\left[\left(\operatorname{call} \wedge p_{A}\right) \rightarrow \neg\left(\top \mathbf{U}^{\sigma \downarrow} w r\right)\right]
$$

Suppose we want to specify the requirement that if a procedure writes to a variable then it is read along the scope-bounded path before being written again. We can use the within modality to express this property:

$$
[\operatorname{call} \rightarrow \mathcal{W} \square(w r \rightarrow \bigcirc(\neg w r) \mathbf{U} r d)]
$$

This requirement can also be alternatively specified using summary-down paths as follows:

$$
\square\left[w r \rightarrow \bigcirc\left(\neg w r \wedge\left(\text { ret } \rightarrow \Theta_{\mu} \neg \top \mathbf{U}^{\sigma \downarrow} w r\right)\right) \mathbf{U}^{\sigma \downarrow} r d\right]
$$

The formula says that from every write operation, there is a read operation along some summary-down path (and thus, within the same scope) such that along the path, there is no write, and if the path uses a summary edge, then the enclosed subword also does not contain a write. 
It is easy to see that the above requirements concerning scope-bounded paths are specifiable in the first-order logic of nested words. It is conjectured that they are not specifiable in CaRet.

\section{Expressive Completeness}

In this section, we study logics that are expressively complete for FO, i.e. temporal logics that have exactly the same power as FO formulas with one free variable over finite and infinite nested words. In other words, for every formula $\varphi$ of an expressively complete temporal logic there is an FO formula $\varphi^{\prime}(x)$ such that $(\bar{w}, i) \models \varphi$ iff $\bar{w} \models \varphi^{\prime}(i)$ for every nested word $\bar{w}$ and position $i$ in it, and conversely, for every FO formula $\psi(x)$ there is a temporal formula $\psi^{\prime}$ such that $\bar{w} \models \psi(i)$ iff $(\bar{w}, i) \models \psi^{\prime}$.

Our starting point is a logic NWTL (nested-word temporal logic) based on summary paths introduced in the previous section. We show that this logic is expressively complete for FO, and of course remains expressively complete with the addition of other first-order expressible operators which may be useful for verification of properties of procedural programs. When we provide upper bounds on the complexity of model checking for NWTL, we shall in fact show that the upper bounds hold with respect to an extension, $\mathrm{NWTL}^{+}$, which includes a number of additional operators.

We then look at logics close to those in the verification literature, i.e., with operators such as call and abstract until and since, and ask what needs to be added to them to get expressive completeness. We confirm a conjecture of [2] that a within operator is sufficient. Such an operator evaluates a formula on a nested subword. We then discuss the role of this within operator. We show that, if added to NWTL, it does not increase expressiveness, but makes the logic exponentially more succinct.

4.1. Expressive completeness and NWTL. The logic NWTL (nested words temporal logic) has next and previous operators, as well as until and since with respect to summary paths. That is, its formulas are given by:

$$
\begin{aligned}
\varphi, \varphi^{\prime}:= & \top|a| \text { call } \mid \text { ret }|\neg \varphi| \varphi \vee \varphi^{\prime} \mid \\
& \bigcirc_{\varphi}\left|\bigcirc_{\mu} \varphi\right| \Theta^{\prime}\left|\Theta_{\mu} \varphi\right| \\
& \varphi \mathbf{U}^{\sigma} \varphi^{\prime} \mid \varphi \mathbf{S}^{\sigma} \varphi^{\prime}
\end{aligned}
$$

where $a$ ranges over $\Sigma$. We use abbreviations int for $\neg$ call $\wedge \neg$ ret (true in an internal position). Note that in the absence of pending calls and returns, call and ret are definable as $\bigcirc_{\mu} \top$ and $\Theta_{\mu} \top$, respectively.

Theorem 4.1. NWTL = FO over both finite and infinite nested words.

Proof. We start with the easy direction NWTL $\subseteq$ FO.

Lemma 4.2. For every NWTL formula $\varphi$, there exists an FO formula $\alpha_{\varphi}(x)$ that uses at most three variables $x, y, z$ such that for every nested word $\bar{w}$ (finite or infinite), and every position, $i$ in $\bar{w}$, we have $(\bar{w}, i) \models \varphi$ iff $\bar{w} \models \alpha_{\varphi}(i)$.

Proof of Lemma 4.2. The proof is by induction on the formulas and very simple for all the cases except $\mathbf{U}^{\sigma}$ and $\mathbf{S}^{\sigma}$ : for example,

$$
\alpha_{\bigcirc_{\mu} \varphi}(x)=\exists y\left(\mu(x, y) \wedge \exists x\left(x=y \wedge \alpha_{\varphi}(x)\right)\right) .
$$


For translating $\mathbf{U}^{\sigma}$, we need a few auxiliary formulas. Our first goal is to define a formula $\gamma_{r}(x, z)$ saying that $x$ is $\mathcal{R}(z)$, i.e. the return of the innermost call within which $z$ is executed. For that, we start with $\delta(y, z)=z<y \wedge \operatorname{ret}(y) \wedge \exists x(\mu(x, y) \wedge x<z)$ saying that $y$ is a return that is preceded by $z$ and whose matching call precedes $z$, that is, $y$ is a candidate for $\mathcal{R}(z)$. Then the formula $\gamma_{r}(x, z)$ is given by

$$
\exists y(y=x \wedge \delta(y, z)) \wedge \forall y(\delta(y, z) \rightarrow y \geq x) .
$$

Likewise, we define $\gamma_{c}(y, z)$ stating that that $y$ equals $\mathcal{C}(z)$, that is, the innermost call within which $z$ is executed. Now define

$$
\chi_{1}(y, z)=\exists x\left(\gamma_{r}(x, z) \wedge x \leq y\right), \quad \chi_{2}(x, z)=\exists y\left(\gamma_{c}(y, z) \wedge y \geq x\right)
$$

and $\chi(x, y, z)$ as $\chi_{1}(y, z) \wedge \chi_{2}(x, z)$. Then this formula says that the summary path from $x$ to $y$ does not pass through $z$, assuming $x<z<y$. With this, $\alpha_{\varphi \mathbf{U}^{\sigma} \psi}(x)$ is given by

$$
\begin{aligned}
& \alpha_{\psi}(x) \vee \exists y\left(y>x \wedge \alpha_{\varphi}(x) \wedge \exists x\right.\left(x=y \wedge \alpha_{\psi}(x)\right) \wedge \\
&\left.\forall z\left((x<z<y \wedge \neg \chi(x, y, z)) \rightarrow \exists x\left(x=z \wedge \alpha_{\varphi}(x)\right)\right)\right)
\end{aligned}
$$

The proof for $\varphi \mathbf{S}^{\sigma} \psi$ is similar. This concludes the proof of the lemma.

In the proof of the other direction, FO $\subseteq$ NWTL, we shall use a tree representation of nested words. The translation is essentially the same as in [4. For each nested word $\bar{w}$ we have a binary tree $T_{\bar{w}}$ (i.e., its nodes are elements of $\{0,1\}^{*}$ ) and a function $\iota_{\mathrm{w}-\mathrm{t}}: \bar{w} \rightarrow T_{\bar{w}}$ that maps each position of $\bar{w}$ to a node of $T_{\bar{w}}$ as follows:

- the first position of $\bar{w}$ is mapped into the root of $T_{\bar{w}}$;

- if $s=\iota_{\mathrm{w}-\mathrm{t}}(i)$ then:

(1) if $i$ is an internal, or an unmatched call, or a matched call whose return is the last position of $\bar{w}$, or an unmatched return, and $i$ is not the last position of $\bar{w}$, then $s$ has only child $s \cdot 0$ and $\iota_{\mathrm{w}-\mathrm{t}}(i+1)=s \cdot 0$;

(2) if $i$ is a matched call whose return is not the last position in $\bar{w}$, then $s$ has both children $s \cdot 0$ and $s \cdot 1$ and $\iota_{\mathrm{w}-\mathrm{t}}(r(i)+1)=s \cdot 0$, and $\iota_{\mathrm{w}-\mathrm{t}}(i+1)=s \cdot 1$.

(3) if $i$ is a matched return, then $s$ has no children.

The $\Sigma$-labels of $i$ and $\iota_{\mathrm{w}-\mathrm{t}}(i)$ are the same. If $i$ was a pending call, we label $\iota_{\mathrm{w}-\mathrm{t}}(i)$ with pcall, and if $i$ was a pending return, we label $\iota_{\mathrm{w}-\mathrm{t}}(i)$ with pret.

Note that $\iota_{\mathrm{w}-\mathrm{t}}$ is a bijection, and that labels pcall and pret may only occur on the leftmost branch of $T_{\bar{w}}$. An example of a nested word and its translation are given in Fig. 2,

To relate paths in nested words and paths in their tree translations, we introduce the notions of semi-strict and strict paths. Intuitively, a semi-strict path in a nested word corresponds to a path on its tree translation that, in addition to following tree edges, can jump from a node with no children to its successor in the depth-first traversal of the tree (where depth-first starts with the right subtree and then moves to the left subtree). A strict path is just a path that follows tree edges. These are both slight modifications of summary paths.

More precisely, a semi-strict path between positions $i$ and $j$, with $i<j$, in a nested word $\bar{w}$, is a sequence $i=i_{0}<i_{1}<\cdots<i_{k}=j$ such that

$$
i_{p+1}=\left\{\begin{array}{l}
r\left(i_{p}\right)+1 \text { if } i_{p} \text { is a matched call and } j>r\left(i_{p}\right) \\
i_{p}+1 \text { otherwise }
\end{array}\right.
$$



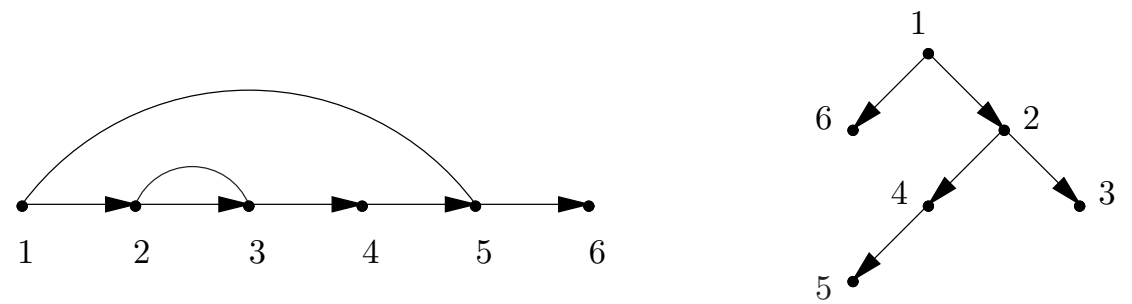

Figure 2: A nested word and its tree translation

That is, when skipping a call, instead of jumping to the matching return position, a semistrict path will jump to its successor.

A strict path is a semi-strict path $i=i_{0}<i_{1}<i_{2}<\cdots<i_{k}=j$ in which no $i_{p}$ with $p<k$ is a matched return position. In other words, a strict path stops if it reaches a matched return position. In particular there may be positions $i<j$ in a nested word such that no strict path exists between them.

For example, in Fig. 2, $\langle 2,4,5,6\rangle$ is a semi-strict path. Although $\langle 2,4,5,6\rangle$ is not a path in the tree (we jump from 5 to 6 ), this is allowed under the definition of semi-strict paths. Strict paths are exactly the paths on the tree; for example, $\langle 1,2,4,5\rangle$ is such a path.

The until/since operators for semi-strict paths and strict paths will be denoted by $\mathbf{U}_{s s}^{\sigma} / \mathbf{S}_{s s}^{\sigma}$ and $\mathbf{U}_{s}^{\sigma} / \mathbf{S}_{s}^{\sigma}$, respectively. Versions of NWTL in which $\mathbf{U}^{\sigma} / \mathbf{S}^{\sigma}$ are replaced by $\mathbf{U}_{s s}^{\sigma} / \mathbf{S}_{s s}^{\sigma}\left(\mathbf{U}_{s}^{\sigma} / \mathbf{S}_{s}^{\sigma}\right)$ will be denoted by NWTL ${ }^{s s}\left(\mathrm{NWTL}^{s}\right)$.

We will use mret for ret $\wedge \Theta_{\mu} \top$, and mcall for call $\wedge \bigcirc_{\mu} \top$, to capture matching return and call positions, respectively.

The proof is based on two lemmas.

Lemma 4.3. $\mathrm{NWTL}^{s} \subseteq \mathrm{NWTL}^{s s} \subseteq$ NWTL.

Lemma 4.4. FO $\subseteq \mathrm{NWTL}^{s}$.

This of course implies the theorem: NWTL $\subseteq$ FO $\subseteq \mathrm{NWTL}^{s} \subseteq \mathrm{NWTL}^{s s} \subseteq \mathrm{NWTL}$. Note that as a corollary we also obtain $\mathrm{NWTL}^{s}=\mathrm{NWTL}^{s s}=\mathrm{FO}$.

Proof of Lemma 4.3. For translating an $\mathrm{NWTL}^{s}$ formula $\varphi$ into an equivalent formula $\alpha_{\varphi}$ of NWTL ${ }^{s s}$ we need to express $\psi \mathbf{U}_{s}^{\sigma} \theta$ with $\mathbf{U}_{s s}^{\sigma}$, which is simply $\left(\alpha_{\psi} \wedge \neg\right.$ mret $) \mathbf{U}_{s s}^{\sigma} \alpha_{\theta}$, and likewise for the since operators. For translating each NWTL ${ }^{s s}$ formula $\varphi$ into an equivalent NWTL formula $\beta_{\varphi}$, again we need to consider only the case of until/since operators. The 
formula $\psi \mathbf{U}_{s s}^{\sigma} \theta$ is translated into

$$
\begin{aligned}
\beta_{\theta} \vee & \left(\beta _ { \psi } \wedge \left(\left(\left(\beta_{\psi} \vee \text { ret }\right) \wedge\left(\neg \text { mcall } \rightarrow \bigcirc \beta_{\psi}\right) \wedge\left(\operatorname{mcall} \rightarrow\left(\bigcirc_{\mu} \bigcirc \beta_{\psi} \vee \bigcirc_{\mu} \bigcirc \beta_{\theta}\right)\right)\right) \mathbf{U}^{\sigma}\right.\right. \\
& \left.\left.\left(\left(\beta_{\psi} \vee \text { ret }\right) \wedge\left(\neg \text { mcall } \rightarrow \bigcirc \beta_{\theta}\right) \wedge\left(\operatorname{mcall} \rightarrow\left(\bigcirc \beta_{\theta} \vee \bigcirc_{\mu} \bigcirc \beta_{\theta} \vee \bigcirc(\neg \text { ret } \wedge \gamma)\right)\right)\right)\right)\right),
\end{aligned}
$$

where $\gamma$ is a formula defined as follows:

$$
\begin{aligned}
&\left(\left(\beta_{\psi} \vee \text { ret }\right) \wedge\left(\neg \text { mcall } \rightarrow \bigcirc \beta_{\psi}\right) \wedge(\operatorname{mcall}\right.\left.\left.\rightarrow\left(\bigcirc \beta_{\psi} \vee \bigcirc_{\mu} \bigcirc \beta_{\psi}\right)\right) \wedge(\text { ret } \rightarrow \text { call })\right) \mathbf{U}^{\sigma} \\
&\left(\left(\beta_{\psi} \vee \text { ret }\right) \wedge\left(\neg \text { mcall } \rightarrow \bigcirc \beta_{\theta}\right) \wedge\left(\text { mcall } \rightarrow\left(\bigcirc \beta_{\theta} \vee \bigcirc_{\mu} \bigcirc \beta_{\theta}\right)\right)\right)
\end{aligned}
$$

The idea is that we split a semi-strict path into a semi-strict up path (where call edges are excluded) followed by a semi-strict down path (where return edges are excluded). The first Until in (4.1) captures the semi-strict up path and the second Until in $\gamma$ captures the semi-strict down path. The translation for $\mathbf{S}_{s s}^{\sigma}$ is similar.

The proof that the translation is correct is a rather detailed case analysis which we have relegated to the appendix.

Proof of Lemma 4.4. We start with the finite case, and then show how the inclusion extends to nested $\omega$-words.

As a tool we shall need a slight modification of a result from [26, 19] providing an expressively complete temporal logic for trees with at most binary branching. We consider binary trees whose domain $D$ is a prefix-closed subset of $\{0,1\}^{*}$, and we impose a condition that if $s \cdot 1 \in D$ then $s \cdot 0 \in D$. When we refer to FO on trees, we assume they have two successor relations $S_{0}, S_{1}$ and the descendant relation $\preceq$ (which is just the prefix relation on strings) plus the labeling predicates, which include two new labels pcall and pret (for pending calls and returns). Each node can be labeled by either a letter from $\Sigma$, or by a letter from $\Sigma$ and pcall, or by a letter from $\Sigma$ and pret (i.e. labels pcall and pret need not be disjoint from other labels).

We also consider the following logic $\mathrm{TL}^{\text {tree }}$

$$
\begin{aligned}
\varphi:= & a|\varphi \vee \varphi| \neg \varphi \mid \\
& \bigcirc_{\downarrow}\left|\bigcirc_{\uparrow}\right| \bigcirc_{\rightarrow}\left|\bigcirc_{\leftarrow}\right| \\
& \varphi \mathbf{U}_{\downarrow} \mid \varphi \mathbf{S}_{\downarrow} \varphi
\end{aligned}
$$

where $a$ ranges over $\Sigma \cup\{$ pcall, pret $\}$, with the following semantics:

- $(T, s) \models \bigcirc_{\downarrow} \varphi$ iff $(T, s \cdot i) \models \varphi$ for some $i \in\{0,1\}$;

- $(T, s \cdot i) \models O_{\uparrow} \varphi$ iff $(T, s) \models \varphi$ (where $i$ is either 0 or 1$)$;

- $(T, s \cdot 0) \models \bigcirc \rightarrow \varphi$ iff $(T, s \cdot 1) \models \varphi$;

- $(T, s \cdot 1) \models \bigcirc_{\leftarrow \varphi}$ iff $(T, s \cdot 0) \models \varphi$;

- $(T, s) \models \varphi \mathbf{U}_{\downarrow} \psi$ iff there exists $s^{\prime}$ such that $s \preceq s^{\prime},\left(T, s^{\prime}\right) \models \psi$, and $\left(T, s^{\prime \prime}\right) \models \varphi$ for all $s^{\prime \prime}$ such that $s \preceq s^{\prime \prime} \prec s^{\prime}$;

- $(T, s) \models \varphi \mathbf{S}_{\downarrow} \psi$ iff there exists $s^{\prime}$ such that $s^{\prime} \preceq s,\left(T, s^{\prime}\right) \models \psi$, and $\left(T, s^{\prime \prime}\right) \models \varphi$ for all $s^{\prime \prime}$ such that $s^{\prime} \prec s^{\prime \prime} \preceq s$.

Lemma 4.5. (see [19]) For unary queries over finite binary trees, $\mathrm{TL}^{\text {tree }}=\mathrm{FO}$.

This lemma is an immediate corollary of expressive completeness of logic $\mathcal{X}_{\text {until }}$ from [19] on ordered unranked trees, as for a fixed number of siblings, the until and since operators 
can be expressed in terms of the next and previous operators. The result of [19] applies to arbitrary alphabets, and thus in particular to our labeling that may use pcall and pret.

The following is immediate by using the tree representation of nested words and a straightforward translation of formulae.

Claim 4.6. For every FO formula $\varphi(x)$ over nested words there is an FO formula $\varphi^{\prime}(x)$ over trees such that for every nested word $\bar{w}$ and a position $i$ in it, we have $\bar{w} \models \varphi(i)$ iff $T_{\bar{w}} \models \varphi^{\prime}\left(\iota_{\mathrm{w}-\mathrm{t}}(i)\right)$.

In fact the converse, that FO over trees $\mathfrak{T}_{\bar{w}}$ can be translated into FO over nested words, is true too, but we do not need it in this proof.

Since FO $=\mathrm{TL}^{\text {tree }}$ by Lemma 4.5, all that remains to prove is the following claim.

Claim 4.7. For every $\mathrm{TL}^{\text {tree }}$ formula $\varphi$, there exists an $\mathrm{NWTL}^{s}$ formula $\varphi^{\circ}$ such that for every nested word $\bar{w}$ and every position $i$ in it, we have

$$
(\bar{w}, i) \models \varphi^{\circ} \quad \Leftrightarrow \quad\left(T_{\bar{w}}, \iota_{\mathrm{w}-\mathrm{t}}(i)\right) \models \varphi .
$$

This is now done by induction, omitting the obvious cases of propositional letters and Boolean connectives. We note that a path down the tree from $\iota_{\mathrm{w}-\mathrm{t}}(i)$ to $\iota_{\mathrm{w}-\mathrm{t}}(j)$ corresponds precisely to the strict path from $i$ to $j$ (that is, if such a strict path is $i=i_{0}, i_{1}, \ldots, i_{k}=j$, then $\iota_{\mathrm{w}-\mathrm{t}}\left(i_{0}\right), \iota_{\mathrm{w}-\mathrm{t}}\left(i_{1}\right), \ldots, \iota_{\mathrm{w}-\mathrm{t}}\left(i_{k}\right)$ is the path from $\iota_{\mathrm{w}-\mathrm{t}}(i)$ to $\iota_{\mathrm{w}-\mathrm{t}}(j)$ in $\left.T_{\bar{w}}\right)$. Hence, the translations of until and since operators are:

$$
\left(\varphi \mathbf{U}_{\downarrow} \psi\right)^{\circ}=\varphi^{\circ} \mathbf{U}_{s}^{\sigma} \psi^{\circ}, \quad\left(\varphi \mathbf{S}_{\downarrow} \psi\right)^{\circ}=\varphi^{\circ} \mathbf{S}_{s}^{\sigma} \psi^{\circ} .
$$

For translating next and previous operators, and pending calls/returns, define:

$$
\begin{aligned}
\text { mcall } & \equiv \bigcirc_{\mu} \top \text { (true in a matched call position); } \\
\text { mret } & \equiv \Theta_{\mu} \top \text { (true in a matched return position). }
\end{aligned}
$$

Then the rest of the translation is as follows:

$$
\begin{aligned}
& \operatorname{pcall}^{\circ} \equiv \operatorname{call} \wedge \neg \mathrm{mcall} \\
& \text { pret }^{\circ} \equiv \operatorname{ret} \wedge \neg \text { mret } \\
& \left(\bigcirc_{\downarrow} \varphi\right)^{\circ} \equiv \neg \operatorname{mret} \wedge\left(\bigcirc \varphi^{\circ} \vee\left(\operatorname{call} \wedge \bigcirc_{\mu} \bigcirc \varphi^{\circ}\right)\right) \\
& \left(\bigcirc_{\uparrow} \varphi\right)^{\circ} \equiv\left(\ominus \operatorname{ret} \wedge \Theta_{\mu} \varphi^{\circ}\right) \vee\left(\Theta \neg \text { mret } \wedge \ominus^{\circ}\right)
\end{aligned}
$$

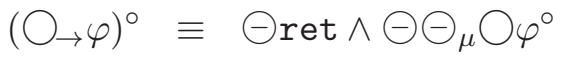

$$
\begin{aligned}
& \left(\bigcirc_{\leftarrow \varphi}\right)^{\circ} \equiv \Theta \operatorname{call} \wedge \Theta_{\mu} \bigcirc \varphi^{\circ}
\end{aligned}
$$

Now with the proof completed for finite nested words, we extend it to the case of nested $\omega$ words. Note that Claim 4.6 continues to hold, and Claim 4.7provides a syntactic translation that applies to both finite and infinite nested words, and thus it suffices to prove an analog of Lemma 4.5 for trees of the form $T_{\bar{w}}$, where $\bar{w}$ ranges over nested $\omega$-words.

If $\bar{w}$ is a nested $\omega$-word, then $T_{\bar{w}}$ has exactly one infinite branch, which consists precisely of all nodes of the form $\iota_{\mathrm{w}-\mathrm{t}}(i)$ where $i$ is an outer position, i.e., not inside any (matched) call. We say that $i$ is inside a call if there exists a call $j$ with a matching return $k$ such that $j<i \leq k$. If $i$ is an outer position, then we shall call $\iota_{\mathrm{w}-\mathrm{t}}(i)$ an outer node in the tree $T_{\bar{w}}$ as well.

If $i$ is an outer position which is not a matched call, then $i+1$ is also an outer position and $\iota_{\mathrm{w}-\mathrm{t}}(i+1)$ is the left successor of $\iota_{\mathrm{w}-\mathrm{t}}(i)$. If $i$ is an outer position and a call with $j>i$ 
being its matching return, then the left successor of $\iota_{\mathrm{w}-\mathrm{t}}(i)$ on the infinite path is $\iota_{\mathrm{w}-\mathrm{t}}(j+1)$. Furthermore, the subtree $t^{\bar{w}}(i)$, which has $\iota_{\mathrm{w}-\mathrm{t}}(i)$ as the root, plus its right child, and all the descendants of the right child, is finite and isomorphic to $T_{\bar{w}[i, j]}$ (note that $\bar{w}[i, j]$ has no pending calls/returns). If $i$ is an outer position other than a matched call, we let $t^{\bar{w}}(i)$ be a single node tree labeled with $i$ 's label in $\bar{w}$.

Let $\bar{w}$ now be a nested $\omega$-word. For each outer position $i$ we let $\tau_{m}^{\bar{w}}(i)$ be the rank- $m$ type of $t^{\bar{w}}(i)$. If $i$ is not a matched call, such a type is completely described by $i$ 's label (which consists of a label in $\Sigma$ and potentially pcall or pret).

If $j$ is not an outer position, and $i$ is an outer position such that $i<j \leq k$, where $k$ is the matching return of $i$, then $\tau_{m}^{\bar{w}}(j)$ is the rank- $m$ type of $\left(T_{\bar{w}}[i, k], \iota_{\mathrm{w}-\mathrm{t}}(j)\right)$ (i.e., the type of $T_{\bar{w}}[i, k]$ with a distinguished node corresponding to $j$ ).

Next, for a nested $\omega$-word $\bar{w}$, let $s$ be a node in $T_{\bar{w}}$ such that $s=\iota_{\mathrm{w}-\mathrm{t}}(i)$. Let $i_{1}, i_{2}, \ldots$ enumerate all the outer positions of $\bar{w}$, and assume that $i_{p}$ is such that $i_{p} \leq i<i_{p+1}$ - that is, $\iota_{\mathrm{w}-\mathrm{t}}(i)$ is a node in the subtree $t^{\bar{w}}\left(i_{p}\right)$. We now define a finite word $s_{m}^{\overleftarrow{w}}(\bar{w}, s)$ of length $p-1$ such that its positions $1, \ldots, p-1$ are labeled $\tau_{m}^{\bar{w}}\left(i_{1}\right), \ldots, \tau_{m}^{\bar{w}}\left(i_{p-1}\right)$, and an $\omega$-word $s_{m}(\bar{w}, s)$ such that its positions $1,2, \ldots$ are labeled by $\tau_{m}^{\bar{w}}\left(i_{p+1}\right), \tau_{m}^{\bar{w}}\left(i_{p+2}\right), \ldots$ Next we show:

Claim 4.8. Let $\bar{w}, \bar{w}^{\prime}$ be two nested $\omega$-words, and $s=\iota_{\mathrm{w}-\mathrm{t}}(i), s^{\prime}=\iota_{\mathrm{w}-\mathrm{t}}\left(i^{\prime}\right)$ two nodes in $T_{\bar{w}}$ and $T_{\bar{w}^{\prime}}$ such that:

(a) $s_{m}^{\leftarrow}(\bar{w}, s) \equiv_{m} s_{m}^{\overleftarrow{m}}\left(\bar{w}^{\prime}, s^{\prime}\right)$

(b) $s_{m}(\bar{w}, s) \equiv_{m} s_{m}\left(\bar{w}^{\prime}, s^{\prime}\right)$;

(c) $\tau_{m}^{\bar{w}}(i)=\tau_{m}^{\bar{w}^{\prime}}\left(i^{\prime}\right)$.

Then $\left(T_{\bar{w}}, s\right) \equiv_{m}\left(T_{\bar{w}^{\prime}}, s^{\prime}\right)$.

Proof. A standard composition argument shows that Player II wins. If $i_{1}, i_{2}, \ldots$ enumerate outer positions in $\bar{w}$ and $i_{p} \leq i<i_{p+1}$, then a move by Player I, say, in $T_{\bar{w}}$, occurs either in $t^{\bar{w}}(j)$ with $j<i$, or in $t^{\bar{w}}(i)$, or in $t^{\bar{w}}(j)$ with $j>i$. Player II then selects $j^{\prime}$ so that the response is in $t^{\bar{w}^{\prime}}\left(j^{\prime}\right)$ according to his winning strategy in games either (a) or (b) (if $j$ is in $t^{\bar{w}}(i)$, then $j^{\prime}$ is in $\left.\tau_{m}^{\bar{w}^{\prime}}\left(i^{\prime}\right)\right)$, and then, since the rank- $m$ types of $t^{\bar{w}}(j)$ and the chosen $t^{\bar{w}^{\prime}}\left(j^{\prime}\right)$ are the same, selects the actual response according to the winning strategy $t^{\bar{w}}(j) \equiv_{m} t^{\bar{w}^{\prime}}\left(j^{\prime}\right)$.

Next we show how Claim 4.8 proves that FO is expressible in $\mathrm{TL}^{\text {tree }}$ over infinite trees $T_{\bar{w}}$. First note that being an outer node is expressible: since $O_{\leftarrow} \top$ is true in right children of matched calls, then

$$
\alpha_{\text {outer }}=\neg\left(\top \mathbf{S}_{\downarrow}\left(\bigcirc_{\leftarrow} \top\right)\right)
$$

is true if no node on the path to the root is inside a call, that is, precisely in outer nodes.

Next note that for each rank- $m$ type $\tau$ of a tree there is a TL ${ }^{\text {tree }}$ formula $\beta_{\tau}$ such that if $s=\iota_{\mathrm{w}-\mathrm{t}}(i)$ is an outer node of $T_{\bar{w}}$, then $\left(T_{\bar{w}}, s\right) \models \beta_{\tau}$ iff the rank- $m$ type of $t^{\bar{w}}(i)$ is $\tau$. If $i$ is not a matched call, then such a type is uniquely determined by $i$ 's label and perhaps pcall or pret, and thus is definable in $\mathrm{TL}^{\text {tree }}$.

If $i$ is a matched call, the existence of such a formula $\beta_{\tau}$ follows from the fact that the rank- $m$ type of $t^{\bar{w}}(i)$ is completely determined by the label of $i$ and the rank- $m$ type $\tau^{\prime}$ of the subtree $t_{0}^{\bar{w}}(i)$ of $t^{\bar{w}}(i)$ rooted at the right child of $s$ (recall that the root only has a right child, by the definition of $\left.t^{\bar{w}}(i)\right)$. Type $\tau^{\prime}$ is expressible in FO and, since $t^{\bar{w}}(i)$ is finite, by Lemma 4.5 it is expressible by a $\mathrm{TL}^{\text {tree }}$ formula $\beta_{\tau^{\prime}}^{\prime \prime}$. If we now inductively take conjunction of every subformula in $\beta_{\tau^{\prime}}^{\prime \prime}$ with $\neg \alpha_{\text {outer }}$, we obtain a formula $\beta_{\tau^{\prime}}^{\prime}$ such that $\left(T_{\bar{w}}, s \cdot 1\right) \models \beta_{\tau^{\prime}}^{\prime}$ 
iff $t_{0}^{\bar{w}}(i) \models \beta_{\tau^{\prime}}^{\prime \prime}$ iff the rank- $m$ type of $t_{0}^{\bar{w}}(i)$ is $\tau^{\prime}$. Hence, $\beta_{\tau}$ is expressible in TL $\mathrm{L}^{\text {tree }}$ as a Boolean combination of propositional letters from $\Sigma$ and formulas $\bigcirc_{\downarrow} \beta_{\tau^{\prime}}^{\prime}$. Note that in this case, $\beta_{\tau}$ does not use pcall and pret.

By Claim 4.8, we need to express, for each node $s=\iota_{\mathrm{w}-\mathrm{t}}(i)$, the rank- $m$ types of $s_{m}^{\overleftarrow{w}}(\bar{w}, s)$ and $s_{m}(\bar{w}, s)$ in $\mathrm{TL}^{\text {tree }}$ over $T_{\bar{w}}$, as well as the rank- $m$ type of $\tau^{\bar{w}}(i)$, in order to express a quantifier-rank $m$ formula, as it will be a Boolean combination of such formulas. Given $s$, we need to define $\iota_{\mathrm{w}-\mathrm{t}}\left(i_{p}\right)$ - the outer position in whose scope $s$ occurs - and then from that point evaluate two FO formulas, defining rank- $m$ types of words over the alphabet of rank- $m$ types of finite trees. By Kamp's theorem [14, each such FO formula is equivalent to an LTL formula whose propositional letters are rank- $m$ types of trees.

Assume we have an LTL formula $\gamma$ expressing the rank- $m$ type $\tau_{0}$ of $\left.s_{m} \vec{w}, s\right)$. By Kamp's theorem and the separation property for LTL, it is written using only propositional letters, Boolean connectives, $\bigcirc$ and $\mathbf{U}$ (that is, no $\odot$ and $\mathbf{S}$ ). We now inductively take conjunction of each subformula of $\gamma$ with $\neg\left(\mathrm{O}_{\leftarrow} T\right)$ (i.e., a $\mathrm{TL}^{\text {tree }}$ formula which is true in left successors), replace LTL connectives $\bigcirc$ and $\mathbf{U}$ by $\bigcirc_{\downarrow}$ and $\mathbf{U}_{\downarrow}$, and replace each propositional letter $\tau$ by $\beta_{\tau}$, to obtain a TL tree formula $\gamma^{\prime}$. Then $\left(T_{\bar{w}}, \iota_{\mathrm{w}-\mathrm{t}}\left(i_{p}\right)\right) \models \gamma^{\prime}$ iff $s_{m}(\bar{w}, s)$ has type $\tau_{0}$. Thus, for a formula

$$
\gamma^{\prime \prime}=\left(\alpha_{\text {outer }} \wedge \gamma^{\prime}\right) \vee \neg \alpha_{\text {outer }} \mathbf{S}_{\downarrow}\left(\alpha_{\text {outer }} \wedge \gamma^{\prime}\right)
$$

is true in $\left(T_{\bar{w}}, \iota_{\mathrm{w}-\mathrm{t}}(i)\right)$ iff the rank- $m$ type of $s_{m}(\bar{w}, s)$ is $\tau_{0}$.

The proof for $s_{m}^{\leftarrow}(\bar{w}, s)$ is similar. Since this word is finite, by Kamp's theorem and the separation property, there is an LTL formula $\gamma$ that uses $\ominus$, S, propositional letters and Boolean connectives such that $\gamma$ evaluated in the last position of the word expresses its rank- $m$ type. Since there is exactly one path from each node to the root, to translate $\gamma$ into a $\mathrm{TL}^{\text {tree }}$ formula $\gamma^{\prime}$ we just need to replace propositional letters by the corresponding formulas $\beta_{\tau}$, and $\ominus$ by $\bigcirc_{\uparrow}$. Then, as for the case of $s_{m}(\bar{w}, s)$, we have that $\gamma^{\prime}$ evaluated in $\iota_{\mathrm{w}-\mathrm{t}}\left(i_{p}\right)$ expresses the type of $s_{m}^{\overleftarrow{w}}(\bar{w}, s)$. Then finally the same formula as in the case of $\left.s_{m} \vec{w}, s\right)$ evaluated in $s$ expresses that type.

Finally we need a TL ${ }^{\text {tree }}$ formula that expresses $\tau_{m}^{\bar{w}}(i)$, the rank- $m$ type of $t^{\bar{w}}(i)$, when evaluated in $\left(T_{\bar{w}}, \iota_{\mathrm{w}-\mathrm{t}}(i)\right)$. We can split this into two cases. If $\alpha_{\text {outer }}$ is true in $\iota_{\mathrm{w}-\mathrm{t}}(i)$, then, as explained earlier, the rank- $m$ type of $t^{\bar{w}}(i)$ is a Boolean combination of propositional letters, and thus definable.

So we now consider the case when $\alpha_{\text {outer }}$ is not true in $\iota_{\mathrm{w}-\mathrm{t}}(i)$. Then $\tau_{m}^{\bar{w}}(i)$ is given by a Boolean combination of formulas that specify (1) the label of $i_{p}$, and (2) the rank- $m$ type of the subtree of $t^{\bar{w}}\left(i_{p}\right)$ rooted at the right child of $\iota_{\mathrm{w}-\mathrm{t}}\left(i_{p}\right)$ with $s$ as a distinguished node. This type can be expressed by a formula $\gamma$ in TL tree over $t_{0}^{\bar{w}}\left(i_{p}\right)$ by [19. Hence if in $\gamma$ we recursively take the conjunction of each subformula with $\neg \alpha_{\text {outer }}$, we obtain a formula $\gamma^{\prime}$ of TL tree that expresses the type of $\left(t_{0}^{\bar{w}}\left(i_{p}\right), s\right)$ when evaluated in $\left(T_{\bar{w}}, s\right)$. Thus, $\tau^{\bar{w}}(i)$ is expressible by a Boolean combination of formulas $\gamma^{\prime}$ and $\neg \alpha_{\text {outer }} \mathbf{S}_{\downarrow}\left(\alpha_{\text {outer }} \wedge a\right)$ where $a$ is a propositional letter.

This completes the proof of translation of FO into TL ${ }^{\text {tree }}$ over nested $\omega$-words, and thus the proof of Lemma 4.4 and Theorem 4.1 .

Recall that $\mathrm{FO}^{k}$ stands for a fragment of $\mathrm{FO}$ that consists of formulas which use at most $k$ variables in total. First, from our translation from NWTL to FO we get:

Corollary 4.9. Over nested words, every FO formula with at most one free variable is equivalent to an $\mathrm{FO}^{3}$ formula. 
It is well known that LTL over $\omega$-words has the separation property, and in particular, every LTL formula is equivalent to an LTL formula without the past connectives when evaluated in the first position of an $\omega$-word. In the case of nested words, however, the situation is quite different from LTL. The following proposition shows that past connectives are necessary even when one evaluates formulae in the first position of a nested word. We let NWTL ${ }^{\text {future }}$ be the future fragment of NWTL (i.e. the fragment that does not use $\mathbf{S}^{\sigma}$ and the operators $\ominus$ and $\Theta_{\mu}$ ).

Proposition 4.10. There are FO sentences over nested words that cannot be expressed in NWTL future.

Proof. We shall look at finite nested words; the proof for the infinite case applies verbatim. To evaluate a formula $\varphi$ of NWTL ${ }^{\text {future }}$ in position $i$ of a nested word $\bar{w}$ of length $n$ one only needs to look at $\bar{w}[i, n]$. That is, if $\bar{w}$ and $\bar{w}^{\prime}$ of length $n$ and $n^{\prime}$ respectively are such that $\bar{w}[i, n] \cong \bar{w}\left[i^{\prime}, n^{\prime}\right]$, then $(\bar{w}, i) \models \varphi$ iff $\left(\bar{w}^{\prime}, i^{\prime}\right) \models \varphi$ for every formula $\varphi$ of NWTL future.

Furthermore, for every collection of NWTL ${ }^{\text {future }}$ formulas $\Psi=\left\{\psi_{1}, \ldots, \psi_{l}\right\}$, one can find a number $k=k(\Psi)$ such that

$$
\bar{w}[i, n] \equiv_{k} \bar{w}\left[i^{\prime}, n^{\prime}\right] \quad \text { implies } \quad(\bar{w}, i) \models \psi_{p} \Leftrightarrow\left(\bar{w}^{\prime}, i^{\prime}\right) \models \psi_{p}, \quad \text { for all } p \leq l .
$$

In particular, if $b^{r}$ stands for the word of length $r$ in which all positions are labeled $b$ and the matching relation is empty, there are numbers $k_{1}>k_{2}$ depending only on $\Psi$, such that

$$
b^{k_{1}} \models \psi_{p} \Leftrightarrow b^{k_{2}} \models \psi_{p}, \quad \text { for all } p \leq l .
$$

Now consider the following NWTL formula:

$$
\alpha=\bigcirc_{\mu} \top \wedge \bigcirc_{\mu} \odot a,
$$

saying that the first position is a call, and the predecessor of its matching return is labeled $a$. We claim that this is not expressible in NWTL ${ }^{\text {future }}$.

Assume to the contrary that there is a formula $\beta$ of NWTL ${ }^{\text {future }}$ equivalent to $\alpha$. Let $\Psi$ be the collection of all subformulas of $\beta$, including $\beta$ itself, and let $k_{1}$ and $k_{2}$ be constructed as above. We now consider two nested words $\bar{w}_{1}$ and $\bar{w}_{2}$ of length $k_{1}+2$ whose underlying words are $b a b^{k_{1}}$ of length $n=k_{1}+2$, such that the matching relation $\mu_{1}$ of $\bar{w}_{1}$ has one edge $\mu_{1}(1,3)$, and the matching relation $\mu_{2}$ of $\bar{w}_{2}$ has one edge $\mu_{2}\left(1, n+1-k_{2}\right)$. In other words, the only return position of $\bar{w}_{1}$ is $r_{1}=3$, and the only return position of $\bar{w}_{2}$ is $r_{2}=n+1-k_{2}$, and thus $\bar{w}_{1}\left[r_{1}, n\right]=b^{k_{1}}$ and $\bar{w}_{2}\left[r_{2}, n\right]=b^{k_{2}}$. Further notice that for every $i>1$ we have $\bar{w}_{1}[i, n] \cong \bar{w}_{2}[i, n]$.

Observe that $\left(\bar{w}_{1}, 1\right) \models \alpha$ and $\left(\bar{w}_{2}, 1\right) \models \neg \alpha$.

We now prove by induction on formulas in $\Psi$ that for each such formula $\gamma$ we have $\left(\bar{w}_{1}, 1\right) \models \gamma$ iff $\left(\bar{w}_{2}, 1\right) \models \gamma$, thus proving that $\beta$ and $\alpha$ cannot be equivalent.

- The base case of propositional letters is immediate.

- The Boolean combinations are straightforward too.

- Let $\gamma=\bigcirc \psi$. Then

$$
\begin{aligned}
& \left(\bar{w}_{1}, 1\right) \models \gamma \\
\Leftrightarrow & \left(\bar{w}_{1}, 2\right) \models \psi \\
\Leftrightarrow \quad & \left(\bar{w}_{2}, 2\right) \models \psi \\
\Leftrightarrow \quad & \left(\bar{w}_{2}, 1\right) \models \gamma,
\end{aligned}
$$

since $\bar{w}_{1}[2, n] \cong \bar{w}_{2}[2, n]$. 
- Let $\gamma=\bigcirc_{\mu} \psi$. Then

$$
\begin{aligned}
& \left(\bar{w}_{1}, 1\right) \models \gamma \\
\Leftrightarrow & \left(\bar{w}_{1}, 3\right) \models \psi \\
\Leftrightarrow & b^{k_{1}} \models \psi \\
\Leftrightarrow & b^{k_{2}} \models \psi \\
\Leftrightarrow & \left(\bar{w}_{2}, n+1-k_{2}\right) \models \psi \\
\Leftrightarrow & \left(\bar{w}_{2}, 1\right) \models \gamma,
\end{aligned}
$$

since $\psi \in \Psi$.

- Let $\gamma=\varphi \mathbf{U}^{\sigma} \psi$. Assume $\left(\bar{w}_{1}, 1\right) \models \gamma$. Consider three cases.

Case 1: $\left(\bar{w}_{1}, 1\right) \models \psi$. By the hypothesis $\left(\bar{w}_{2}, 1\right) \models \psi$ and we are done.

Case 2: The witness for $\varphi \mathbf{U}^{\sigma} \psi$ occurs beyond the only return. Then $\left(\bar{w}_{1}, 1\right) \models \varphi$ and $\left(\bar{w}_{1}, r_{1}\right) \models \varphi \mathbf{U}^{\sigma} \psi$. Since $\varphi \mathbf{U}^{\sigma} \psi \in \Psi$ we have $\left(\bar{w}_{2}, r_{2}\right) \models \varphi \mathbf{U}^{\sigma} \psi$, and by the hypothesis, $\left(\bar{w}_{2}, 1\right) \models \varphi$, so $\left(\bar{w}_{2}, 1\right) \models \varphi \mathbf{U}^{\sigma} \psi$.

Case 3: The witness for $\varphi \mathbf{U}^{\sigma} \psi$ occurs inside the call. Since for every position $i>1$ we have $\left(\bar{w}_{1}, i\right) \models \varphi$ iff $\left(\bar{w}_{2}, i\right) \models \varphi$ and likewise for $\psi$, the same summary path witnesses $\varphi \mathbf{U}^{\sigma} \psi$ in $\bar{w}_{2}$.

Thus, $\left(\bar{w}_{2}, 1\right) \models \gamma$.

Now assume $\left(\bar{w}_{2}, 1\right) \models \gamma$. In the proof of $\left(\bar{w}_{1}, 1\right) \models \gamma$ is the same as above in Cases 1 and 2. For Case 3, assume that in the path which is a witness for $\varphi \mathbf{U}^{\sigma} \psi$ the position in which $\psi$ is true is the 2 nd or the 3 rd position in the word. Then the same path witnesses $\left(\bar{w}_{1}, 1\right) \models \gamma$, as in the proof of Case 3 above. Next assume it is a position with index $j$ higher than 3 (which is still labeled $b$ ) where $\psi$ first occurs. Then $\varphi$ must be true in all positions $i$ with $3 \leq i \leq j$ in $\bar{w}_{2}$. Hence $\varphi$ is true in all such positions in $\bar{w}_{1}$ as well, and thus the summary path in $\bar{w}_{1}$ that skips the first call (i.e. jumps from 1 to 3 ) witnesses $\varphi \mathbf{U}^{\sigma} \psi$. Hence, in all the cases $\left(\bar{w}_{2}, 1\right) \models \gamma$ implies $\left(\bar{w}_{1}, 1\right) \models \gamma$, which completes the inductive proof, and thus shows the inexpressibility of $\alpha$ in NWTL future.

Note also that adding all other until/since pairs to NWTL does not change its expressiveness. That is, if we let $\mathrm{NWTL}^{+}$be NWTL $+\left\{\mathbf{U}, \mathbf{S}, \mathbf{U}^{c}, \mathbf{S}^{c}, \mathbf{U}^{a}, \mathbf{S}^{a}\right\}$, then:

Corollary 4.11. $\mathrm{NWTL}^{+}=\mathrm{FO}$.

Later, when we provide our upper bounds for model-checking, we shall pride the upper bounds with respect to $\mathrm{NWTL}^{+}$rather than just NWTL.

Remark In the conference version, we had a corollary stating that the since operator can be eliminated for formulae evaluated in the first position of a nested word. It relied on the proof of Theorem 4.1 and the separation property for $\mathrm{TL}^{\text {tree }}$ claimed in [19]. The latter, as was discovered recently, is incorrect. The proof of Theorem 4.1 relies only on the expressive completeness of $\mathrm{TL}^{\text {tree }}$ which is correct [26, 20] and thus is not affected.

4.2. The within operator. We now go back to the three until/since operators originally proposed for temporal logics on nested words, based on the the linear, call, and abstract paths. In other words, our basic logic, denoted by $\mathrm{LTL}^{\mu}$, is

$$
\begin{aligned}
& \varphi, \varphi^{\prime}:=\top|a| \text { call } \mid \text { ret }|\neg \varphi| \varphi \vee \varphi^{\prime} \mid \\
& \bigcirc \varphi\left|\bigcirc_{\mu} \varphi\right| \Theta\left|\Theta_{\mu} \varphi\right| \\
& \varphi \mathbf{U} \varphi^{\prime}\left|\varphi \mathbf{S} \varphi^{\prime}\right| \varphi \mathbf{U}^{c} \varphi^{\prime}\left|\varphi \mathbf{S}^{c} \varphi^{\prime}\right| \varphi \mathbf{U}^{a} \varphi^{\prime} \mid \varphi \mathbf{S}^{a} \varphi^{\prime}
\end{aligned}
$$

We now extend this logic with the within $\mathcal{W}$ operator proposed in [2. Recall that $(\bar{w}, i) \models \mathcal{W} \varphi$ iff $i$ is a call, and $(\bar{w}[i, j], i) \models \varphi$, where $j=r(i)$ if $i$ is a matched call, $j=|\bar{w}|$ 
if $i$ is an unmatched call and $\bar{w}$ is finite, and $j=\infty$ otherwise. We denote this extended logic by $\mathrm{LTL}^{\mu}+\mathcal{W}$.

Theorem 4.12. $\mathrm{LTL}^{\mu}+\mathcal{W}=\mathrm{FO}$ over both finite and infinite nested words.

Proof. The translation from $\mathrm{LTL}^{\mu}+\mathcal{W}$ into $\mathrm{FO}$ is similar to the translation used in the proof of Theorem 4.13. To prove the other direction, we show how to translate NWTL ${ }^{s}$ into $\mathrm{LTL}^{\mu}+\mathcal{W}$. Recall that by Lemma 4.4, we know that $\mathrm{NWTL}^{s}=\mathrm{FO}$ over both finite and infinite nested words. More precisely, for every formula $\varphi$ in $\mathrm{NWTL}^{s}$, we show how to construct a formula $\alpha_{\varphi}$ in $\mathrm{LTL}^{\mu}+\mathcal{W}$ such that for every nested word $\bar{w}$ (finite or infinite) and position $i$ in it, we have that $(\bar{w}, i) \models \varphi$ if and only if $(\bar{w}, i) \models \alpha_{\varphi}$.

Since $\mathrm{LTL}^{\mu}$ includes the same past modalities as $\mathrm{NWTL}^{s}, \alpha_{\varphi}$ is trivial to define for the atomic formulas, Boolean combinations and next and previous modalities:

$$
\begin{aligned}
& \alpha_{\top}:=\top, \\
& \alpha_{\text {call }}:=\text { call, } \\
& \alpha_{\text {ret }}:=\text { ret, } \\
& \alpha_{a}:=a, \\
& \alpha_{\neg \varphi}:=\neg \alpha_{\varphi}, \\
& \alpha_{\varphi \vee \psi}:=\alpha_{\varphi} \vee \alpha_{\psi} \text {, } \\
& \alpha_{\bigcirc \varphi}:=\bigcirc \alpha_{\varphi}, \\
& \alpha_{\bigcirc_{\mu} \varphi}:=\bigcirc_{\mu} \alpha_{\varphi}, \\
& \alpha_{\Theta \varphi}:=\ominus \alpha_{\varphi}, \\
& \alpha_{\Theta_{\mu} \varphi}:=\Theta_{\mu} \alpha_{\varphi} .
\end{aligned}
$$

Thus, we only need to show how to define $\alpha_{\varphi} \mathbf{U}_{s}^{\sigma} \psi$ and $\alpha_{\varphi} \mathbf{S}_{s}^{\sigma} \psi$. Formula $\alpha_{\varphi} \mathbf{U}_{s}^{\sigma} \psi$ is defined as:

$$
\begin{aligned}
\alpha_{\varphi} \mathbf{U}_{s}^{\sigma} \psi & :=\left[\operatorname{mret} \wedge \alpha_{\psi}\right] \vee\left[\neg \operatorname { m r e t } \wedge \left(\left(\beta_{\varphi} \mathbf{U}^{a}\left(\neg \text { mret } \wedge \alpha_{\psi}\right)\right) \vee\right.\right. \\
& \left.\left.\left(\beta_{\varphi} \mathbf{U}^{a}\left(\beta_{\varphi} \wedge \bigcirc\left(\operatorname{mret} \wedge \alpha_{\psi}\right)\right)\right) \vee\left(\beta_{\varphi} \mathbf{U}^{a} \mathcal{W} \diamond\left(\alpha_{\psi} \wedge \ominus\left(\gamma \mathbf{S}^{c}\left(\alpha_{\varphi} \wedge \neg \Theta \top\right)\right)\right)\right)\right)\right]
\end{aligned}
$$

where mret is defined as ret $\wedge \Theta_{\mu} \top$, to capture matching return positions, $\diamond \theta$ is defined as $\top \mathbf{U} \theta$ and formulas $\beta_{\varphi}, \gamma$ are defined as:

$$
\begin{aligned}
\beta_{\varphi} & :=\alpha_{\varphi} \vee \text { mret }, \\
\gamma & :=\beta_{\varphi} \mathbf{S}^{a}\left(\alpha_{\varphi} \wedge \neg \text { ret } \wedge \ominus\left(\left(\alpha_{\varphi} \wedge \neg \text { ret }\right) \text { Scall }\right)\right) .
\end{aligned}
$$

Moreover, formula $\alpha_{\varphi \mathbf{S}_{s}^{\sigma} \psi}$ is defined as:

$$
\alpha_{\varphi} \mathbf{S}_{s}^{\sigma} \psi:=\alpha_{\psi} \vee\left(\alpha_{\varphi} \wedge \ominus\left(\gamma \mathbf{S}^{c}\left(\beta_{\varphi} \mathbf{S}^{a}\left(\alpha_{\psi} \wedge \neg \mathrm{mret}\right)\right)\right)\right)
$$

This concludes the proof of the theorem. 
4.3. CaRet and other within operators. The logic CaRet, as defined in [2], did not have all the operators of $\mathrm{LTL}^{\mu}$. In fact it did not have the previous operators $\ominus$ and $\Theta_{\mu}$, and it only had linear and abstract until operators, and the call since operator. That is, CaRet was defined as

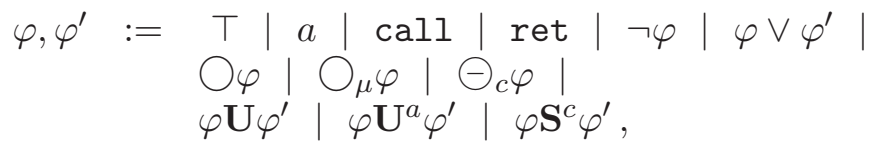

and we assume that $a$ ranges over $\Sigma \cup\{$ pret $\}$, where pret is true in pending returns. Notice that pret is not expressible with the remaining operators. Recall that the operator $\Theta_{c}$ is the previous operator corresponding to call paths; formally, $(\bar{w}, i) \models \Theta_{c} \varphi$ iff $\mathcal{C}(i)$ is defined and $(\bar{w}, \mathcal{C}(i)) \models \varphi$.

A natural question is whether there is an expressively-complete extension of this logic. It turns out that the past modality $\ominus$, together with two within operators based on $\mathcal{C}$ and $\mathcal{R}$ (the innermost call and its return) functions provide such an extension. We define two new formulas $\mathcal{C} \varphi$ and $\mathcal{R} \varphi$ with the semantics as follows:

- $(\bar{w}, i) \models \mathcal{C} \varphi$ iff $(\bar{w}[j, i], j) \models \varphi$, where $j=\mathcal{C}(i)$ if $\mathcal{C}(i)$ is defined, and $j=1$ otherwise.

- $(\bar{w}, i) \models \mathcal{R} \varphi$ if $(\bar{w}[i, j], i) \models \varphi$, where $j=\mathcal{R}(i)$ if $\mathcal{R}(i)$ is defined, and $j=|\bar{w}|$ (if $\bar{w}$ is finite) or $\infty$ (if $\bar{w}$ is infinite) otherwise.

The logic obtained by adding $\mathcal{C}$ and $\mathcal{R}$ to CaRet is denoted by CaRet $+\{\mathcal{C}, \mathcal{R}\}$.

Theorem 4.13. CaRet $+\{\mathcal{C}, \mathcal{R}\}=$ FO over both finite and infinite nested words.

As a corollary (to the proof) we obtain the following:

Corollary 4.14. For every FO formula $\varphi(x)$ over finite or infinite nested words, there is a formula $\psi$ of CaRet $+\{\mathcal{C}, \mathcal{R}\}$ that does not use the $\mathbf{U}^{a}$ operator, such that $\bar{w} \models \varphi(i)$ iff $(\bar{w}, i) \models \psi$.

The proof of this result is somewhat involved, and relies on different techniques. The operators used in CaRet do not correspond naturally to tree translations of nested words, and the lack of all until/since pairs makes a translation from NWTL hard. We thus use a composition argument directly on nested words. The theorem is proved for finite nested words, but the same techniques can be used to prove the infinite case.

We extend the vocabulary with two constants min and max, and assume that min is always interpreted as the first element of the nested word and max as the last element.

Let $\bar{w}$ be a finite nested word of length $n$ and and $i$ an element in $\bar{w}$. Let $c_{1}, \ldots, c_{m}$, where $m \geq 0$, be all elements in $\bar{w}$ such that, for each $j \in[1, m], c_{j}<i$ and there is an element $r_{j}$ such that $\mu\left(c_{j}, r_{j}\right)$ and $i \leq r_{j}$. Assume without loss of generality that $c_{1}<c_{2}<\cdots<c_{m}$.

Fix $k \geq 0$. Let $\Gamma$ be the set of all rank- $k$ types of nested words with distinguished constants min and max (including the rank- $k$ type of the empty nested word). We define the word $\Omega_{k}(\bar{w}, i)=a_{0} a_{1} \cdots a_{m}$ over alphabet $\Gamma \times \Gamma$ as follows:

- The element $a_{0}$ is labeled with the tuple whose first component is the rank- $k$ type of $\left(\bar{w}\left[1, c_{1}-1\right], \min , \max \right)$ and whose second component is the rank- $k$ type of $\left(\bar{w}\left[r_{1}+\right.\right.$ $1, n]$, min, $\max$ ) if $m \neq 0$ (notice that if $c_{1}=1$ then $\bar{w}\left[1, c_{1}-1\right]$ is the empty nested word, and the same is true of $\bar{w}\left[r_{i+1}, n\right]$ if $\left.r_{m}=n\right)$; otherwise, it is labeled with the tuple whose first component is the rank- $k$ type of $(\bar{w}[1, i-1], \min , \max )$ and whose second component is the rank- $k$ type of $(\bar{w}[i, n], \min , \max )$ (notice that if $i=1$ then $\bar{w}[1, i-1]$ is the empty nested word); 
- for each $0<j<m$, the element $a_{j}$ is labeled with the tuple whose first component is the rank- $k$ type of $\left(\bar{w}\left[c_{j}, c_{j+1}-1\right], \min , \max \right)$ and whose second component is the rank- $k$ type of $\left(\bar{w}\left[r_{j+1}+1, r_{j}\right], \min , \max \right)$; and

- if $m \neq 0$ then the element $a_{m}$ is labeled with the the tuple whose first component is the rank- $k$ type of $\left(\bar{w}\left[c_{m}, i-1\right], \min , \max \right)$ and whose second component is the rank- $k$ type of $\left(\bar{w}\left[i, r_{m}\right]\right.$, min, $\left.\max \right)$.

The following is our composition argument:

Lemma 4.15 (Composition Method). Let $\bar{w}_{1}$ and $\bar{w}_{2}$ be two nested words, and let $i$ and $i^{\prime}$ be two elements in $\bar{w}_{1}$ and $\bar{w}_{2}$, respectively. Then $\Omega_{k}\left(\bar{w}_{1}, i\right) \equiv_{k+2} \Omega_{k}\left(\bar{w}_{2}, i^{\prime}\right)$ implies $\left(\bar{w}_{1}, i, \min , \max \right) \equiv_{k}\left(\bar{w}_{2}, i^{\prime}, \min , \max \right)$.

Proof. First we need to introduce some terminology. Let $\bar{w}$ be a finite nested word of length $n$ and $i$ be a position in $\bar{w}$. Assume elements $c_{1}, \ldots, c_{m}, r_{1}, \ldots, r_{m}$ are defined as above. With each element $s$ of $\bar{w}$ we associate an element $[s]$ of $\Omega_{k}(\bar{w}, i)$ as follows:

- If $m \neq 0$ and $s$ belongs to $\bar{w}\left[1, c_{1}-1\right]$ or $\bar{w}\left[r_{1}+1, n\right]$, then $[s]$ is the first element of $\Omega_{k}(\bar{w}, i)$. In such case we say that $\bar{w}\left[0, c_{1}-1\right]$ and $\bar{w}\left[r_{1}+1, n\right]$ are the left and right intervals represented by $[s]$, respectively.

If $m=0$ and $s$ is an arbitrary element of $\bar{w}$, then $[s]$ is also the first (and unique) element of $\Omega_{k}(\bar{w}, i)$. In such case we say that $\bar{w}[0, i-1]$ and $\bar{w}[i, n]$ are the left and right intervals represented by $[s]$, respectively.

- If $m \neq 0$ and $s$ belongs to $\bar{w}\left[c_{m}, i-1\right]$ or $\bar{w}\left[i, r_{m}\right]$, then $[s]$ is the last element of $\Omega_{k}(\bar{w}, i)$. In such case we say that $\bar{w}\left[c_{m}, i-1\right]$ and $\bar{w}\left[i, r_{m}\right]$ are the left and right intervals represented by $[s]$, respectively.

- If $m \neq 0$ and $s$ belongs to $\bar{w}\left[c_{\ell}, c_{\ell+1}-1\right]$ or $\bar{w}\left[r_{\ell+1}+1, r_{\ell}\right]$, for some $1 \leq \ell<m$, then $[s]$ is the $(\ell+1)$-th element of $\Omega_{k}(\bar{w}, i)$. In such case we say that $\bar{w}\left[c_{\ell}, c_{\ell+1}-1\right]$ and $\bar{w}\left[r_{\ell+1}+1, r_{\ell}\right]$ are the left and right intervals represented by $[s]$, respectively.

We denote by $[s]^{L}$ and $[s]^{R}$ the left and right intervals represented by $[s]$, respectively.

We now prove the lemma. For each round $j(0 \leq j \leq k)$ of the $k$-round game on $\left(\bar{w}_{1}, i, \min , \max \right)$ and $\left(\bar{w}_{2}, i^{\prime}, \min , \max \right)$, Player II's response $b_{j}$ in $\bar{w}_{2}$ to an element $a_{j}$ in $\bar{w}_{1}$, played by Player $\mathrm{I}$ is defined as follows (the strategy for the case when Player I picks a point in $\bar{w}_{2}$ is completely symmetric). Assume that Player I plays element $\left[a_{j}\right]$ in $\Omega_{k}\left(\bar{w}_{1}, i\right)$ in round $j$ of the $(k+2)$-round game on $\Omega_{k}\left(\bar{w}_{1}, i\right)$ and $\Omega_{k}\left(\bar{w}_{2}, i^{\prime}\right)$. Then given that $\Omega_{k}\left(\bar{w}_{1}, i\right) \equiv_{k+2} \Omega_{k}\left(\bar{w}_{2}, i^{\prime}\right)$, Player II uses her winning strategy to choose a response $\left[q_{j}\right]$ in $\Omega_{k}\left(\bar{w}_{2}, i^{\prime}\right)$ to $\left[a_{j}\right]$. Thus, by definition of $\Omega_{k}$, we have that the right and left intervals represented by $\left[a_{j}\right]$ have the same rank- $k$ type as the right and left intervals represented by $\left[q_{j}\right]$, respectively. Hence, if $a_{j}$ belongs to the left interval represented by $\left[a_{j}\right]$, then the Player II can find response $b_{j}$ to $a_{j}$ according to the winning strategy for the $k$-round game on $\left[a_{j}\right]^{L}$ and $\left[q_{j}\right]^{L}$, and if $a_{j}$ belongs to the right interval represented by $\left[a_{j}\right]$, then the Player II can find response $b_{j}$ to $a_{j}$ according to the winning strategy for the $k$-round game on $\left[a_{j}\right]^{R}$ and $\left[q_{j}\right]^{R}$.

Assume that for round $0 \leq j<k$ the elements played by following this strategy are (1) $\left(\left[p_{1}\right], \ldots,\left[p_{j}\right]\right)$ in $\Omega_{k}\left(\bar{w}_{1}, i\right),(2)\left(\left[q_{1}\right], \ldots,\left[q_{j}\right]\right)$ in $\Omega_{k}\left(\bar{w}_{2}, i^{\prime}\right),(3)\left(a_{1}, \ldots, a_{j}\right)$ in $\bar{w}_{1}$, and $(4)$ $\left(b_{1}, \ldots, b_{j}\right)$ in $\bar{w}_{2}$. We note that by definition of the strategy, for every $i \in[1, j]$, we have that $a_{i}=p_{i}$ or $b_{i}=q_{i}$. Since we assume that the $\left[p_{j}\right]$ 's and $\left[q_{j}\right]$ 's are played according to a winning strategy for Player II in the $(k+2)$-round game on $\Omega_{k}\left(\bar{w}_{1}, i\right)$ and $\Omega_{k}\left(\bar{w}_{2}, i^{\prime}\right)$, it is 
the case that:

$$
\begin{aligned}
& \left(\Omega_{k}\left(\bar{w}_{1}, i\right),\left[p_{1}\right], \ldots,\left[p_{j}\right]\right) \quad \equiv_{k-j+2} \\
& \left(\Omega_{k}\left(\bar{w}_{2}, i^{\prime}\right),\left[q_{1}\right], \ldots,\left[q_{j}\right]\right) .
\end{aligned}
$$

By the way the strategy is defined, for each $\ell \in[1, j]$, if $\bar{a}_{\ell}^{L}$ and $\bar{a}_{\ell}^{R}$ are the subtuples of $\left(a_{1}, \ldots, a_{j}\right)$ containing the elements from $\left(a_{1}, \ldots, a_{j}\right)$ that belong to $\left[a_{\ell}\right]^{L}$ and $\left[a_{\ell}\right]^{R}$, respectively, then the corresponding subtuples $\bar{b}_{\ell}^{L}$ and $\bar{b}_{\ell}^{R}$ of $\left(b_{1}, \ldots, b_{j}\right)$ contain the elements from $\left(b_{1}, \ldots, b_{j}\right)$ that belong to $\left[b_{\ell}\right]^{L}$ and $\left[b_{\ell}\right]^{R}$, respectively. Further, by definition of the strategy, we also have that $\left(\left[a_{\ell}\right]^{L}, \bar{a}_{\ell}^{L}, \min , \max \right) \equiv_{k-j}\left(\left[b_{\ell}\right]^{L}, \bar{b}_{\ell}^{L}, \min , \max \right)$ and $\left(\left[a_{\ell}\right]^{R}, \bar{a}_{\ell}^{R}, \min , \max \right) \equiv_{k-j}\left(\left[b_{\ell}\right]^{R}, \bar{b}_{\ell}^{R}, \min , \max \right)$.

We now show how to define Player II's response in the round $j+1$. Let us assume without loss of generality that for round $j+1$ of the game on $\left(\bar{w}_{1}, i, \min , \max \right)$ and $\left(\bar{w}_{2}, i^{\prime}, \min , \max \right)$, Player I picks an element $a_{j+1}$ in $\bar{w}_{1}$ that belongs to the left interval represented by $\left[a_{j+1}\right]$ (all the other cases can be treated in a similar way). Player II response $b_{j+1}$ in $\bar{w}_{2}$ is defined as follows. First, there must be an element $[s]$ in $\Omega_{k}\left(\bar{w}_{2}, i^{\prime}\right)$ such that

$$
\begin{aligned}
& \left(\Omega_{k}\left(\bar{w}_{1}, i\right),\left[p_{1}\right], \ldots,\left[p_{j}\right],\left[p_{j+1}\right]\right) \quad \equiv_{k-j+1} \\
& \quad\left(\Omega_{k}\left(\bar{w}_{2}, i^{\prime}\right),\left[q_{1}\right], \ldots,\left[q_{j}\right],[s]\right),
\end{aligned}
$$

where $p_{j+1}=a_{j+1}$. The latter, together with the way that the strategy is defined, implies that there is an element $b$ in $[s]^{L}$ such that $\left(\left[a_{j+1}\right]^{L}, \bar{a}^{\prime}, a_{j+1}, \min , \max \right) \equiv_{k-j-1}$ $\left([s]^{L}, \bar{b}^{\prime}, b, \min , \max \right)$, where $\bar{a}^{\prime}$ is the subtuple of $\left(a_{1}, \ldots, a_{j}\right)$ containing all the elements in $\left(a_{1}, \ldots, a_{j}\right)$ that belong to $\left[a_{j+1}\right]^{L}$ and $\bar{b}^{\prime}$ is the corresponding subtuple of $\left(b_{1}, \ldots, b_{j}\right)$. We then set $b_{j+1}=b$.

We show by induction that, for each $j \leq k$, if $\left(a_{1}, \ldots, a_{j}\right)$ and $\left(b_{1}, \ldots, b_{j}\right)$ are the first $j$ moves played by Player I and Player II on $\left(\bar{w}_{1}, i, \min , \max \right)$ and $\left(\bar{w}_{2}, i^{\prime}, \min , \max \right)$, respectively, according to the strategy defined above, then $\left(\left(a_{1}, \ldots, a_{j}\right),\left(b_{1}, \ldots, b_{j}\right)\right)$ defines a partial isomorphism between $\left(\bar{w}_{1}, i, \min , \max \right)$ and $\left(\bar{w}_{2}, i^{\prime}, \min , \max \right)$. This is sufficient to show that $\left(\bar{w}_{1}, i, \min , \max \right) \equiv_{k}\left(\bar{w}_{2}, i^{\prime}, \min , \max \right)$.

Assume $j=0$. Since $\Omega_{k}\left(\bar{w}_{1}, i\right) \equiv_{k+2} \Omega_{k}\left(\bar{w}_{2}, i^{\prime}\right)$, it must be th case that the labels of the last elements of $\Omega_{k}\left(\bar{w}_{1}, i\right)$ and $\Omega_{k}\left(\bar{w}_{2}, i^{\prime}\right)$ coincide. Thus, $\left([i]^{R}, i\right) \equiv_{0}\left(\left[i^{\prime}\right]^{R}, i^{\prime}\right)$, and we conclude that $i$ and $i^{\prime}$ have the same label, and $i$ is a call (resp. return) iff $i^{\prime}$ is a call (resp. return). Further, if $i=$ min then $\Omega_{k}\left(\bar{w}_{1}, i\right)$ has only one element and that element is labeled $\left(\tau_{\varepsilon}, \tau\right)$, for some $\tau \neq \tau_{\varepsilon}$. Since $\Omega_{k}\left(\bar{w}_{1}, i\right) \equiv_{k+2} \Omega_{k}\left(\bar{w}_{2}, i^{\prime}\right), \Omega_{k}\left(\bar{w}_{2}, i\right)$ also has a single element and that element is labeled $\left(\tau_{\varepsilon}, \tau\right)$. It follows that $i^{\prime}=$ min. The converse can be proved analogously. In the same way it is possible to show that $i=\max$ iff $i^{\prime}=\max$.

Assume that the property holds for $j$. Also, assume without loss of generality that for the round $j+1$ of the game on $\left(\bar{w}_{1}, i, \min , \max \right)$ and $\left(\bar{w}_{2}, i^{\prime}, \min , \max \right)$, Player I picks an element $a_{j+1}$ in $\bar{w}_{1}$ that belongs to the right interval represented by $\left[a_{j+1}\right]$ (all the other cases can be treated in a similar way). We prove that $b_{j+1}$ as defined above preserves the partial isomorphism. First we show that $a_{j+1}=i$ iff $b_{j+1}=i^{\prime}$. In this case $\left[a_{j+1}\right]$ is the last element of $\Omega_{k}\left(\bar{w}_{1}, i\right)$, and $\Omega_{k}\left(\bar{w}_{1}, i\right) \equiv_{k+2} \Omega_{k}\left(\bar{w}_{2}, i^{\prime}\right)$ implies that $\left[b_{j+1}\right]$ is the last element of $\Omega_{k}\left(\bar{w}_{2}, i^{\prime}\right)$. Since $a_{j+1}=i$ is the first element of $\left[a_{j+1}\right]^{R}, b_{j+1}$ has to be the first element of $\left[b_{j+1}\right]^{R}$, which is $i^{\prime}$.

In the same way it is possible to prove that $a_{j+1}=\min$ iff $b_{j+1}=\min$, and that $a_{j+1}=\max$ iff $b_{j+1}=\max$. 
Further, it is also clear that the label of $a_{j+1}$ in $\bar{w}_{1}$ is $a$ iff the label of $b_{j+1}$ in $\bar{w}_{2}$ is $a$, for each $a \in \Sigma$. Next we consider the remaining cases.

- $a_{j+1} \in \operatorname{call}$. Then $\left(\left[a_{j+1}\right]^{R}, \bar{a}^{\prime}, a_{j+1}, \min , \max \right) \equiv_{k-j-1}\left(\left[b_{j+1}\right]^{R}, \bar{b}^{\prime}, b_{j+1}\right.$, min, max $)$, where $\bar{a}^{\prime}$ is the subtuple of $\left(a_{1}, \ldots, a_{j}\right)$ containing all the elements in $\left(a_{1}, \ldots, a_{j}\right)$ that belong to $\left[a_{j+1}\right]^{L}$ and $\bar{b}^{\prime}$ is the corresponding subtuple of $\left(b_{1}, \ldots, b_{j}\right)$. This immediately implies that $b_{j+1} \in$ call. The converse is proved analogously.

- $a_{j+1} \in$ ret. This is similar to the previous case.

- Suppose first that $a_{j+1}<a_{\ell}$ holds for some $\ell \in[1, j]$. Since $a_{j+1}$ belongs to $\left[a_{j+1}\right]^{R}$, we have that $a_{\ell}$ belongs to $\left[a_{\ell}\right]^{R}$ and, thus, we only need to consider the cases $\left[a_{\ell}\right]=\left[a_{j+1}\right]$ and $\left[a_{j+1}\right]<\left[a_{\ell}\right]$. If $\left[a_{\ell}\right]=\left[a_{j+1}\right]$, then $\left(\left[a_{\ell}\right]^{R}, a_{\ell}, a_{j+1}\right) \equiv_{0}\left(\left[b_{\ell}\right]^{R}, b_{\ell}, b_{j+1}\right)$ and, therefore, $b_{j+1}<b_{l}$ also holds. If $\left[a_{j+1}\right]<\left[a_{\ell}\right]$, then $\left[b_{j+1}\right]<\left[b_{\ell}\right]$ and, thus, $b_{j+1}<b_{\ell}$ holds since $b_{\ell}$ and $b_{j+1}$ belong to $\left[b_{\ell}\right]^{R}$ and $\left[b_{j+1}\right]^{R}$, respectively.

Suppose, on the other hand, that $a_{\ell}<a_{j+1}$ holds for some $\ell \in[1, j]$. We need to consider three cases: $\left[a_{\ell}\right]=\left[a_{j+1}\right],\left[a_{\ell}\right]<\left[a_{j+1}\right]$ and $\left[a_{j+1}\right]<\left[a_{\ell}\right]$. If $\left[a_{\ell}\right]=\left[a_{j+1}\right]$, then $\left(\left[a_{\ell}\right]^{R}, a_{\ell}, a_{j+1}\right) \equiv_{0}\left(\left[b_{\ell}\right]^{R}, b_{\ell}, b_{j+1}\right)$ and, therefore, $b_{\ell}<b_{j+1}$ also holds. If $\left[a_{j+1}\right]>\left[a_{\ell}\right]$, then $a_{\ell}$ belongs to $\left[a_{\ell}\right]^{L}$ and $\left[b_{j+1}\right]<\left[b_{\ell}\right]$ and, thus, $b_{\ell}<b_{j+1}$ holds since $b_{\ell}$ belongs to $\left[b_{\ell}\right]^{L}$ while $b_{j+1}$ belongs to $\left[b_{j+1}\right]^{R}$. Finally, if $\left[a_{\ell}\right]>\left[a_{j+1}\right]$, then $\left[b_{\ell}\right]>\left[b_{j+1}\right]$ and, thus, $b_{\ell}<b_{j+1}$ holds since $b_{j+1}$ belongs to $\left[b_{j+1}\right]^{R}$ and every element in $\left[b_{j+1}\right]^{R}$ is bigger than every element in either $\left[b_{\ell}\right]^{R}$ or $\left[b_{\ell}\right]^{L}$.

The converse is proved analogously.

- Suppose first that $\mu\left(a_{j+1}, a_{\ell}\right)$ holds for some $\ell \in[1, j]$. Since $a_{j+1}$ belongs to the right interval represented by $\left[a_{j+1}\right]$, it is the case that $\left[a_{\ell}\right]$ also belongs to $\left[a_{j+1}\right]^{R}$. Thus, given that $\left(\left[a_{\ell}\right]^{R}, a_{\ell}, a_{j+1}\right) \equiv_{0}\left(\left[b_{\ell}\right]^{R}, b_{\ell}, b_{j+1}\right)$, we conclude that $\mu\left(b_{j+1}, b_{\ell}\right)$ holds.

Second, $\mu\left(a_{\ell}, a_{j+1}\right)$ holds for some $\ell \in[1, j]$. It is not hard to see that $\left[a_{\ell}\right]=$ $\left[a_{j+1}\right]$. We need to consider two cases: If $a_{\ell}$ belongs to $\left[a_{j+1}\right]^{R}$, then $\left(\left[a_{\ell}\right]^{R}, a_{\ell}, a_{j+1}\right) \equiv_{0}$ $\left(\left[b_{\ell}\right]^{R}, b_{\ell}, b_{j+1}\right)$, and thus, $\mu\left(b_{\ell}, b_{j+1}\right)$ holds. If $a_{\ell}$ belongs to $\left[a_{j+1}\right]^{L}$, then $a_{\ell}$ is the first element of $\left[a_{j+1}\right]^{L}$ and $a_{j+1}$ is the last element of $\left[a_{j+1}\right]^{R}$. Thus, since $\left(\left[a_{j+1}\right]^{L}, a_{\ell}, \min \right) \equiv_{0}$ $\left(\left[b_{j+1}\right]^{L}, b_{\ell}, \min \right)$, we conclude that $b_{\ell}$ is the first element of $\left[b_{j+1}\right]^{L}$. Further, since $\left(\left[a_{j+1}\right]^{R}, a_{j+1}, \max \right) \equiv_{0}\left(\left[b_{j+1}\right]^{L}, b_{j+1}, \max \right)$, we conclude that $b_{j+1}$ is the last element of $\left[b_{j+1}\right]^{R}$. Therefore, $\mu\left(b_{\ell}, b_{j+1}\right)$ holds.

The converse is proved analogously.

This concludes the proof of the lemma.

We now present the proof of Theorem 4.13 .

Proof of Theorem 4.13. We first show that every CaRet $+\{\mathcal{R}, \mathcal{C}\}$ formula $\varphi$ is equivalent to an FO formula $\alpha_{\varphi}(x)$ over nested words, that is, for every nested word $\bar{w}$ it is the case that $(\bar{w}, i) \models \varphi$ iff $\bar{w} \models \alpha_{\varphi}(i)$. The translation is standard, and can be done by recursively defining $\alpha_{\varphi}(x)$ from $\varphi$ as shown below. We use the notation $\theta(x)^{[y, z]}$ for the relativization of $\theta(x)$ to elements in the interval $[y, z]$, that is, $\theta(x)^{[y, z]}$ is obtained from $\theta(x)$ by replacing each subformula of the form $\exists u \beta$ with $\exists u(y \leq u \wedge u \leq z \wedge \beta)$ and each subformula of the form $\forall u \beta$ with $\forall u(y \leq u \wedge u \leq z \rightarrow \beta)$. Here is the translation: 


$$
\begin{aligned}
& \alpha_{a}(x):=P_{a}(x), \\
& \alpha_{\text {call }}(x):=\operatorname{call}(x) \text {, } \\
& \alpha_{\text {ret }}(x):=\operatorname{ret}(x) \text {, } \\
& \alpha_{\text {int }}(x):=\neg \operatorname{call}(x) \wedge \neg \operatorname{ret}(x), \\
& \alpha_{\text {pret }}(x):=\operatorname{ret}(x) \wedge \neg \exists y \mu(y, x) \text {, } \\
& \alpha_{\neg \varphi}(x):=\neg \alpha_{\varphi}(x), \\
& \alpha_{\varphi \vee \psi}(x):=\alpha_{\varphi}(x) \vee \alpha_{\psi}(x) \\
& \alpha_{\bigcirc_{\varphi}}(x):=\exists y\left(x<y \wedge \neg \exists z(x<z \wedge z<y) \wedge \alpha_{\varphi}(y)\right), \\
& \alpha_{\Theta_{\varphi}}(x):=\exists y\left(y<x \wedge \neg \exists z(y<z \wedge z<x) \wedge \alpha_{\varphi}(y)\right), \\
& \alpha_{\bigcirc_{\mu} \varphi}(x):=\exists y\left(\mu(x, y) \wedge \alpha_{\varphi}(y)\right) \text {, } \\
& \alpha_{\Theta_{c} \varphi}(x):=\exists y \exists z\left(y<x \wedge x<z \wedge \mu(y, z) \wedge \alpha_{\varphi}(y) \wedge\right. \\
& \forall u \forall v(u<x \wedge x<v \wedge \mu(u, v) \rightarrow u=y \vee u<y)), \\
& \alpha_{\varphi \mathbf{U} \psi}(x):=\exists y\left((x<y \vee x=y) \wedge \alpha_{\psi}(y) \wedge\right. \\
& \left.\forall z\left(z<y \wedge(z=x \vee x<z) \rightarrow \alpha_{\varphi}(z)\right)\right), \\
& \alpha_{\varphi} \mathbf{U}^{a} \psi(x):=\exists y\left((x<y \vee x=y) \wedge \alpha_{\psi}(y) \wedge \forall u \forall v(u<y \wedge y<v \wedge \mu(u, v) \rightarrow u<x) \wedge\right. \\
& \left.\forall z\left(z<y \wedge(z=x \vee x<z) \wedge \forall u \forall v(u<z \wedge z<v \wedge \mu(u, v) \rightarrow u<x) \rightarrow \alpha_{\varphi}(z)\right)\right), \\
& \alpha_{\varphi \mathbf{S}^{c} \psi}(x):=\alpha_{\psi}(x) \vee \exists y\left(y<x \wedge \alpha_{\text {call }}(y) \wedge \forall z(\mu(y, z) \rightarrow x<z) \wedge \alpha_{\psi}(y) \wedge\right. \\
& \left.\forall z\left(\left((z=x) \vee\left(\alpha_{\text {call }}(z) \wedge z<x \wedge y<z \wedge \forall u(\mu(z, u) \rightarrow x<u)\right)\right) \rightarrow \alpha_{\varphi}(z)\right)\right), \\
& \alpha_{\mathcal{C} \varphi}(x):=\left(\neg \exists y \exists z(\mu(y, z) \wedge y<x \wedge x<z) \wedge \forall z\left(\neg \exists u(u<z) \rightarrow \alpha_{\varphi}(z)^{[z, x]}\right) \vee\right. \\
& (\exists y \exists z(\mu(y, z) \wedge y<x \wedge x<z \wedge \\
& \left.\left.\forall u \forall v(u<x \wedge x<v \wedge \mu(u, v) \rightarrow u=y \vee u<y) \wedge \alpha_{\varphi}(y)^{[y, x]}\right)\right), \\
& \alpha_{\mathcal{R} \varphi}(x):=\left(\neg \exists y \exists z(\mu(y, z) \wedge y<x \wedge x<z) \wedge \forall z\left(\neg \exists u(z<u) \rightarrow \alpha_{\varphi}(x)^{[x, z]}\right) \vee\right. \\
& (\exists y \exists z(\mu(y, z) \wedge y<x \wedge x<z \wedge \\
& \left.\left.\forall u \forall v(u<x \wedge x<v \wedge \mu(u, v) \rightarrow u=y \vee u<y) \wedge \alpha_{\varphi}(x)^{[x, z]}\right)\right) .
\end{aligned}
$$

We now show the other direction, that is, $\mathrm{FO} \subseteq$ CaRet $+\{\mathcal{R}, \mathcal{C}\}$. We start by proving the result for FO sentences (that is, we prove that for every FO sentence $\varphi$ there is an CaRet $+\{\mathcal{R}, \mathcal{C}\}$ formula $\psi$, such that $\bar{w} \models \varphi$ iff $(\bar{w}, 1) \models \psi)$, and then extend it to the case of FO formulas with one free variable. Let $\varphi$ be an FO sentence. We use induction on the quantifier rank to prove that $\varphi$ is equivalent to an CaRet $+\{\mathcal{R}, \mathcal{C}\}$ formula.

For $k=0$ the property trivially holds, as $\varphi$ is a Boolean combination of formulas of the form $P_{a}(\min ), P_{a}(\max ), \min <\max , \mu(\min , \max )$, etc. All of them can be easily expressed in CaRet $+\{\mathcal{R}, \mathcal{C}\}$.

We now prove for $k+1$ assuming that the property holds for $k$. Since every FO sentence of quantifier rank $k+1$ is a Boolean combination of $\mathrm{FO}$ sentences of the form $\exists x \varphi(x)$, where $\varphi(x)$ is a formula of quantifier rank $k$, we just have to show how to express in CaRet $+\{\mathcal{R}, \mathcal{C}\}$ a sentence of this form.

Let $\Gamma$ be the set of all rank- $k$ types of nested words over alphabet $\Sigma \cup\{\min , \max \}$. We distinguish by $\tau_{\varepsilon}$ the rank- $k$ type of the empty nested word. By induction hypothesis, for 
each $\tau \in \Gamma$ there is an CaRet $+\{\mathcal{R}, \mathcal{C}\}$ formula $\xi_{\tau}$ such that $(\bar{w}, 1) \models \xi_{\tau}$ iff the rank- $k$ type of $(\bar{w}, \min , \max )$ is $\tau$.

Let $\Lambda$ be the set of all rank- $(k+2)$ types of words over alphabet $\Gamma \times \Gamma$. We first construct, for each $\lambda \in \Lambda$, an CaRet $+\{\mathcal{R}, \mathcal{C}\}$ formula $\alpha_{\lambda}$ over alphabet $\Sigma$ such that,

$$
(\bar{w}, i) \models \alpha_{\lambda} \quad \Longleftrightarrow \quad \text { the rank- }(k+2) \text { type of } \Omega_{k}(\bar{w}, i) \text { is } \lambda \text {, }
$$

for each nested word $\bar{w}$ and position $i$ of $\bar{w}$.

Fix $\lambda \in \Lambda$. From Kamp's theorem [14, there is an LTL formula $\beta_{\lambda}$ over alphabet $\Gamma \times \Gamma$ such that a word $u$ satisfies $\beta_{\lambda}$ evaluated on its last element iff the rank- $(k+2)$ type of $u$ is $\lambda$. By the separation property of LTL, we can assume that $\beta_{\lambda}$ only mentions past modalities $\ominus$ and $\mathbf{S}$. Moreover, given that $\varphi \mathbf{S} \psi \equiv \psi \vee(\varphi \wedge \ominus(\varphi \mathbf{S} \psi))$, we can also assume that $\beta_{\lambda}$ is a Boolean combination of formulas of the form either $\theta$ or $\ominus \theta^{\prime}$, where $\theta$ does not mention any temporal modality and $\theta^{\prime}$ is an arbitrary past LTL formula. Thus, since CaRet $+\{\mathcal{R}, \mathcal{C}\}$ is closed under Boolean combinations, to show how to define $\alpha_{\lambda}$ from $\beta_{\lambda}$, we only need to consider two cases: (1) $\beta_{\lambda}$ is an LTL formula over $\Gamma \times \Gamma$ without temporal modalities, and (2) $\beta_{\lambda}$ is of the form $\Theta \theta$, where $\theta$ is an arbitrary past LTL formula over $\Gamma \times \Gamma$. Next we consider these two cases.

- Assume that $\beta_{\lambda}$ is an LTL formula without temporal modalities. Then $\alpha_{\lambda}$ is defined to be $\beta_{\lambda}^{\circ}$, where ()$^{\circ}$ is defined recursively as follows. Given $\left(\tau, \tau^{\prime}\right) \in \Gamma \times \Gamma,\left(\tau, \tau^{\prime}\right)^{\circ}$ is defined as follows, where we assume that $\tau_{a}$ is the rank- $k$ type of any nested word with a single element labeled $a(a \in \Sigma)$ :

(1) If $\tau, \tau^{\prime} \neq \tau_{\varepsilon}$, then $\left(\tau, \tau^{\prime}\right)^{\circ}$ is defined as the disjunction of the following formulas:

(a) $\left(\neg\right.$ ret $\wedge \ominus_{c} \top \wedge \ominus\left(\neg\right.$ call $\left.\left.\wedge \mathcal{C} \xi_{\tau}\right) \wedge \mathcal{R} \xi_{\tau^{\prime}}\right)$;

(b) $\bigvee_{\left\{a \mid \tau=\tau_{a}\right\}}\left(\neg\right.$ ret $\left.\wedge \Theta_{c} \top \wedge \ominus(\operatorname{call} \wedge a) \wedge \mathcal{R} \xi_{\tau^{\prime}}\right)$;

(c) $\left(\neg\right.$ ret $\left.\wedge \neg \Theta_{c} \top \wedge \ominus \mathcal{C} \xi_{\tau} \wedge \mathcal{R} \xi_{\tau^{\prime}}\right)$;

(d) $\left(\right.$ pret $\left.\wedge \ominus \mathcal{C} \xi_{\tau} \wedge \mathcal{R} \xi_{\tau^{\prime}}\right)$

(e) $\bigvee_{\left\{a \mid \tau^{\prime}=\tau_{a}\right\}}\left(\right.$ ret $\wedge \neg$ pret $\left.\wedge \mathrm{a} \wedge \ominus\left(\neg \operatorname{call} \wedge \mathcal{C} \xi_{\tau}\right)\right)$;

(f) $\bigvee_{\left\{(a, b) \mid \tau=\tau_{a}, \tau^{\prime}=\tau_{b}\right\}}($ ret $\wedge \neg$ pret $\wedge \mathrm{b} \wedge \ominus(\operatorname{call} \wedge \mathrm{a}))$.

(2) if $\tau^{\prime}=\tau_{\varepsilon}$ then $\left(\tau, \tau^{\prime}\right)^{\circ}$ is simply $\neg \top$; and

(3) if $\tau=\tau_{\varepsilon}$ and $\tau^{\prime} \neq \tau_{\varepsilon}$, then $\left(\tau, \tau^{\prime}\right)^{\circ}$ is defined as $\neg \ominus \top \wedge \mathcal{R} \xi_{\tau^{\prime}}$.

Furthermore, if $\psi$ and $\varphi$ are LTL formulas without temporal modalities, then

$$
\begin{aligned}
(\neg \varphi)^{\circ} & :=\neg \varphi^{\circ}, \\
(\varphi \vee \psi)^{\circ} & :=\varphi^{\circ} \vee \psi^{\circ} .
\end{aligned}
$$

- Assume that $\beta_{\lambda}$ is a formula of the form $\ominus \theta$, where $\theta$ is an arbitrary past LTL formula. Then $\alpha_{\lambda}$ is defined to be $\beta_{\lambda}^{\star}$, where ()$^{\star}$ is defined recursively as follows. Given $\left(\tau, \tau^{\prime}\right) \in$ $\Gamma \times \Gamma,\left(\tau, \tau^{\prime}\right)^{\star}$ is defined as follows:

(1) If $\tau, \tau^{\prime} \neq \tau_{\varepsilon}$, then $\left(\tau, \tau^{\prime}\right)^{\star}$ is defined as the disjunction of the following formulas:

(a) $\ominus\left(\left(\neg\right.\right.$ call $\left.\left.\vee\left(\operatorname{call} \wedge \neg \bigcirc_{\mu} \top\right)\right) \wedge \mathcal{C} \xi_{\tau}\right) \wedge \bigcirc_{\mu} \bigcirc\left((\neg\right.$ ret $\vee$ pret $\left.) \wedge \mathcal{R} \xi_{\tau^{\prime}}\right)$;

(b) $\bigvee_{\left\{a \mid \tau=\tau_{a}\right\}} \odot\left(\operatorname{call} \wedge \bigcirc_{\mu} \top \wedge a\right) \wedge \bigcirc_{\mu} \bigcirc\left(\neg\right.$ ret $\left.\wedge \mathcal{R} \xi_{\tau^{\prime}}\right)$;

(c) $\bigvee_{\left\{(a, b) \mid \tau=\tau_{a}, \tau^{\prime}=\tau_{b}\right\}} \ominus\left(\operatorname{call} \wedge \bigcirc_{\mu} \top \wedge a\right) \wedge \bigcirc_{\mu} \bigcirc($ ret $\wedge b)$;

(d) $\bigvee_{\left\{a \mid \tau^{\prime}=\tau_{a}\right\}} \Theta\left(\neg \operatorname{call} \wedge \mathcal{C} \xi_{\tau}\right) \wedge \bigcirc_{\mu} \bigcirc($ ret $\wedge \neg$ pret $\wedge$ a $)$.

(2) if $\tau=\tau_{e}$ and $\tau^{\prime} \neq \tau_{e}$, then $\left(\tau, \tau^{\prime}\right)^{\star}$ is defined as $\neg \odot \top \wedge \bigcirc_{\mu} \bigcirc \mathcal{R} \xi_{\tau^{\prime}}$;

(3) if $\tau \neq \tau_{\varepsilon}$ and $\tau^{\prime}=\tau_{e}$, then $\left(\tau, \tau^{\prime}\right)^{\star}$ is defined as $\ominus \mathcal{C} \xi_{\tau} \wedge \neg \bigcirc_{\mu} \bigcirc \top$; and

(4) if $\tau, \tau^{\prime}=\tau_{\varepsilon}$ then $\left(\tau, \tau^{\prime}\right)^{\star}$ is defined as $\neg \odot \top \wedge \neg \bigcirc_{\mu} \bigcirc \top$. 
Furthermore, if $\psi$ and $\varphi$ are past LTL formulas, then

$$
\begin{aligned}
(\neg \varphi)^{\star} & :=\neg \varphi^{\star}, \\
(\varphi \vee \psi)^{\star} & :=\varphi^{\star} \vee \psi^{\star}, \\
(\Theta \varphi)^{\star} & :=\Theta_{c} \varphi^{\star}, \\
(\varphi \mathbf{S} \psi)^{\star} & :=\varphi^{\star} \mathbf{S}^{c} \psi^{\star} .
\end{aligned}
$$

Now, let $\exists x \varphi(x)$ be an FO sentence such that the quantifier rank of $\varphi(x)$ is $k$. Then, from our composition method $\varphi(x)$ can be expressed in CaRet $+\{\mathcal{R}, \mathcal{C}\}$ as the formula $\bigvee_{\lambda \in \Lambda^{\prime}} \alpha_{\lambda}$, where $\Lambda^{\prime} \subseteq \Lambda$ is the set of all rank- $(k+2)$ types of words over alphabet $\Gamma \times \Gamma$ that belong to $\left\{\Omega_{k}(\bar{w}, i) \mid \quad \bar{w} \models \varphi(i)\right\}$. Thus, $\exists x \varphi(x)$ can be expressed as the following CaRet $+\{\mathcal{R}, \mathcal{C}\}$ formula: $\top \mathbf{U}\left(\bigvee_{\lambda \in \Lambda^{\prime}} \alpha_{\lambda}\right)$. This concludes the proof of the theorem.

Finally, from the composition method and the previous proof we see that the equivalence $\mathrm{FO}=\mathrm{CaRet}+\{\mathcal{R}, \mathcal{C}\}$ also holds for unary queries over nested words.

\section{Model-Checking And SAtisfiability}

In this section we show that both satisfiability and model-checking are decidable in singleexponential-time for NWTL, and in polynomial time in the size of the model. Here we assume the model of the procedural program is given as a Recursive State Machine (RSM) [1. (Runs of an RSM can naturally be viewed as nested words when matching function calls (or "box entries") and returns (or "box exits") along the run are paired together.) In fact we prove this bound for $\mathrm{NWTL}^{+}$, an FO-complete extension of NWTL with all of $\mathbf{U}, \mathbf{S}, \mathbf{U}^{c}, \mathbf{S}^{c}, \mathbf{U}^{a}, \mathbf{S}^{a}$. We use automata-theoretic techniques: translating formulae into equivalent automata on nested words. We then show that the logic based on adding the within operator to $\mathrm{NWTL}^{+}$, (and even just adding within to CaRet) requires doubly-exponential time for model-checking, but is exponentially more succinct.

5.1. Nested word automata. A nondeterministic Büchi nested word automaton (BNWA) $A$ over an alphabet $\Sigma$ is a structure $\left(Q, Q_{0}, Q_{f}, P, P_{0}, P_{f}, \delta_{c}, \delta_{i}, \delta_{r}\right)$ consisting of a finite set of states $Q$, a set of initial states $Q_{0} \subseteq Q$, a set of Büchi accepting states $Q_{f} \subseteq Q$, a set of hierarchical symbols $P$, a set of initial hierarchical symbols $P_{0} \subseteq P$, a set of final hierarchical symbols $P_{f} \subseteq P$, a call-transition relation $\delta_{c} \subseteq Q \times \Sigma \times Q \times P$, an internaltransition relation $\delta_{i} \subseteq Q \times \Sigma \times Q$, and a return-transition relation $\delta_{r} \subseteq Q \times P \times \Sigma \times Q$. The automaton $A$ starts in an initial state and reads the nested word from left to right. The state is propagated along the linear edges as in the case of a standard word automaton. However, at a call, the nested word automaton propagates state along the linear edge as well as a hierarchical symbol along the nesting edge (if there is no matching return, then the latter is required to be in $P_{f}$ for acceptance). At a matched return, the new state is determined based on the state propagated along the linear edge as well as the symbol along the incoming nesting edge (edges incident upon unmatched returns are assumed to be labeled with initial hierarchical symbols).

Formally, a run $r$ of the automaton $A$ over a nested word $\bar{w}=\left(a_{1} a_{2} \ldots, \mu\right.$, call, ret $)$ is a sequence $q_{0}, q_{1}, \ldots$ of states along the linear edges, and a sequence $p_{i}$, for every call position $i$, of hierarchical symbols along nesting edges, such that $q_{0} \in Q_{0}$ and for each position $i$, if $i$ is a call then $\left(q_{i-1}, a_{i}, q_{i}, p_{i}\right) \in \delta_{c}$; if $i$ is internal, then $\left(q_{i-1}, a_{i}, q_{i}\right) \in \delta_{i}$; if $i$ is a return such that $\mu(j, i)$, then $\left(q_{i-1}, p_{j}, a_{i}, q_{i}\right) \in \delta_{r}$; and if $i$ is an unmatched return then 
$\left(q_{i-1}, p, a_{i}, q_{i}\right) \in \delta_{r}$ for some $p \in P_{0}$. The run $r$ is accepting if (1) for all pending calls $i$, $p_{i} \in P_{f}$, and (2) the final state $q_{\ell} \in Q_{f}$ if $\bar{w}$ is a finite word of length $\ell$, and for infinitely many positions $i, q_{i} \in Q_{f}$, if $\bar{w}$ is a nested $\omega$-word. The automaton $A$ accepts the nested word $\bar{w}$ if it has an accepting run over $\bar{w}$.

Nested word automata have the same expressiveness as the monadic second order logic over nested words, and the language emptiness problem for them can be decided in polynomial-time [4.

5.2. Tableau construction. We now show how to build a BNWA accepting the satisfying models of a formula of $\mathrm{NWTL}^{+}$. This leads to decision procedures for satisfiability and model checking.

Given a formula $\varphi$, we wish to construct a Büchi nested word automaton $A_{\varphi}$ whose states correspond to sets of subformulas of $\varphi$. Intuitively, given a nested word $\bar{w}$, a run $r$, which is a linear sequence $q_{0} q_{1} \ldots$ of states and symbols $p_{i}$ labeling nesting edges from call positions, should be such that each state $q_{i}$ is precisely the set of formulas that hold at position $i+1$. The label $p_{i}$ is used to remember abstract-next formulas that hold at position $i$ and the abstract-previous formulas that hold at matching return. For clarity of presentation, we first focus on formulas with next operators $\bigcirc$ and $\bigcirc_{\mu}$, and until over summary-down paths.

Given a formula $\varphi$, the closure of $\varphi$, denoted by $\operatorname{cl}(\varphi)$, is the smallest set that satisfies the following properties:

- $\operatorname{cl}(\varphi)$ contains $\varphi, \operatorname{call}$, ret, int, and Oret;

- if either $\neg \psi$, or $\bigcirc \psi$ or $\bigcirc_{\mu} \psi$ is in $\operatorname{cl}(\varphi)$ then $\psi \in \operatorname{cl}(\varphi)$;

- if $\psi \vee \psi^{\prime} \in \operatorname{cl}(\varphi)$, then $\psi, \psi^{\prime} \in \operatorname{cl}(\varphi)$;

- if $\psi \mathbf{U}^{\sigma \downarrow} \psi^{\prime} \in \operatorname{cl}(\varphi)$, then $\psi, \psi^{\prime}, \bigcirc\left(\psi \mathbf{U}^{\sigma \downarrow} \psi^{\prime}\right)$, and $\bigcirc_{\mu}\left(\psi \mathbf{U}^{\sigma \downarrow} \psi^{\prime}\right)$ are in $c l(\varphi)$; and

- if $\psi \in \operatorname{cl}(\varphi)$ and $\psi$ is not of the form $\neg \theta$ (for any $\theta$ ), then $\neg \psi \in \operatorname{cl}(\varphi)$.

It is straightforward to see that the size of $\operatorname{cl}(\varphi)$ is only linear in the size of $\varphi$. Henceforth, we identify $\neg \neg \psi$ with the formula $\psi$.

An atom of $\varphi$ is a set $\Phi \subseteq \operatorname{cl}(\varphi)$ that satisfies the following properties:

- For every $\psi \in \operatorname{cl}(\varphi), \psi \in \Phi$ iff $\neg \psi \notin \Phi$.

- For every formula $\psi \vee \psi^{\prime} \in \operatorname{cl}(\varphi), \psi \vee \psi^{\prime} \in \Phi$ iff $\left(\psi \in \Phi\right.$ or $\left.\psi^{\prime} \in \Phi\right)$.

- For every formula $\psi \mathbf{U}^{\sigma \downarrow} \psi^{\prime} \in \operatorname{cl}(\varphi), \psi \mathbf{U}^{\sigma \downarrow} \psi^{\prime} \in \Phi$ iff either $\psi^{\prime} \in \Phi$ or $(\psi \in \Phi$ and Oret $\notin \Phi$ and $\left.\bigcirc\left(\psi \mathbf{U}^{\sigma \downarrow} \psi^{\prime}\right) \in \Phi\right)$ or $\left(\psi \in \Phi\right.$ and $\left.\bigcirc_{\mu}\left(\psi \mathbf{U}^{\sigma \downarrow} \psi^{\prime}\right) \in \Phi\right)$.

- $\Phi$ contains exactly one of the elements in the set $\{$ call, ret, int $\}$.

- If $\bigcirc_{\mu} \psi \in \Phi$ for some $\psi$, then call $\in \Phi$.

These clauses capture local consistency requirements. In particular, a summary-down until formula $\psi \mathbf{U}^{\sigma \downarrow} \psi^{\prime}$ holds at a position if either the second argument $\psi^{\prime}$ holds now, or $\psi$ holds now and satisfaction of $\psi \mathbf{U}^{\sigma \downarrow} \psi^{\prime}$ is propagated along a call, internal, or nesting edge.

A hierarchical-atom of $\varphi$ is a set $\Phi \subseteq \operatorname{cl}(\varphi)$ such that if $\psi \in \Phi$ then $\bigcirc_{\mu} \psi \in \operatorname{cl}(\varphi)$. A hierarchical-atom contains possible abstract-next obligations to be propagated across nesting edges.

Given a formula $\varphi$, we build a nested word automaton $A_{\varphi}$ as follows. The alphabet $\Sigma$ is $2^{A P}$, where $A P$ is the set of atomic propositions.

(1) Atoms of $\varphi$ are states of $A_{\varphi}$;

(2) An atom $\Phi$ is an initial state iff $\varphi \in \Phi$;

(3) Hierarchical-atoms of $\varphi$ are hierarchical symbols of $A_{\varphi}$; 
(4) All hierarchical symbols are initial;

(5) For atoms $\Phi, \Psi$ and a symbol $a \subseteq A P,(\Phi, a, \Psi)$ is an internal transition of $A_{\varphi}$ iff (a) int $\in \Phi$; and (b) for $p \in A P, p \in a$ iff $p \in \Phi$; and (c) for each $\bigcirc \psi \in \operatorname{cl}(\varphi), \psi \in \Psi$ iff $\bigcirc \psi \in \Phi$.

(6) For atoms $\Phi, \Psi_{l}$, a hierarchical-atom $\Psi_{h}$, and a symbol $a \subseteq A P,\left(\Phi, a, \Psi_{l}, \Psi_{h}\right)$ is a call transition of $A_{\varphi}$ iff (a) call $\in \Phi$; and (b) for $p \in A P, p \in a$ iff $p \in \Phi$; and (c) for each $\bigcirc \psi \in \operatorname{cl}(\varphi), \psi \in \Psi_{l}$ iff $\bigcirc \psi \in \Phi$; and (d) for each $\bigcirc_{\mu} \psi \in \operatorname{cl}(\varphi), \psi \in \Psi_{h}$ iff $\bigcirc_{\mu} \psi \in \Phi$.

(7) For atoms $\Phi_{l}, \Psi$, hierarchical-atom $\Psi_{h}$, and a symbol $a \subseteq A P,\left(\Phi_{l}, \Phi_{h}, a, \Psi\right)$ is a return transition of $A_{\varphi}$ iff (a) ret $\in \Phi_{l}$; and (b) for $p \in A P, p \in a$ iff $p \in \Phi_{l}$; and (c) for each $\bigcirc \psi \in \operatorname{cl}(\varphi), \psi \in \Psi$ iff $\bigcirc \psi \in \Phi_{l}$; and (d) for each $\bigcirc_{\mu} \psi \in \operatorname{cl}(\varphi), \psi \in \Phi_{h}$ iff $\psi \in \Phi_{l}$.

The transition relation ensures that the current symbol is consistent with the atomic propositions in the current state, and next operators requirements are correctly propagated.

The sole final hierarchical symbol is the empty hierarchical-atom. This ensures that, in an accepting run, at a pending call, no requirements are propagated along the nesting edge. For each until-formula $\psi$ in the closure, let $F_{\psi}$ be the set of atoms that either do not contain $\psi$ or contain the second argument of $\psi$. Then a nested word $\bar{w}$ over the alphabet $2^{A P}$ satisfies $\varphi$ iff there is a run $r$ of $A_{\varphi}$ over $\bar{w}$ such that all pending call edges are labeled with the sole final hierarchical symbol, and for each until-formula $\psi \in \operatorname{cl}(\varphi)$, for infinitely many positions $i, q_{i} \in F_{\psi}$. This multi-Büchi accepting condition can be translated to Büchi acceptance as usual by adding a counter.

Now we proceed to show how to handle various forms of until operators. In each case, we specify the changes needed to the definition of the closure and local consistency requirements for atoms.

Global paths: If $\psi \mathbf{U} \psi^{\prime} \in \operatorname{cl}(\varphi)$, then $\psi, \psi^{\prime}, \bigcirc\left(\psi \mathbf{U} \psi^{\prime}\right)$ are in $\operatorname{cl}(\varphi)$. Local consistency of $\Phi$ requires that for every formula $\psi \mathbf{U} \psi^{\prime} \in \operatorname{cl}(\varphi), \psi \mathbf{U} \psi^{\prime} \in \Phi$ iff either $\psi^{\prime} \in \Phi$ or $(\psi \in \Phi$ and $\left.\bigcirc\left(\psi \mathbf{U} \psi^{\prime}\right) \in \Phi\right)$.

Summary-up paths: If $\psi \mathbf{U}^{\sigma \uparrow} \psi^{\prime} \in \operatorname{cl}(\varphi)$, then $\psi, \psi^{\prime}, \bigcirc\left(\psi \mathbf{U}^{\sigma \uparrow} \psi^{\prime}\right)$, and $\bigcirc_{\mu}\left(\psi \mathbf{U}^{\sigma \uparrow} \psi^{\prime}\right)$ are in $\operatorname{cl}(\varphi)$. Local consistency of $\Phi$ requires that for every formula $\psi \mathbf{U}^{\sigma \uparrow} \psi^{\prime} \in \operatorname{cl}(\varphi)$, $\psi \mathbf{U}^{\sigma \uparrow} \psi^{\prime} \in \Phi$ iff either $\psi^{\prime} \in \Phi$, or $\left(\psi \in \Phi\right.$ and call $\in \Phi$ and $\left.\bigcirc_{\mu}\left(\psi \mathbf{U}^{\sigma \uparrow} \psi^{\prime}\right) \in \Phi\right)$, or $\left(\psi \in \Phi\right.$ and call $\notin \Phi$ and $\left.\bigcirc\left(\psi \mathbf{U}^{\sigma \uparrow} \psi^{\prime}\right) \in \Phi\right)$.

Abstract paths: If $\psi \mathbf{U}^{a} \psi^{\prime} \in \operatorname{cl}(\varphi)$, then $\psi, \psi^{\prime}, \bigcirc\left(\psi \mathbf{U}^{a} \psi^{\prime}\right), \bigcirc \bigodot_{\mu} \top$, and $\bigcirc_{\mu}\left(\psi \mathbf{U}^{a} \psi^{\prime}\right)$ are in $\operatorname{cl}(\varphi)$. Local consistency of $\Phi$ requires that for every formula $\psi \mathbf{U}^{a} \psi^{\prime} \in \operatorname{cl}(\varphi)$, $\psi \mathbf{U}^{a} \psi^{\prime} \in \Phi$ iff either $\psi^{\prime} \in \Phi$, or $\left(\psi \in \Phi\right.$ and call $\in \Phi$ and $\left.\bigcirc_{\mu}\left(\psi \mathbf{U}^{a} \psi^{\prime}\right) \in \Phi\right)$, or $\left(\psi \in \Phi\right.$ and call $\notin \Phi$ and $\bigcirc$ ret $\notin \Phi$ and $\left.\bigcirc\left(\psi \mathbf{U}^{a} \psi^{\prime}\right) \in \Phi\right)$, or $(\psi \in \Phi$ and Oret $\in \Phi$ and $\bigcirc \Theta_{\mu} \top \notin \Phi$ and $\left.\bigcirc\left(\psi \mathbf{U}^{a} \psi^{\prime}\right) \in \Phi\right)$. The last case accounts for propagation of the eventuality across unmatched returns.

Call paths: Recall that positions along a call path are related by the innermost call operator: a call path jumps from a call position $i$ to a position $j$ such that $i=\mathcal{C}(j)$. Thus, a call path can be simulated by a summary-down path consisting of call edges, summary edges and internal edges, where the formula is asserted only before following the call edge. This effect is captured by using an auxiliary operator as follows. If $\psi \mathbf{U}^{c} \psi^{\prime} \in \operatorname{cl}(\varphi)$, then $\psi, \psi^{\prime}, \psi \mathbf{U}^{c^{\prime}} \psi^{\prime}, \bigcirc\left(\psi \mathbf{U}^{c^{\prime}} \psi^{\prime}\right)$, and $\bigcirc_{\mu}\left(\psi \mathbf{U}^{c^{\prime}} \psi^{\prime}\right)$ are in $\operatorname{cl}(\varphi)$. Local consistency of $\Phi$ requires that for every formula $\psi \mathbf{U}^{c} \psi^{\prime} \in c l(\varphi), \psi \mathbf{U}^{c} \psi^{\prime} \in \Phi$ iff either $\psi^{\prime} \in \Phi$ or $\left(\psi \in \Phi\right.$ and call $\in \Phi$ and $\left.\bigcirc\left(\psi \mathbf{U}^{c^{\prime}} \psi^{\prime}\right) \in \Phi\right)$; and $\psi \mathbf{U}^{c^{\prime}} \psi^{\prime} \in \Phi$ iff either $\psi \mathbf{U}^{c} \psi^{\prime} \in \Phi$, or $\bigcirc_{\mu}\left(\psi \mathbf{U}^{c^{\prime}} \psi^{\prime}\right) \in \Phi$, or $\left(\bigcirc\right.$ ret $\notin \Phi$ and $\left.\bigcirc\left(\psi \mathbf{U}^{c^{\prime}} \psi^{\prime}\right) \in \Phi\right)$. 
Summary paths: The summary-until is handled using the fact that $\psi \mathbf{U}^{\sigma} \psi^{\prime}$ is equivalent to $\psi \mathbf{U}^{\sigma \uparrow}\left(\psi \mathbf{U}^{\sigma \downarrow} \psi^{\prime}\right)$.

Note that the definition of $A_{\varphi}$ stays unchanged, as the correct propagation of requirements is handled by next and abstract-next formulas ensured by local consistency. The eventual satisfaction of until formulas is handled the same way as before: for each until-formula $\psi$ in the closure, let $F_{\psi}$ be the set of atoms that either do not contain $\psi$ or contain the second argument of $\psi$, and it is required that each such $F_{\psi}$ is visited infinitely often.

The past-time formulas (previous, abstract-previous, and various forms of since operators) are handled in a symmetric manner. Thus, we have shown:

Theorem 5.1. For a formula $\varphi$ of $\mathrm{NWTL}^{+}$, one can effectively construct a nondeterministic Büchi nested word automaton $A_{\varphi}$ of size $2^{O(|\varphi|)}$ accepting the models of $\varphi$.

Since the automaton $A_{\varphi}$ is exponential in the size of $\varphi$, we can check satisfiability of $\varphi$ in exponential-time by testing emptiness of $A_{\varphi}$. Exptime-hardness follows from the corresponding hardness result for CaRet.

Corollary 5.2. The satisfiability problem for $\mathrm{NWTL}^{+}$is EXPTIME-complete.

When programs are modeled by nested word automata $A$ (or equivalently, pushdown automata, or recursive state machines), and specifications are given by formulas $\varphi$ of $\mathrm{NWTL}^{+}$, we can use the classical automata-theoretic approach: negate the specification, build the NWA $A_{\neg \varphi}$ accepting models that violate $\varphi$, take the product with the program $A$, and test for emptiness of $L(A) \cap L\left(A_{\neg \varphi}\right)$. Note that the program typically will be given more compactly, say, as a Boolean program [5], and thus, the NWA $A$ may itself be exponential in the size of the input.

Corollary 5.3. Model checking $\mathrm{NWTL}^{+}$specifications with respect to Boolean programs is EXPTIME-complete. If the program model is given as a recursive state machine or nested word automaton, the running time is polynomial in the model and exponential in the $\mathrm{NWTL}^{+}$formula, and remains ExPTIME-complete.

5.3. Checking the within operator. We now show that adding within operators makes model-checking doubly exponential. Given a formula $\varphi$ of NWTL or $\mathrm{NWTL}^{+}$, let $p_{\varphi}$ be a special proposition that does not appear in $\varphi$. Let $W_{\varphi}$ be the language of nested words $\bar{w}$ such that for each position $i,(\bar{w}, i) \models p_{\varphi}$ iff $(\bar{w}, i) \models \mathcal{W} \varphi$. We construct a doublyexponential automaton $B$ that captures $W_{\varphi}$. First, using the tableau construction for $\mathrm{NWTL}^{+}$, we construct an exponential-size automaton $A$ that captures nested words that satisfy $\varphi$. Intuitively, every time a proposition $p_{\varphi}$ is encountered, we want to start a new copy of $A$, and a state of $B$ keeps track of states of multiple copies of $A$. At a call, $B$ guesses whether the call has a matching return or not. In the latter case, as in case of determinization construction for nested word automata [4], we need to maintain pairs of states of $A$ so that the join at return positions can be done correctly. A state of $B$, then, is either a set of states of $A$ or a set of pairs of states of $A$. We explain the latter case. The intended meaning is that a pair $\left(q, q^{\prime}\right)$ belongs to the state of $B$, while reading position $i$ of a nested word $\bar{w}$, if the subword from $i$ to the first unmatched return can take the automaton $A$ from state $q$ to state $q^{\prime}$. When reading an internal symbol $a$, a summary $\left(q, q^{\prime}\right)$ in the current state can be updated to $\left(u, q^{\prime}\right)$, provided $A$ has an internal transition from $q$ to $u$ on symbol $a$. Let $B$ read a call symbol $a$. Consider a summary $\left(q, q^{\prime}\right)$ in the current state, and 
a call-transition $\left(q, a, q_{l}, q_{h}\right)$ of $A$. Then $B$ guesses the return transition $\left(u_{l}, q_{h}, b, u\right)$ that will be used by $A$ at the matching return, and sends the summary $\left(q_{l}, u_{l}\right)$ along the call edge and the triple $\left(b, u, q^{\prime}\right)$ along the nesting edge. While processing a return symbol $b$, the current state of $B$ must contain summaries only of the form $(q, q)$ where the two states match, and for each summary $\left(b, u, q^{\prime}\right)$ retrieved from the state along the nesting edge, the new state contains $\left(u, q^{\prime}\right)$. Finally, $B$ must enforce that $\mathcal{W} \varphi$ holds when $p_{\varphi}$ is read. Only a call symbol $a$ can contain the proposition $p_{\varphi}$, and when reading such a symbol, $B$ guesses a call transition $\left(q_{0}, a, q_{l}, q_{h}\right)$, where $q_{0}$ is the initial state of $A$, and a return transition $\left(u_{l}, q_{h}, b, q_{f}\right)$, where $q_{f}$ is an accepting state of $A$, and sends the summary $\left(q_{l}, u_{l}\right)$ along the call edge and the symbol $b$ along the nesting edge.

Lemma 5.4. For every formula $\varphi$ of $\mathrm{NWTL}^{+}$, there is a nested word automaton that accepts the language $W_{\varphi}$ and has size doubly-exponential in $|\varphi|$.

Consider a formula $\varphi$ of $\mathrm{NWTL}^{+}+\mathcal{W}$. For every within-subformula $\mathcal{W} \varrho$ of $\varphi$, let $\varrho^{\prime}$ be obtained from $\varrho$ by substituting each top-level subformula $\mathcal{W} \psi$ in $\varrho$ by the proposition $p_{\psi}$. Each of these primed formulas is a formula of $\mathrm{NWTL}^{+}$. Then, if we take the product of the nested word automata accepting $W_{\varrho^{\prime}}$ corresponding to all the within-subformulas $\varrho$, together with the nested word automaton $A_{\varphi^{\prime}}$, the resulting language captures the set of models of $\varphi$. Intuitively, the automaton for $W_{\varrho^{\prime}}$ is ensuring that the truth of the proposition $p_{\varrho}$ reflects the truth of the subformula $\mathcal{W} \varrho$. If $\varrho$ itself has a within-subformula $\mathcal{W} \psi$, then the automaton for $\varrho$ treats it as an atomic proposition $p_{\psi}$, and the automaton checking $p_{\psi}$, running in parallel, makes sure that the truth of $p_{\psi}$ correctly reflects the truth of $\mathcal{W} \psi$.

For the lower bound, the decision problem for LTL games can be reduced to the satisfiability problem for formulas with linear untils and within operators [18, and this shows that for CaRet extended with the within operator, the satisfiability problem is 2ExPTIME-hard. We thus obtain:

Theorem 5.5. For the logic $\mathrm{NWTL}^{+}$extended with the within operator $\mathcal{W}$ the satisfiability problem and the model checking problem with respect to Boolean programs, are both 2EXPTIME-complete.

Remark: checking $\bar{w} \models \varphi$ for finite nested words. For finite nested words, one evaluates the complexity of checking whether the given word satisfies a formula, in terms of the length $|\bar{w}|$ of the word and the size of the formula. A straightforward recursion on subformulas shows that for NWTL formulas the complexity of this check is $O(|\bar{w}| \cdot|\varphi|)$, and for both logics with within operators, CaRet $+\{\mathcal{C}, \mathcal{R}\}$ and $\operatorname{LTL}^{\mu}+\mathcal{W}$, it is $O\left(|\bar{w}|^{2} \cdot|\varphi|\right)$.

5.4. On within and succinctness. We saw that adding within operators to $\mathrm{NWTL}^{+}$ increases the complexity of model-checking by one exponent. Thus there is no polynomialtime translation from $\mathrm{NWTL}^{+}+\mathcal{W}$ to $\mathrm{NWTL}^{+}$. We now prove a stronger result that gives a space bound as well: while $\mathrm{NWTL}^{+}+\mathcal{W}$ has the same power as $\mathrm{NWTL}^{+}$, its formulae can be exponentially more succinct than formulas of $\mathrm{NWTL}^{+}$. That is, there is a sequence $\varphi_{n}, n \in \mathbb{N}$, of $\mathrm{NWTL}^{+}+\mathcal{W}$ formulas such that $\varphi_{n}$ is of size $O(n)$, and the smallest formula of $\mathrm{NWTL}^{+}$equivalent to $\varphi_{n}$ is of size $2^{\Omega(n)}$. For this result, we require nested $\omega$-words to be over the alphabet $2^{A P}$.

Theorem 5.6. $\mathrm{NWTL}^{+}+\mathcal{W}$ is exponentially more succinct than $\mathrm{NWTL}^{+}$. 
Proof. The proof is based upon succinctness results in [9, 23, by adapting their examples to nested words.

From the FO completeness of $\mathrm{NWTL}^{+}$, we have that $\mathrm{NWTL}^{+}+\mathcal{W}$ can be translated into $\mathrm{NWTL}^{+}$. We show that at least an exponential blow-up is necessary for such translation. More precisely, we construct a sequence $\left\{\varphi_{n}\right\}_{n \geq 1}$ of $\mathrm{NWTL}^{+}+\mathcal{W}$ formulas of size $O(n)$, such that the shortest $\mathrm{NWTL}^{+}$formula that is equivalent to $\varphi_{n}$ is of size $2^{\Omega(n)}$. Our proof is a modification of similar proofs given in [9, 23]. Assume $\Sigma=\left\{a_{0}, \ldots, a_{n}\right\}$, and let $\varphi_{n}$ be the following $\mathrm{NWTL}^{+}+\mathcal{W}$ formula (here, $\square^{\sigma} \theta$ and $\diamond^{\sigma} \theta$ are abbreviations for $\neg\left(\top \mathbf{U}^{\sigma} \neg \theta\right)$ and $\top \mathbf{S}^{\sigma} \theta$, respectively):

$$
\square^{\sigma}\left(\operatorname{call} \rightarrow \mathcal{W} \square^{\sigma}\left(\left(\bigwedge_{i=1}^{n}\left(a_{i} \leftrightarrow \ominus^{\sigma}\left(a_{i} \wedge \neg \Theta \top\right)\right)\right) \rightarrow\left(a_{0} \leftrightarrow \ominus^{\sigma}\left(a_{0} \wedge \neg \Theta \top\right)\right)\right)\right) .
$$

It is not hard to see that $\bar{w} \models \varphi_{n}$ iff for all positions $i, j$ in $\bar{w}$ such that $\mu(i, j)$ holds, if position $\ell$ in $\bar{w}[i, j]$ coincides with $i$ on $a_{1}, \ldots, a_{n}$, then $\ell$ also coincides with $i$ on $a_{0}$.

It is shown in Theorem 5.1 that for each $\mathrm{NWTL}^{+}$formula $\alpha$, the language

$$
L_{\alpha}=\{\bar{w} \mid \bar{w} \text { is a nested } \omega \text {-word such that } \bar{w} \models \alpha\}
$$

is recognized by a nondeterministic nested word automaton of size $2^{O(|\alpha|)}$. Thus, to prove the theorem, it is enough to show that every such automaton for $L_{\varphi_{n}}$ is of size $2^{2^{\Omega(n)}}$. Let $A$ be a nondeterministic nested word automaton for $L_{\varphi_{n}}$. Assume that $b_{0}, \ldots, b_{2^{n}-1}$ is an enumeration of the symbols in $2^{\Sigma \backslash\left\{a_{0}\right\}}$. For every $K \subseteq\left\{0, \ldots, 2^{n}-1\right\}$ let $\bar{w}_{K}$ be the word $c_{0} \cdots c_{2^{n}-1}$ over alphabet $2^{\Sigma}$, where for each $i \leq 2^{n}-1$ :

$$
c_{i}= \begin{cases}b_{i} & i \in K \\ b_{i} \cup\left\{a_{0}\right\} & \text { otherwise }\end{cases}
$$

It is not hard to see that for each $K \subseteq\left\{0, \ldots, 2^{n}-1\right\}$, the nested $\omega$-word $\left(\bar{w}_{K}^{\omega}, \mu\right)$, where $\mu=\left\{\left(j, 3 \cdot 2^{n}-j+1\right) \mid 1 \leq j \leq 2^{n}\right\}$, is such that $\left(\bar{w}_{K}^{\omega}, \mu\right) \models \varphi_{n}$. Let $\left(q_{K}^{1}, p_{K}^{1}, q_{K}^{2}\right)$ and $\left(q_{K^{\prime}}^{1}, p_{K^{\prime}}^{1}, q_{K^{\prime}}^{2}\right)$ be pairs of states such that (1) there exists an accepting run of $A$ on $\left(\bar{w}_{K}^{\omega}, \mu\right)$ such that $A$ is in state $q_{K}^{1}$ and has hierarchical symbol $p_{K}^{1}$ in call position $2^{n}$, and $A$ is in state $q_{K}^{2}$ in internal position $2 \cdot 2^{n} ;(2)$ there exists an accepting run of $A$ on $\left(\bar{w}_{K^{\prime}}^{\omega}, \mu\right)$ such that $A$ is in state $q_{K^{\prime}}^{1}$ and has hierarchical symbol $p_{K^{\prime}}^{1}$ in call position $2^{n}$, and $A$ is in state $q_{K^{\prime}}^{2}$ in internal position $2 \cdot 2^{n}$. Next we show that $\left(q_{K}^{1}, p_{K}^{1}, q_{K}^{2}\right) \neq\left(q_{K^{\prime}}^{1}, p_{K^{\prime}}^{1}, q_{K^{\prime}}^{2}\right)$ if $K \neq K^{\prime}$. On the contrary, assume that $\left(q_{K}^{1}, p_{K}^{1}, q_{K}^{2}\right)=\left(q_{K^{\prime}}^{1}, p_{K^{\prime}}^{1}, q_{K^{\prime}}^{2}\right)$. Then $A$ accepts $\left(\bar{w}_{K} \bar{w}_{K^{\prime}} \bar{w}_{K}^{\omega}, \mu\right)$, which leads to a contradiction since $\left(\bar{w}_{K} \bar{w}_{K^{\prime}} \bar{w}_{K}^{\omega}, \mu\right) \not \varphi_{n}$. Given that the number of different $K$ 's is $2^{2^{n}}$, the latter implies that the number of different triples of states and hierarchical symbols of $A$ is at least $2^{2^{n}}$. Thus, if $m$ is equal to the number of states of $A$ plus the number of hierarchical symbols of $A$, then $m^{3} \geq 2^{2^{n}}$ and, hence, $m \geq 2^{2^{n-2}}$. Therefore, the size of $A$ is $2^{2^{\Omega(n)}}$. This concludes the proof of the theorem.

\section{Finite-Variable Fragments}

We have already seen that FO formulas in one free variable over nested words can be written using just three distinct variables, as in the case of the usual, unnested, words. For finite nested words this is a consequence of a tree representation of nested words and the three-variable property for FO over finite trees [20], and for infinite nested words this is a consequence Theorem 4.1. 
In this section we prove two results. First, we give a model-theoretic proof that FO formulas with zero, one, or two free variables over nested words (finite or infinite) are equivalent to $\mathrm{FO}^{3}$ formulas. Given the $\mathrm{FO}=\mathrm{FO}^{3}$ collapse, we ask whether there is a temporal logic expressively complete for $\mathrm{FO}^{2}$, the two-variable fragment. We adapt techniques from [9] to find a temporal logic that has the same expressiveness as $\mathrm{FO}^{2}$ over nested words (in a vocabulary that has successor relations corresponding to the "next" temporal operators).

6.1. The three-variable property. We give a model-theoretic, rather than a syntactic, argument, that uses Ehrenfeucht-Fraïssé games and shows that over nested words, formulas with at most two free variables are equivalent to $\mathrm{FO}^{3}$ formulas. Note that for finite nested words, the translation into trees, already used in the proof of Theorem 4.1, can be done using at most three variables. This means that the result of [20] establishing the 3 -variable property for finite ordered unranked trees gives us the 3-variable property for finite nested words. We prove that $\mathrm{FO}=\mathrm{FO}^{3}$ over arbitrary nested words.

Theorem 6.1. Over finite or infinite nested words, every FO formula with at most 2 free variables is equivalent to an $\mathrm{FO}^{3}$ formula.

Proof. As we mentioned already, in the finite case this is a direct consequence of [20] so we concentrate on the infinite case. It is more convenient for us to prove the result for ordered unranked forests in which a subtree rooted at every node is finite. The way to translate a nested $\omega$-word into such a forest is as follows: when a matched call $i$ with $\mu(i, j)$ is encountered, it defines a subtree with $i$ as its root, and $j+1$ as the next sibling (note that this is different from the translation into binary trees we used before). If $i$ is an internal position, or a pending call or a pending return position, then it has no descendants and its next sibling is $i+1$. Matched returns do not have next sibling, nor do they have any descendants. The nodes in the forest are labeled with call, ret, and the propositions in $\Sigma$, as in the original nested word.

It is routine to define, in FO, relations $\preceq_{\text {desc }}$ and $\preceq_{\text {sib }}$ for descendant and younger sibling in such a forest. Furthermore, from these relations, we can define the usual $\leq$ and $\mu$ in nested words using at most 3 variables as follows. For $x \leq y$, the definition is given by

$$
(y \preceq \text { desc } x) \vee \exists z\left(x \preceq \text { desc } z \wedge \exists x\left(z \prec_{\text {sib }} z \wedge y \preceq \text { desc } x\right)\right)
$$

and for $\mu(x, y)$, by

$$
\left(y \preceq_{\text {desc }} x\right) \wedge \forall z((z \preceq \text { desc } x) \rightarrow \exists x(x=z \wedge x \leq y)) .
$$

Thus, it suffices to prove the three-variable property for such ordered forests, which will be referred to as $\mathcal{A}, \mathcal{B}$, etc. We shall use pebble games. Let $\mathbf{G}_{m}^{v}\left(\mathcal{A}, a_{1}, b_{1}, \mathcal{B}, b_{1}, b_{2}\right)$ be the $m$-move, $v$-pebble game on structures $\mathcal{A}$ and $\mathcal{B}$ where initially pebbles $x_{i}$ are placed on $a_{i}$ in $\mathcal{A}$ and $b_{i}$ in $\mathcal{B}$. Player II has a winning strategy for $\mathbf{G}_{m}^{v}\left(\mathcal{A}, a_{1}, b_{1}, \mathcal{B}, b_{1}, b_{2}\right)$ iff $\mathcal{A}, a_{1}, a_{2}$ and $\mathcal{B}, b_{1}, b_{2}$ agree on all formulas with at most $v$ variables and quantifier-depth $m$. We know from [13] that to prove Theorem 6.1, it suffices to show the following,

Claim 6.2. For all $k$, if Player II has a winning strategy for the game $\mathbf{G}_{3 k+2}^{3}\left(\mathcal{A}, a_{1}, a_{2}\right.$; $\left.\mathcal{B}, b_{1}, b_{2}\right)$, then she also has a winning strategy for the game $\mathbf{G}_{k}^{k}\left(\mathcal{A}, a_{1}, a_{2} ; \mathcal{B}, b_{1}, b_{2}\right)$.

We will show how Player II can win the $k$-pebble game by maintaining a set of 3 -pebble sub-games on which she will copy Player I's moves and decide on good responses using her winning strategy for these smaller 3-pebble games. The choice of these sub-games will 
partition the universe $|\mathcal{A}| \cup|\mathcal{B}|$ so that each play by Player I in the $k$-pebble game will be answered in one 3-pebble game. This is similar to the proof that linear orderings have the 3 -variable property [13.

The subgames, $\mathbf{G}_{m}^{3}\left(\mathcal{A}, a_{1}, a_{2} ; \mathcal{B}, b_{1}, b_{2}\right)$, that Player II maintains will all be vertical in which $a_{2} \preceq_{\text {desc }} a_{1}$ and $b_{2} \preceq_{\text {desc }} b_{1}$ hold, or horizontal in which $a_{1} \prec_{\text {sib }} a_{2}$ and $b_{1} \prec_{\text {sib }} b_{2}$ hold.

The following lemma gives the beginning strategy of Player II in which she replaces an arbitrary game configuration with a set of configurations each of which is vertical or horizontal.

Lemma 6.3. If Player II wins $\mathbf{G}_{m+4}^{3}\left(\mathcal{A}, a_{1}, a_{2} ; \mathcal{B}, b_{1}, b_{2}\right)$. Then there are points $a_{1}^{\prime}, a_{2}^{\prime}$ from $\mathcal{A}$ and $b_{1}^{\prime}, b_{2}^{\prime}$ from $\mathcal{B}$ such that Player II wins the horizontal game $\mathbf{G}_{m+2}^{3}\left(\mathcal{A}, a_{1}^{\prime}, a_{2}^{\prime} ; \mathcal{B}, b_{1}^{\prime}, b_{2}^{\prime}\right)$ and the vertical games $\mathbf{G}_{m+2}^{3}\left(\mathcal{A}, a_{i}^{\prime}, a_{i} ; \mathcal{B}, b_{i}^{\prime}, b_{i}^{\prime}\right)$ for $i=1,2$.

Proof. For this proof since $\mathcal{A}$ and $\mathcal{B}$ are fixed, we will describe a game only by listing the chosen points, e.g., $\left(a_{1}, a_{2} ; b_{1}, b_{2}\right)$. We simulate two moves of the game, $\mathbf{G}_{m+4}^{3}\left(a_{1}, a_{2} ; b_{1}, b_{2}\right)$, in which we choose Player I's moves and then Player II answers according to her winning strategy. Let $u+v$ denote the least common ancestor of $u$ and $v$. First, we have Player I place pebble $x_{3}$ on $a_{1}^{\prime}$, the unique child of $a_{1}+a_{2}$ that is an ancestor of $a_{1}$. (Note that if $a_{1}^{\prime}=a_{1}$ then this move can be skipped and similarly for the second move if $a_{2}^{\prime}=a_{2}$.) Player II answers by placing $x_{3}$ on some point $b_{1}^{\prime}$. Second, Player I should move pebble $x_{1}$ from $a_{1}$ to $a_{2}^{\prime}$, the unique child of $a_{1}+a_{2}$ that is an ancestor of $a_{2}$. Player II moves $x_{1}$ to some point $b_{2}^{\prime}$.

Since Player II has moved according to her winning strategy, we have that she still has a winning strategy for the three games in the statement of the lemma. Furthermore, since $a_{1}^{\prime}$ and $a_{2}^{\prime}$ are siblings and we have two remaining moves, $b_{1}^{\prime}$ and $b_{2}^{\prime}$ must be siblings as well.

Using Lemma 6.3 we initially partition the universe according to four subgames:

- $\left(a_{r}, a_{p} ; b_{r}, b_{p}\right)$ with domain everything not below $a_{p}$ or $b_{p}$. Here $a_{p}=a_{1}+a_{2}$, i.e., the parent of $a_{1}^{\prime}, b_{p}=b_{1}+b_{2}$, i.e., the parent of $b_{1}^{\prime}$ and $a_{r}$ and $b_{r}$ are the roots of $\mathcal{A}$ and $\mathcal{B}$, (the roots are not necessary but then the subgames are all on horizontal or vertical pairs), or

- $\left(a_{1}^{\prime}, a_{1} ; b_{1}^{\prime}, b_{1}\right)$ with domain everything below $a_{1}^{\prime}$ or $b_{1}^{\prime}$,

- $\left(a_{2}^{\prime}, a_{2} ; b_{2}^{\prime}, b_{2}\right)$, with domain everything below $a_{2}^{\prime}$ or $b_{2}^{\prime}$,

- $\left(a_{1}^{\prime}, a_{2}^{\prime} ; b_{1}^{\prime}, b_{2}^{\prime}\right)$, with the remaining domain.

We now have to explain, inductively, how all moves of Player I in the $k$-pebble game are answered by Player II and how, in the process, the universe is further partitioned. We inductively assume that Player II has a winning strategy for each of the 3-pebble, m-move sub-games. There are two cases:

Vertical: Player I places a new pebble on a point $a$ that is in the domain of a vertical game: $\left(a_{1}, a_{2} ; b_{1}, b_{2}\right)$. We thus know that $a_{1}$ is a proper ancestor of $a$. The interesting case is where neither of $a$ and $a_{2}$ is above the other so, without loss of generality, assume that $a<a_{2}$. We place $x_{3}$ on $a_{2}^{\prime}$, the child of $a+a_{2}$ that is above $a_{2}$. Let Player II move according to her winning strategy, placing $x_{3}$ on some point $b_{2}^{\prime}$. We split the original game into $\left(a_{1}, a_{2}^{\prime} ; b_{1}, b_{2}^{\prime}\right)$ and $\left(a_{2}^{\prime}, a_{2} ; b_{2}^{\prime}, b_{2}\right)$ so Player II has a winning strategy for these 3 -pebble, $m-1$ move sub-games. Next, in the $\left(a_{1}, a_{2}^{\prime} ; b_{1}, b_{2}^{\prime}\right)$ game we place $x_{3}$ on $a_{p}$, the parent of 
$a_{2}^{\prime}$ and we let Player II answer according to her winning strategy, placing $x_{3}$ on some point, $b_{p}$. We then split off the game $\left(a_{1}, a_{p} ; b_{1}, b_{p}\right)$.

Returning to the game $\left(a_{1}, a_{2}^{\prime} ; b_{1}, b_{2}^{\prime}\right)$, we have Player I place $x_{3}$ on $a^{\prime}$, the sibling of $a_{2}^{\prime}$ above $a$, and let Player II answer according to her winning strategy, placing $x_{3}$ on some point, $b^{\prime}$.

Finally, we let Player I move $x_{1}$ to $a$, and let Player II reply with $x_{1}$ on some point $b$.

The sub-games are thus: $\left(a_{1}, a_{p} ; b_{1}, b_{p}\right),\left(a^{\prime}, a_{2}^{\prime} ; b^{\prime}, b_{2}^{\prime}\right),\left(a^{\prime}, a ; b^{\prime}, b\right)$, and $\left(a_{2}^{\prime}, a_{2} ; b_{2}^{\prime}, b_{2}\right)$ and Player II has winning strategies for the $\mathbf{G}_{m-3}^{3}$ game on all of them.

Horizontal: In this case, we have the configuration, $\left(a_{1}, a_{2} ; b_{1}, b_{2}\right)$, consisting of a pair of siblings. The only interesting case occurs when Player I puts a new pebble on some vertex, $a$, s.t. $a_{1}<a<a_{2}$. In this case, we have Player I place pebble $x_{3}$ on $a^{\prime}$, the sibling of $a_{1}$ above $a$. Player II will place pebble $x_{3}$ on some vertex, $b^{\prime}$, which must be a sibling of $b_{1}$ and $b_{2}$.

Next, in the game below $a^{\prime}$ and $b^{\prime}$, we let Player I place pebble $x_{2}$ on $a$ and we let Player II answer according to her winning strategy in this game, placing $x_{2}$ on some vertex, $b$. The domain of the original configuration is thus split into domains for three sub-games: $\left(a_{1}, a^{\prime} ; b_{1}, b^{\prime}\right),\left(a^{\prime}, a_{2} ; b^{\prime}, b_{2}\right)$, and $\left(a^{\prime}, a ; b^{\prime}, b\right)$. On each of these, Player II has a winning strategy for the 3 -pebble, $m-2$ move game.

We now complete the proof that Player II wins $\mathbf{G}_{k}^{k}\left(a_{1}, a_{2} ; b_{1}, b_{2}\right)$. Whenever Player I places a new pebble on some point, say $a$, in the original game, Player II will answer as described above, i.e., in one of the little games we will have Player II wins $\mathbf{G}_{3 r}^{3}\left(a, a^{\prime} ; b, b^{\prime}\right)$ where there are $r$ moves remaining in the big game.

Player II then answers in the big game by placing the corresponding pebble on $b$. To see that the resulting moves are a win for Player II, we must just consider any two pebbled points, $a_{i}, a_{j} \in \mathcal{A}$, and $b_{i}, b_{j} \in \mathcal{B}$. If they came from the same sub-game, then they agree on relations $\preceq_{\text {desc }}, \prec_{\text {sib }}$ because Player II wins the sub-game. Otherwise, $a_{i}, b_{i}$ came from one sub-game, $G_{i}$, and $a_{j}, b_{j}$ came from another sub-game, $G_{j}$. By our choice of the domains and transitivity of $\preceq_{\mathrm{desc}}, \prec_{\mathrm{sib}}$, it thus follows that $a_{i}, a_{j}$ stand in the same relation with respect to $\preceq_{\text {desc }}, \prec_{\text {sib }}$ as $b_{i}, b_{j}$ do.

6.2. The two-variable fragment. In this section, we construct a temporal logic that captures the two-variable fragment of FO over nested words. Note that for finite unranked trees, a navigational logic capturing $\mathrm{FO}^{2}$ is known [21, 20]: it corresponds to a fragment of XPath. However, translating the basic predicates over trees into the vocabulary of nested words requires 3 variables, and thus we cannot apply existing results even in the finite case.

Our temporal logic will be based on several next and eventually operators. Since FO ${ }^{2}$ over a linear ordering cannot define the successor relation but temporal logics have next operators, we explicitly introduce successors into the vocabulary of FO. These successor relations in effect partition the linear edges into three disjoint types; interior edges, call edges, and return edges, and the nesting edges (except those from a position to its linear successor) into two disjoint types; call-return summaries, and call-interior-return summaries.

- $S^{i}(i, j)$ holds iff $j=i+1$ and either $\mu(i, j)$ or $i$ is not a call and $j$ is not a return.

- $S^{c}(i, j)$ holds iff $i$ is a call and $j=i+1$ is not a return;

- $S^{r}(i, j)$ holds iff $i$ is not a call and $j=i+1$ is a return.

- $S^{c r}(i, j)$ holds iff $\mu(i, j)$ and there is a path from $i$ to $j$ using only call and return edges.

- $S^{c i r}(i, j)$ holds iff $\mu(i, j)$ and neither $j=i+1$ nor $S^{c r}(i, j)$. 
Let $T$ denote the set $\{c, i, r, c r, c i r\}$ of all edge types. In addition to the built-in predicates $S^{t}$ for $t \in T$, we add the transitive closure of all unions of subsets of these relations. That is, for each non-empty set $\Gamma \subseteq T$ of edge types, let $S^{\Gamma}$ stand for the union $\cup_{t \in \Gamma} S^{t}$, and let $\leq^{\Gamma}$ be the reflexive-transitive closure of $S^{\Gamma}$. Now when we refer to $\mathrm{FO}^{2}$ over nested words, we mean $\mathrm{FO}^{2}$ in the vocabulary of the unary predicates plus all the $\leq^{\Gamma}$ 's, the five successor relations, and the built-in unary call and ret predicates.

We define a temporal logic unary-NWTL that has future and past versions of next operators parameterized by edge types, and eventually operators parameterized by a set of edge types. For example, $\diamond\{c\}$ means eventually along a path containing only call edges. Its formulas are given by:

$$
\begin{aligned}
\varphi:= & \top|a| \text { call }|\operatorname{ret}| \neg \varphi\left|\varphi \vee \varphi^{\prime}\right| \\
& \bigcirc^{t} \varphi\left|\ominus^{t} \varphi\right| \diamond^{\Gamma} \varphi \mid \diamond^{\Gamma} \varphi
\end{aligned}
$$

where $a$ ranges over $\Sigma, t$ ranges over $T$, and $\Gamma$ ranges over non-empty subsets of $T$. The semantics is defined in the obvious way. For example, $(\bar{w}, i) \models{ }^{\Gamma} \varphi$ iff for some position $j, i \leq^{\Gamma} j$ and $(\bar{w}, j) \models \varphi ;(\bar{w}, i) \models \bigcirc^{t} \varphi$ iff for some position $j, S^{t}(i, j)$ and $(\bar{w}, j) \models \varphi$; and $(\bar{w}, i) \models \operatorname{call}$ iff $\operatorname{call}(i)$ holds in $\bar{w}$.

For an $\mathrm{FO}^{2}$ formula $\varphi(x)$ with one free variable $x$, let $q \operatorname{dp}(\varphi)$ be its quantifier depth, and for a unary-NWTL formula $\varphi^{\prime}$, let $\operatorname{odp}\left(\varphi^{\prime}\right)$ be its operator depth.

\section{Theorem 6.4.}

(1) unary-NWTL is expressively complete for $\mathrm{FO}^{2}$ over nested words.

(2) If formulas are viewed as DAGs (i.e identical subformulas are shared), then every $\mathrm{FO}^{2}$ formula $\varphi(x)$ can be converted to an equivalent unary-NWTL formula $\varphi^{\prime}$ of size $2^{\mathcal{O}(|\varphi|(\operatorname{qdp}(\varphi)+1))}$ and $\operatorname{odp}\left(\varphi^{\prime}\right) \leq 10 \mathrm{qdp}(\varphi)$. The translation is computable in time polynomial in the size of $\varphi^{\prime}$.

(3) Model checking of unary-NWTL can be carried out with the same worst case complexity as for $N W T L$.

Proof. The translation from unary-NWTL into $\mathrm{FO}^{2}$ is standard and can be done with negligible blow-up in the size of the formula, so we concentrate on the other direction. The proof generalizes the proof of an analogous result for unary temporal logic over words from [9].

Given an $\mathrm{FO}^{2}$ formula $\varphi(x)$ the translation procedure works a follows. When $\varphi(x)$ is atomic, i. e., of the form $a(x)$, it outputs $a$. When $\varphi(x)$ is of the form $\psi_{1} \vee \psi_{2}$ or $\neg \psi-$ we say that $\varphi(x)$ is composite - it recursively computes $\psi_{1}^{\prime}$ and $\psi_{2}^{\prime}$, or $\psi^{\prime}$ and outputs $\psi_{1}^{\prime} \vee \psi_{2}^{\prime}$ or $\neg \psi^{\prime}$. The two cases that remain are when $\varphi(x)$ is of the form $\exists x \varphi^{*}(x)$ or $\exists y \varphi^{*}(x, y)$. In both cases, we say that $\varphi(x)$ is existential. In the first case, $\varphi(x)$ is equivalent to $\exists y \varphi^{*}(y)$ and, viewing $x$ as a dummy free variable in $\varphi^{*}(y)$, this reduces to the second case.

In the second case, we can rewrite $\varphi^{*}(x, y)$ in the form

$$
\varphi^{*}(x, y)=\beta\left(\chi_{0}(x, y), . ., \chi_{r-1}(x, y), \xi_{0}(x), . ., \xi_{s-1}(x), \zeta_{0}(y), . ., \zeta_{t-1}(y)\right)
$$

where $\beta$ is a propositional formula, each formula $\chi_{i}$ is an atomic order formula, each formula $\xi_{i}$ is an atomic or existential $\mathrm{FO}^{2}$ formula with $\mathrm{qdp}\left(\xi_{i}\right)<\mathrm{qdp}(\varphi)$, and each formula $\zeta_{i}$ is an atomic or existential $\mathrm{FO}^{2}$ formula with $\operatorname{qdp}\left(\zeta_{i}\right)<\operatorname{qdp}(\varphi)$.

In order to be able to recurse on subformulas of $\varphi$ we have to separate the $\xi_{i}$ 's from the $\zeta_{i}$ 's. We first introduce a case distinction on which of the subformulas $\xi_{i}$ hold or not. We 
obtain the following equivalent formulation for $\varphi$ :

$$
\bigvee_{i \in\{T, \perp\}^{s}}\left(\bigwedge_{i<s}\left(\xi_{i} \leftrightarrow \gamma_{i}\right) \wedge \exists y \beta\left(\chi_{0}, \ldots, \chi_{r-1}, \gamma_{0}, \ldots, \gamma_{s-1}, \zeta_{0}, \ldots, \zeta_{t-1}\right)\right)
$$

We proceed by a case distinction on which order relation holds between $x$ and $y$, where $x \leq y$. We consider mutually exclusive cases, determined by the following formulas, which we call order types.

- $\Psi_{0}$ is $x=y$.

- For each $t \in T, \Psi_{t}$ is $S^{t}(x, y)$.

- For each $t \in T, \Phi_{t}$ is $\exists z\left(S^{t}(x, z) \wedge z<^{t} y\right)$.

- Let $o=t_{1}, t_{2}, \ldots t_{k}$ be a sequence over $T$ such that $2 \leq k \leq 5$, all $t_{i}$ 's are distinct, and a call never appears before return (that is, if $t_{i}=c$ then $t_{j} \neq r$ for $j>i$ ). Then $\Psi_{o}$ stands for

$$
\exists z_{1}, z_{1}^{\prime}, z_{2}, z_{2}^{\prime}, \ldots z_{k}\left(S^{t_{1}}\left(x, z_{1}\right) \wedge z_{1} \leq^{T_{1}} z_{1}^{\prime} \wedge S^{t_{2}}\left(z_{1}^{\prime}, z_{2}\right) \wedge z_{2} \leq^{T_{2}} z_{2}^{\prime} \wedge \cdots \wedge z_{k} \leq^{T_{k}} y\right)
$$

where for $1 \leq i \leq k$, the set $T_{i}$ equals the set $\left\{t_{1}, t_{2} \ldots t_{i}\right\}$, but with $r$ removed if both $c$ and $r$ belong to this set.

We claim that these order types are mutually exclusive and complete, and are expressible in unary-NWTL (and hence, in $\mathrm{FO}^{2}$ ). First, let us show that the order types form a disjoint partition, meaning for all pairs $(x, y)$ such that $x \leq y$, we have exactly one of these relationships holding true. To see this, suppose $x<y$. Then either $S^{t}(x, y)$ holds for some type $t$ (and the successor relations $S^{t}$ are disjoint, for distinct $t$ 's), or there is a path from $x$ to $y$ that uses at least two edges. The key observation is that a path from $x$ to $y$ is a summary path iff the path does not contain a call edge followed later by a return edge. Also, there is a unique summary path from $x$ to $y$. We can now classify the paths by the edge types that this unique summary path contains, and the order in which they first appear in the path. For example, $\Phi_{c}(x, y)$ holds when there is a path from $x$ to $y$ using 2 or more call edges; $\Phi_{c, c i r}(x, y)$ holds when there is a path from $x$ to $y$ which begins with a call edge, uses at least one call-interior-return summary edge, and uses only these two types of edges; $\Phi_{r, i, c}(x, y)$ holds when there is a path from $x$ to $y$ that can be split into three consecutive parts: a part containing only return edges, a part containing at least one internal and only internal and return edges, and a part containing at least one call and only call and internal edges. Note that some of these order types are empty: for example, two summary edges can never follow one another, and hence $\Phi_{c r}(x, y)$ can never hold. Emptiness of some of the order types is not relevant to the proof.

When we assume that one of these order types is true, each atomic order formula evaluates to either $T$ or $\perp$, in particular, each of the $\chi_{i}$ 's evaluates to either $T$ or $\perp$; we will denote this truth value by $\chi_{i}^{\tau}$. For example, when $\Psi_{c r}(x, y)$ holds then $(1) S^{t}(x, y)$ is true for $t=c r$ and false for $t \neq c r$, and $(2) \leq^{\Gamma}$ is true if $\Gamma$ contains $c r$ or if $\Gamma$ contains both $c$ and $r$, and false otherwise.

We can finally rewrite $\varphi$ as follows, where $\Upsilon$ stands for the set of all order types:

$$
\bigvee_{\bar{\gamma} \in\{T, \perp\}^{s}}\left(\bigwedge_{i<s}\left(\xi_{i} \leftrightarrow \gamma_{i}\right) \wedge \bigvee_{\tau \in \Upsilon} \exists y\left(\tau \wedge \beta\left(\chi_{0}^{\tau}, \ldots, \chi_{r-1}^{\tau}, \bar{\gamma}, \bar{\zeta}\right)\right)\right)
$$

If $\tau$ is an order type, $\psi(x)$ an $\mathrm{FO}^{2}$ formula, and $\psi^{\prime}$ an equivalent unary-NWTL formula, there is a way to obtain a unary-NWTL formula $\tau\langle\psi\rangle$ equivalent to $\exists y(\tau \wedge \psi(y))$, as follows. Assume that $x \leq y$. 
- For the order type $\Psi_{0}, \tau\left\langle\psi^{\prime}\right\rangle$ is $\psi^{\prime}$ itself.

- For each $t \in T$, for the order type $\Psi_{t}, \tau\left\langle\psi^{\prime}\right\rangle$ is $\bigcirc^{t} \psi^{\prime}$.

- For each $t \in T$, for the order type $\Phi_{t}, \tau\left\langle\psi^{\prime}\right\rangle$ is $\bigcirc^{t} \bigcirc^{t} \diamond^{\{t\}} \psi^{\prime}$.

- For order type $\Psi_{o}$, where $o=t_{1}, t_{2}, \ldots t_{k}$ is a sequence over $T, \tau\left\langle\psi^{\prime}\right\rangle$ is $\bigcirc^{t_{1}} \diamond^{T_{1}} \bigcirc^{t_{2}} \ldots$ $\diamond T_{k} \psi^{\prime}$, where for $1 \leq i \leq k \leq 5$, the set $T_{i}$ equals the set $\left\{t_{1}, t_{2} \ldots t_{i}\right\}$, but with $r$ removed if both $c$ and $r$ belong to this set.

The case corresponding to past operators is analogous. Our procedure will therefore recursively compute $\xi_{i}^{\prime}$ for $i<s$ and $\zeta_{i}^{\prime}(x)$ for $i<t$ and output

$$
\bigvee_{\bar{\gamma} \in\{T, \perp\}}\left(\bigwedge_{i<s}\left(\xi_{i}^{\prime} \leftrightarrow \gamma_{i}\right) \wedge \bigvee_{\tau \in \Upsilon} \tau\left\langle\beta\left(\chi_{0}^{\tau}, . ., \chi_{r-1}^{\tau}, \bar{\gamma}, \zeta_{0}^{\prime}(x), \ldots, \zeta_{t-1}^{\prime}(x)\right)\right\rangle\right)
$$

Now we verify that $\left|\varphi^{\prime}\right|$ and $\operatorname{odp}\left(\varphi^{\prime}\right)$ are bounded as stated in the theorem. Note that the size $\left|\varphi^{\prime}\right|$ is measured by viewing the unary-NWTL formula as a DAG, i.e., sharing identical subformulas. That $\operatorname{odp}\left(\varphi^{\prime}\right) \leq 10 \operatorname{qdp}(\varphi)$ is easily seen from the operator depth in the translation table above. The proof that $\left|\varphi^{\prime}\right| \leq 2^{c|\varphi|(\operatorname{qdp}(\varphi)+1)}$ for some constant $c$ is inductive on the quantifier depth of $\varphi$. The base case is trivial, and the only interesting case in the inductive step is when $\varphi$ is of the form $\exists y \varphi^{*}(x, y)$ as above. In this case, we have to estimate the length of (6.1). There are $2^{s} \leq 2^{|\varphi|}$ possibilities for $\bar{\gamma}$ in (6.1), and each disjunct in (6.1) has length at most $d|\varphi| \max _{i<s, j<t}\left(\left|\xi_{i}^{\prime}\right|,\left|\zeta_{j}^{\prime}\right|\right)$ for some constant $d$. By induction hypothesis, the latter is bounded by $d|\varphi| 2^{c|\varphi| q d p(\varphi)}$, which implies the claim, provided $c$ is chosen large enough.

It is straightforward to verify that our translation to $\varphi^{\prime}$ can be computed in time polynomial in $\left|\varphi^{\prime}\right|$.

Model checking of unary-NWTL can be achieved with the same complexity as for NWTL using a variant of the tableaux construction in Section 5 .

\section{Conclusion}

We have provided several new temporal logics over nested words and shown that they are first-order expressively complete. We have furthermore shown that first-order logic over nested words has the three-variable property, and we have also provided a temporal logic over nested words that is complete for two-variable first-order logic. We have shown, via an automata-theoretic approach based on nested word automata, that satisfiability for the logic $\mathrm{NWTL}^{+}$is EXPTIME-complete, and that model checking runs in time polynomial in the size of the RSM model and exponential in the size of the formula. When the within modality is added to NWTL, the complexity of model checking becomes doubly exponential. We note that it remains open whether the original temporal logic CaRet, proposed for nested words in [2, is first-order complete, but we conjecture that it is not.

\section{ACKNOWLEDGMENTS}

The authors were supported by: Alur - NSF CPA award 0541149; Arenas - FONDECYT grants 1050701, 7060172 and 1070732; Arenas and Barceló - grant P04-067-F from the Millennium Nucleus Centre for Web Research; Immerman - NSF grants CCF-0541018 and CCF-0830174; Libkin - EC grant MEXC-CT-2005-024502 and EPSRC grant E005039. 


\section{REFERENCES}

[1] R. Alur, M. Benedikt, K. Etessami, P. Godefroid, T. Reps, M. Yannakakis. Analysis of recursive state machines. ACM TOPLAS 27(4): 786-818 (2005).

[2] R. Alur, K. Etessami and P. Madhusudan. A temporal logic of nested calls and returns. In TACAS'04, pages $467-481$.

[3] R. Alur and P. Madhusudan. Visibly pushdown languages. In STOC'04, pages 202-211.

[4] R. Alur and P. Madhusudan. Adding nesting structure to words. In DLT'06, pages 1-13.

[5] T. Ball and S. Rajamani. Bebop: A symbolic model checker for boolean programs. In SPIN'00, pages $113-130$.

[6] V. Bárány, C. Lóding, O. Serre. Regularity problems for visibly pushdown languages. STACS 2006, pages $420-431$.

[7] Document Object Model DOM. http://www.w3.org/DOM.

[8] J. Esparza and S. Schwoon. A BDD-based model checker for recursive programs. In $C A V^{\prime} 01$, pages $324-336$.

[9] K. Etessami, M. Vardi, and T. Wilke. First-order logic with two variables and unary temporal logic. Information and Computation 179(2): 279-295, 2002.

[10] M. Frick, M. Grohe. The complexity of first-order and monadic second-order logic revisited. LICS 2002, 215-224.

[11] G. Gottlob, C. Koch. Monadic datalog and the expressive power of languages for web information extraction. Journal of the ACM 51 (2004), 74-113.

[12] N. Immerman. Descriptive Complexity. Springer, 1999.

[13] N. Immerman and D. Kozen. Definability with bounded number of bound variables. Information and Computation, 83 (1989), 121-139.

[14] H. Kamp. Tense Logic and the Theory of Linear Order. PhD thesis, UCLA, 1968.

[15] N. Klarlund, T. Schwentick and D. Suciu. XML: model, schemas, types, logics, and queries. In Logics for Emerging Applications of Databases, Springer, 2003, pages 1-41.

[16] L. Libkin. Logics for unranked trees: an overview. In ICALP 2005, pages 35-50.

[17] C. Löding, P. Madhusudan, O. Serre. Visibly pushdown games. In FSTTCS 2004, pages 408-420.

[18] P. Madhusudan, personal communication.

[19] M. Marx. Conditional XPath, the first order complete XPath dialect. In PODS'04, pages 13-22.

[20] M. Marx. Conditional XPath. TODS 30(4): 929-959, 2005.

[21] M. Marx and M. de Rijke. Semantic characterizations of navigational XPath. In TDM'04, pages 67-73.

[22] F. Neven, T. Schwentick. Expressive and efficient pattern languages for tree-structured data. PODS'O0, pages 145-156.

[23] F. Laroussinie, N. Markey, and P. Schnoebelen. Temporal logic with forgettable past. In LICS'02, pages 383-392.

[24] F. Neven and T. Schwentick. Query automata over finite trees. Theor. Comput. Sci. 275(1-2): 633-674, 2002.

[25] SAX: A Simple API for XML. http://www. saxproject.org.

[26] B.-H. Schlingloff. Expressive completeness of temporal logic of trees. J. Appl. Non-Classical Log. 2: 157-180, 1992.

[27] L. Segoufin. Typing and querying XML documents: some complexity bounds. In PODS'03, pages 167178.

[28] L. Segoufin, V. Vianu. Validating streaming XML documents. In PODS'02, pages 53-64.

[29] V. Vianu. A web Odyssey: from Codd to XML. In ACM PODS'01, pages 1-15. 


\section{Appendix A. Proof of Lemma 4.3}

For translating each NWTL ${ }^{s s}$ formula $\varphi$ into an equivalent NWTL formula $\beta_{\varphi}$, we need to consider only the case of until/since operators. The formula $\psi \mathbf{U}_{s s}^{\sigma} \theta$ is translated into

$$
\begin{aligned}
\beta_{\theta} \vee\left(\beta _ { \psi } \wedge \left(\left(\left(\beta_{\psi} \vee \text { ret }\right) \wedge\left(\neg \text { mcall } \rightarrow \bigcirc \beta_{\psi}\right) \wedge\left(\text { mcall } \rightarrow\left(\bigcirc_{\mu} \bigcirc \beta_{\psi} \vee \bigcirc_{\mu} \bigcirc \beta_{\theta}\right)\right)\right) \mathbf{U}^{\sigma}\right.\right. \\
\quad\left(\left(\beta_{\psi} \vee \text { ret }\right) \wedge\left(\neg \text { mcall } \rightarrow \bigcirc \beta_{\theta}\right) \wedge\right. \\
\qquad((\neg \text { ret } \wedge \gamma)))))),
\end{aligned}
$$

where $\gamma$ is a formula defined as follows:

$$
\begin{aligned}
&\left(\left(\beta_{\psi} \vee \text { ret }\right) \wedge\left(\neg \text { mcall } \rightarrow \bigcirc \beta_{\psi}\right) \wedge(\mathrm{mcall}\right.\left.\left.\rightarrow\left(\bigcirc \beta_{\psi} \vee \bigcirc_{\mu} \bigcirc \beta_{\psi}\right)\right) \wedge(\bigcirc \text { ret } \rightarrow \text { call })\right) \mathbf{U}^{\sigma} \\
&\left(\left(\beta_{\psi} \vee \text { ret }\right) \wedge\left(\neg \mathrm{mcall} \rightarrow \bigcirc \beta_{\theta}\right) \wedge\left(\mathrm{mcall} \rightarrow\left(\bigcirc \beta_{\theta} \vee \bigcirc_{\mu} \bigcirc \beta_{\theta}\right)\right)\right)
\end{aligned}
$$

The proof that the translation is correct is by induction on the structure of NWTL ${ }^{s s}$ formulas. Again we need to consider only the case of until/since operators. Assume that $\psi$, $\theta$ are equivalent to $\beta_{\psi}$ and $\beta_{\theta}$, respectively. We need to prove that $\psi \mathbf{U}_{s s}^{\sigma} \theta$ is equivalent to (A.1).

$(\Leftarrow)$ We first show that if $(\bar{w}, i)$ satisfies (A.1), then $(\bar{w}, i) \models \psi \mathbf{U}_{s s}^{\sigma} \theta$. Given that $(\bar{w}, i)$ satisfies (A.1), either $(\bar{w}, i) \models \beta_{\theta}$ or $(\bar{w}, i)$ satisfies the second disjunct of (A.1). Since $\beta_{\theta}$ and $\theta$ are assumed to be equivalent, in the former case $(\bar{w}, i) \models \psi \mathbf{U}_{s s}^{\sigma} \theta$. Thus, assume that the latter case holds. Then $(\bar{w}, i) \models \psi$, since $\psi$ and $\beta_{\psi}$ are equivalent, and there exists a summary path $i=i_{0}<i_{1}<\cdots<i_{p}$ such that:

$$
\begin{aligned}
& \left(\bar{w}, i_{k}\right) \models\left(\beta_{\psi} \vee \text { ret }\right) \wedge\left(\neg \text { mcall } \rightarrow \bigcirc \beta_{\psi}\right) \wedge\left(\text { mcall } \rightarrow\left(\bigcirc_{\mu} \bigcirc \beta_{\psi} \vee \bigcirc_{\mu} \bigcirc \beta_{\theta}\right)\right), \quad 0 \leq k<p, \\
& \left(\bar{w}, i_{p}\right) \models\left(\beta_{\psi} \vee \text { ret }\right) \wedge\left(\neg \text { mcall } \rightarrow \bigcirc \beta_{\theta}\right) \wedge\left(\text { mcall } \rightarrow\left(\bigcirc \beta_{\theta} \vee \bigcirc_{\mu} \bigcirc \beta_{\theta} \vee \bigcirc(\neg \text { ret } \wedge \gamma)\right)\right) .
\end{aligned}
$$

We consider three cases.

(I) Assume that there exists a position $i_{k}(k \in[0, p-1])$ such that $i_{k}$ is a matched call position and $\left(\bar{w}, i_{k}\right) \models \bigcirc_{\mu} \bigcirc \beta_{\theta}$, and let $i_{q}(q \in[0, p-1])$ be the first such position. Then only one semi-strict path with endpoints $i=i_{0}$ and $r\left(i_{q}\right)+1$ can be obtained from the sequence $i_{0}<i_{1}<\cdots<i_{q}<r\left(i_{q}\right)+1$ by removing all positions $i_{k}$ (with $k \in[1, q]$ ) such that $i_{k-1}$ is a matched call position and $r\left(i_{k-1}\right)=i_{k}$; let $i_{0}=j_{0}<j_{1}<\cdots<j_{\ell}=$ $r\left(i_{q}\right)+1$ be that semi-strict path. Next we show that:

$$
\begin{aligned}
\left(\bar{w}, j_{k}\right) & \models \psi \quad 0 \leq k<\ell, \\
\left(\bar{w}, j_{\ell}\right) & \models \theta,
\end{aligned}
$$

from which we conclude that $(\bar{w}, i) \models \psi \mathbf{U}_{s s}^{\sigma} \theta$.

Given that $j_{\ell}=r\left(i_{q}\right)+1,\left(\bar{w}, i_{q}\right) \models \bigcirc_{\mu} \bigcirc \beta_{\theta}$ and we assume that $\theta$ and $\beta_{\theta}$ are equivalent, we have that $\left(\bar{w}, j_{\ell}\right) \models \theta$. Next we show that $\left(\bar{w}, j_{k}\right) \models \psi$ for every $k \in$ $[0, \ell-1]$. If $k=0$, then the property holds since $(\bar{w}, i) \models \psi$ and we assume that $\psi$ and $\beta_{\psi}$ are equivalent. Assume that $k \in[1, \ell-1]$. If $j_{k}$ is not a return position, then $\left(\bar{w}, j_{k}\right) \models \psi$ since $\left(\bar{w}, j_{k}\right) \models \beta_{\psi} \vee$ ret (recall that $j_{k}$ is a position in the summary path $i_{0}<i_{1}<\cdots<i_{q}$ since $k<\ell$ ). If $j_{k}$ is a return position, then we have to consider two cases. If $j_{k-1}=j_{k}-1$, then we have that $j_{k}-1$ is not a call position since $j_{k}$ is 
a return position, $j_{0}<j_{1}<\cdots<j_{\ell}$ is a semi-strict path and $k-1 \in[0, \ell-2]$. Given that $j_{k-1}$ is a position in the summary path $i_{0}<i_{1}<\cdots<i_{q-1}$, we conclude that $\left(\bar{w}, j_{k}-1\right) \models \neg$ mcall $\rightarrow \bigcirc \beta_{\psi}$. Thus, from the fact that $j_{k-1}$ is not a call position, we conclude that $\left(\bar{w}, j_{k}\right) \models \beta_{\psi}$. Hence, $\left(\bar{w}, j_{k}\right) \models \psi$. Otherwise, $j_{k-1} \neq j_{k}-1$, and we conclude that $j_{k-1}$ is a matched call position and $j_{k}=r\left(j_{k-1}\right)+1$. Thus, since $i_{q}$ is the smallest one satisfying $\bigcirc_{\mu} \bigcirc \beta_{\theta}$ and $j_{k-1}<i_{q}$, and we know from (A.2) that $\left(\bar{w}, j_{k-1}\right) \models$ $\operatorname{mcall} \rightarrow\left(\bigcirc_{\mu} \bigcirc \beta_{\psi} \vee \bigcirc_{\mu} \bigcirc \beta_{\theta}\right)$, we see that $\left(\bar{w}, j_{k-1}\right) \models \operatorname{mcall} \rightarrow \bigcirc_{\mu} \bigcirc \beta_{\psi}$ and, since $j_{k-1}$ is a matched call, we conclude that $\left(\bar{w}, j_{k}\right) \models \beta_{\psi}$ and, therefore, $\left(\bar{w}, j_{k}\right) \models \psi$.

(II) Assume that condition (I) does not hold, and also assume that either $i_{p}$ is not a matched call position or $i_{p}$ is a matched call position and $\left(\bar{w}, i_{p}\right) \models \bigcirc \beta_{\theta} \vee \bigcirc_{\mu} \bigcirc \beta_{\theta}$. Then given that $\left(\bar{w}, i_{p}\right) \models \neg$ mcall $\rightarrow \bigcirc \beta_{\theta}$, we have that there exists a position $i_{p+1}>i_{p}$ such that $\left(\bar{w}, i_{p+1}\right) \models \beta_{\theta}$ and $i_{p+1}$ is either $i_{p}+1$ or $r\left(i_{p}\right)+1$. Only one semi-strict path with endpoints $i=i_{0}$ and $i_{p+1}$ can be obtained from the sequence $i_{0}<i_{1}<\cdots<i_{p}<i_{p+1}$ by removing all positions $i_{k}$ (with $k \in[1, p]$ ) such that $i_{k-1}$ is a matched call position and $r\left(i_{k-1}\right)=i_{k}$; let $i_{0}=j_{0}<j_{1}<\cdots<j_{\ell}=i_{p+1}$ be that semi-strict path. Next we show that:

$$
\begin{aligned}
\left(\bar{w}, j_{k}\right) & \models \psi \quad 0 \leq k<\ell, \\
\left(\bar{w}, j_{\ell}\right) & \models \theta,
\end{aligned}
$$

from which we conclude that $(\bar{w}, i) \models \psi \mathbf{U}_{s s}^{\sigma} \theta$.

Given that $\left(\bar{w}, i_{p+1}\right) \models \beta_{\theta}$ and the hypothesis that $\theta$ and $\beta_{\theta}$ are equivalent, we have that $\left(\bar{w}, j_{\ell}\right) \models \theta$. Next we show that $\left(\bar{w}, j_{k}\right) \models \psi$ for every $k \in[0, \ell-1]$. If $k=0$, then the property holds since $(\bar{w}, i) \models \psi$. Assume that $k \in[1, \ell-1]$. If $j_{k}$ is not a return position, then $\left(\bar{w}, j_{k}\right) \models \psi$ since $\left(\bar{w}, j_{k}\right) \models \beta_{\psi} \vee$ ret (recall that $j_{k}$ is a position in the summary path $i_{0}<i_{1}<\cdots<i_{p}$ ). If $j_{k}$ is a return position, then we have to consider two cases. If $j_{k-1}=j_{k}-1$, then we have that $j_{k}-1$ is not a call position since $j_{0}<j_{1}<\cdots<j_{\ell}$ is a semi-strict path and $k-1 \in[0, \ell-2]$. Thus, given that $j_{k-1}$ is a position in the summary path $i_{0}<i_{1}<\cdots<i_{p-1}$, we have that $\left(\bar{w}, j_{k}-1\right) \models \neg$ mcall $\rightarrow \bigcirc \beta_{\psi}$, from which we conclude that $\left(\bar{w}, j_{k}\right) \models \beta_{\psi}$. Hence, $\left(\bar{w}, j_{k}\right) \models \psi$. Otherwise, $j_{k-1} \neq j_{k}-1$, and we conclude that $j_{k-1}$ is a matched call position and $j_{k}=r\left(j_{k-1}\right)+1$. Thus, given that condition (I) does not hold, we have that $\left(\bar{w}, j_{k-1}\right) \models$ mcall $\rightarrow \bigcirc_{\mu} \bigcirc \beta_{\psi}$ (since $j_{k-1}$ is a position in the summary path $i_{0}<i_{1}<\cdots<i_{p-1}$ and $\left.\left(\bar{w}, j_{k-1}\right) \not \models \bigcirc_{\mu} \bigcirc \beta_{\theta}\right)$. Thus, given that $j_{k-1}$ is a matched call, we conclude from (A.2) that $\left(\bar{w}, j_{k}\right) \models \beta_{\psi}$ and, therefore, $\left(\bar{w}, j_{k}\right) \models \psi$.

(III) We now look at the remaining cases, that is, condition (I) does not hold, $i_{p}$ is a matched call, and $\left(\bar{w}, i_{p}\right) \models \neg\left(\bigcirc \beta_{\theta} \vee \bigcirc_{\mu} \bigcirc \beta_{\theta}\right)$. By (A.3), this implies $\left(\bar{w}, i_{p}\right) \models \bigcirc(\neg$ ret $\wedge \gamma)$. From $\left(\bar{w}, i_{p}\right) \models \bigcirc \gamma$, we see that there exists a summary path $i_{p}+1=i_{p+1}<i_{p+2} \ldots<i_{q}$ such that:

$$
\begin{aligned}
\left(\bar{w}, i_{k}\right) \models\left(\beta_{\psi} \vee \operatorname{ret}\right) \wedge & \left(\neg \text { mcall } \rightarrow \bigcirc \beta_{\psi}\right) \wedge \\
& \quad\left(\operatorname{mcall} \rightarrow\left(\bigcirc \beta_{\psi} \vee \bigcirc_{\mu} \bigcirc \beta_{\psi}\right)\right) \wedge(\bigcirc \text { ret } \rightarrow \text { call }) \quad p+1 \leq k<q, \\
\left(\bar{w}, i_{q}\right) \models\left(\beta_{\psi} \vee \operatorname{ret}\right) \wedge\left(\neg \text { mcall } \rightarrow \bigcirc \beta_{\theta}\right) \wedge\left(\operatorname{mcall} \rightarrow\left(\bigcirc \beta_{\theta} \vee \bigcirc_{\mu} \bigcirc \beta_{\theta}\right)\right) . &
\end{aligned}
$$

We first show that $i_{q}<r\left(i_{p}\right)$. Assume to the contrary that $i_{q} \geq r\left(i_{p}\right)$. Since the first position on the path is inside the call $i_{p}$, there exists $k \in[p+1, q]$ such that $i_{k}=r\left(i_{p}\right)$. Given that $i_{p+1}$ is not a return position ( since $\left(\bar{w}, i_{p}\right) \models \neg$ Oret), we have that $q>p+1$ and, therefore, $i_{k}-1$ is also a position in the summary path $i_{p+1}<i_{p+2} \ldots<i_{q}$. But given that $i_{k}=r\left(i_{p}\right)$ is the matching return of $i_{p}$ and $i_{p}+1 \leq i_{k}-1$, we have that 
$i_{k}-1$ is not a call position. Thus, $\left(\bar{w}, i_{k}-1\right) \not \forall$ Oret $\rightarrow$ call, which contradicts the fact that $i_{p+1}<i_{p+2} \ldots<i_{q}$ witnesses formula $\gamma$. Therefore indeed $i_{q}<r\left(i_{p}\right)$.

Given that $\left(\bar{w}, i_{q}\right) \models\left(\neg \operatorname{mcall} \rightarrow \bigcirc \beta_{\theta}\right) \wedge\left(\operatorname{mcall} \rightarrow\left(\bigcirc \beta_{\theta} \vee \bigcirc_{\mu} \bigcirc \beta_{\theta}\right)\right)$, we conclude that there exists a position $i_{q+1}>i_{q}$ such that $\left(\bar{w}, i_{q+1}\right) \models \beta_{\theta}$ and $i_{q+1}$ is either $i_{q}+1$ or $r\left(i_{q}\right)+1$. Only one semi-strict path with endpoints $i=i_{0}$ and $i_{q+1}$ can be obtained from the sequence $i_{0}<i_{1}<\cdots<i_{q}<i_{q+1}$ by removing all positions $i_{k}$ (with $k \in[1, q]$ ) such that $i_{k-1}$ is a matched call position and $r\left(i_{k-1}\right)=i_{k}$; let $i_{0}=j_{0}<j_{1}<\cdots<j_{\ell}=i_{q+1}$ be that semi-strict path. Next we show that:

$$
\begin{aligned}
& \left(\bar{w}, j_{k}\right) \models \psi \quad 0 \leq k<\ell, \\
& \left(\bar{w}, j_{\ell}\right) \models \theta,
\end{aligned}
$$

from which we conclude that $(\bar{w}, i) \models \psi \mathbf{U}_{s s}^{\sigma} \theta$.

Given that $\left(\bar{w}, i_{q+1}\right) \models \beta_{\theta}$, we conclude that that $\left(\bar{w}, j_{\ell}\right) \models \theta$. Next we show that $\left(\bar{w}, j_{k}\right) \models \psi$ for every $k \in[0, \ell-1]$. If $k=0$, then the property holds since $(\bar{w}, i) \models \psi$ and we assume that $\psi$ and $\beta_{\psi}$ are equivalent. Assume that $k \in[1, \ell-1]$. If $j_{k}$ is not a return position, then $\left(\bar{w}, j_{k}\right) \models \psi$ since $\left(\bar{w}, j_{k}\right) \models \beta_{\psi} \vee$ ret (recall that $j_{k}$ is a position in the sequence $i_{0}<i_{1}<\cdots<i_{q}$ ). If $j_{k}$ is a return position, then we need to consider two cases. If $j_{k-1}=j_{k}-1$, then we have that $j_{k}-1$ is not a call position since $j_{0}<j_{1}<\cdots<j_{\ell}$ is a semi-strict path and $k-1 \in[0, \ell-2]$. Thus, given that $j_{k-1}$ is a position in the sequence $i_{0}<i_{1}<\cdots<i_{q-1}$ and $j_{k-1} \neq i_{p}$ (since $i_{p}$ is a call position), we have that $\left(\bar{w}, j_{k}-1\right) \models \neg$ mcall $\rightarrow \bigcirc \beta_{\psi}$, from which we conclude that $\left(\bar{w}, j_{k}\right) \models \beta_{\psi}$. Hence, $\left(\bar{w}, j_{k}\right) \models \psi$. If $j_{k-1} \neq j_{k}-1$, then we have that $j_{k-1}$ is a matched call position and $j_{k}=r\left(j_{k-1}\right)+1$. Moreover, in this case we also have that $j_{k}<i_{p}$. Indeed, to see this, assume to the contrary that $i_{p} \leq j_{k}$. Then given that $i_{q}<r\left(i_{p}\right)$, we know that $i_{q+1} \leq r\left(i_{p}\right)$. Thus, given that $i_{p}$ is a call position, $k<\ell$ and $j_{\ell}=i_{q+1}$, we conclude that $i_{p+1} \leq j_{k} \leq i_{q}$. Therefore, given that $j_{k}$ is a return position and $i_{p+1}<\cdots<i_{q}$ is a summary path, there exists $s \in[p+1, q]$ such that $i_{s}$ is a call position with matching return $j_{k}$. But since $j_{k-1}$ and $j_{k}$ are both positions in the summary path $i_{p+1}<\cdots<i_{q}$ and $j_{k}=r\left(j_{k-1}\right)+1$, we conclude that this path contains three positions $a, b$ and $c$ such that $a<b<c$ and $c$ is the matching return of call position $a$, which contradicts the definition of summary path. So we proved $j_{k}<i_{p}$. Now we have that $\left(\bar{w}, j_{k-1}\right) \models \operatorname{mcall} \rightarrow\left(\bigcirc_{\mu} \bigcirc \beta_{\psi} \vee \bigcirc_{\mu} \bigcirc \beta_{\theta}\right)$, from which we conclude that $\left(\bar{w}, j_{k}\right) \models \beta_{\psi}$ since condition (I) does not hold and $j_{k}=r\left(j_{k-1}\right)+1$. Hence, $\left(\bar{w}, j_{k}\right) \models \psi$.

$(\Rightarrow)$ We now show that if $(\bar{w}, i) \models \psi \mathbf{U}_{s s}^{\sigma} \theta$, then $(\bar{w}, i)$ satisfies (A.1). Given that $(\bar{w}, i) \models$ $\psi \mathbf{U}_{s s}^{\sigma} \theta$, there exists a semi-strict path $i=i_{0}<i_{1}<\cdots<i_{q}$ such that:

$$
\begin{aligned}
& \left(\bar{w}, i_{k}\right) \models \psi \quad 0 \leq k<q, \\
& \left(\bar{w}, i_{q}\right) \models \theta .
\end{aligned}
$$

Notice that if $q=0$, then $(\bar{w}, i) \models \theta$ and, therefore, $(\bar{w}, i)$ satisfies the first disjunct of (A.1) since $\theta$ and $\beta_{\theta}$ are assumed to be equivalent. Thus, we suppose that $q>0$, and we consider two cases.

(I) Assume that there exists $k \in[0, q-1]$ such that $i_{k}$ is a matched call position, $i_{k+1}=i_{k}+1$ and $i_{k+1}$ is not a return position, and let $i_{p}$ be the first such position. Then only one summary path with endpoints $i=i_{0}$ and $i_{p}$ can be obtained from the semi-strict path $i_{0}<i_{1}<\cdots<i_{p}$ by adding positions $r\left(i_{k}\right)$ for every $k \in[0, p-1]$ such that $i_{k}$ is a 
matched call position and $i_{k+1}=r\left(i_{k}\right)+1$; let $i_{0}=j_{0}<j_{1}<\cdots<j_{\ell}=i_{p}$ be that summary path. Next we show that:

$$
\begin{aligned}
& \left(\bar{w}, j_{k}\right) \models\left(\beta_{\psi} \vee \text { ret }\right) \wedge\left(\neg \text { mcall } \rightarrow \bigcirc \beta_{\psi}\right) \wedge\left(\text { mcall } \rightarrow \bigcirc_{\mu} \bigcirc \beta_{\psi}\right) \quad 0 \leq k<\ell, \\
& \left(\bar{w}, j_{\ell}\right) \models\left(\beta_{\psi} \vee \text { ret }\right) \wedge\left(\neg \text { mcall } \rightarrow \bigcirc \beta_{\theta}\right) \wedge\left(\text { mcall } \rightarrow\left(\bigcirc \beta_{\theta} \vee \bigcirc_{\mu} \bigcirc \beta_{\theta} \vee \bigcirc(\neg \text { ret } \wedge \gamma)\right)\right),
\end{aligned}
$$

from which we conclude that $(\bar{w}, i)$ satisfies $($ A.1).

We start by showing that the first condition above holds. Let $k \in[0, \ell-1]$. If $j_{k}$ is a return position, then we have that $\left(\bar{w}, j_{k}\right) \models\left(\beta_{\psi} \vee\right.$ ret $)$. Otherwise, by definition of $j_{0}<\ldots<j_{\ell}$, we have that $j_{k}$ is a position in the semi-strict path $i_{0}<i_{1}<\cdots<i_{p-1}$. Thus, from (A.4) we conclude that $\left(\bar{w}, j_{k}\right) \models \psi$ and, hence, $\left(\bar{w}, j_{k}\right) \models\left(\beta_{\psi} \vee\right.$ ret) since $\psi$ and $\beta_{\psi}$ are assumed to be equivalent. It only remains to show that $\left(\bar{w}, j_{k}\right) \models$ $\left(\neg\right.$ mcall $\left.\rightarrow \bigcirc \beta_{\psi}\right) \wedge\left(\right.$ mcall $\left.\rightarrow \bigcirc_{\mu} \bigcirc \beta_{\psi}\right)$. If $j_{k}$ is a matched call position, then by definition of $i_{p}$ we have that $j_{k}$ and $r\left(j_{k}\right)+1$ are both positions in the semi-strict path $i_{0}<i_{1}<\cdots<i_{p-1}$. Thus, from (A.4) we conclude that $\left(\bar{w}, r\left(j_{k}\right)+1\right) \models \psi$ and, therefore, $\left(\bar{w}, j_{k}\right) \models\left(\right.$ mcall $\left.\rightarrow \bigcirc_{\mu} \bigcirc \beta_{\psi}\right)$. If $j_{k}$ is not a matched call position, then we have that $j_{k}+1$ is a position in the semi-strict path $i_{0}<i_{1}<\cdots<i_{p}$. Thus, from (A.4) we conclude that $\left(\bar{w}, j_{k}+1\right) \models \psi$ and, therefore, $\left(\bar{w}, j_{k}\right) \models\left(\neg\right.$ mcall $\left.\rightarrow \bigcirc \beta_{\psi}\right)$.

We now show that the second condition above also holds. Given that $j_{\ell}=i_{p}$ is a matched call position, we have to prove that $\left(\bar{w}, j_{\ell}\right) \models \beta_{\psi} \wedge\left(\bigcirc \beta_{\theta} \vee \bigcirc_{\mu} \bigcirc \beta_{\theta} \vee \bigcirc(\neg\right.$ ret $\wedge$ $\gamma)$ ). Given that $\left(\bar{w}, i_{p}\right) \models \psi$ and we assume that $\psi$ and $\beta_{\psi}$ are equivalent, we have that $\left(\bar{w}, j_{\ell}\right) \models \beta_{\psi}$. If $q=p+1$, then given that $\left(\bar{w}, i_{q}\right) \models \theta$ and we assume that $\theta$ and $\beta_{\theta}$ are equivalent, we conclude that $\left(\bar{w}, j_{\ell}\right) \models \bigcirc \beta_{\theta}$. Thus, assume that $q>p+1$. Next we show that $\left(\bar{w}, j_{\ell}\right) \models \bigcirc(\neg$ ret $\wedge \gamma)$ in this case. Given that $i_{p+1}=i_{p}+1$ and $i_{p+1}$ is not a return position, we have $\left(\bar{w}, i_{p}+1\right) \models \neg$ ret, and it only remains to prove that $\left(\bar{w}, i_{p}+1\right) \models \gamma$. Given that $q>p+1$, only one summary path with endpoints $i_{p}+1$ and $i_{q-1}$ can be obtained from the sequence $i_{p}+1=i_{p+1}<i_{p+2}<\cdots<i_{q-1}$ by adding positions $r\left(i_{k}\right)$ for every $k \in[p+1, q-2]$ such that $i_{k}$ is a call position and $i_{k+1}=r\left(i_{k}\right)+1$; let $i_{p}+1=s_{0}<s_{1}<\cdots<s_{m}=i_{q-1}$ be that summary path. Next we show that:

$$
\begin{aligned}
&\left(\bar{w}, s_{k}\right) \models\left(\beta_{\psi} \vee \text { ret }\right) \wedge(\neg \operatorname{mcall}\left.\rightarrow \bigcirc \beta_{\psi}\right) \wedge \\
&\left(\operatorname{mcall} \rightarrow\left(\bigcirc \beta_{\psi} \vee \bigcirc_{\mu} \bigcirc \beta_{\psi}\right)\right) \wedge(\bigcirc \text { ret } \rightarrow \operatorname{call}) \quad 0 \leq k<m, \\
&\left(\bar{w}, s_{m}\right) \models\left(\beta_{\psi} \vee \text { ret }\right) \wedge\left(\neg \text { mcall } \rightarrow \bigcirc \beta_{\theta}\right) \wedge\left(\operatorname{mcall} \rightarrow\left(\bigcirc \beta_{\theta} \vee \bigcirc_{\mu} \bigcirc \beta_{\theta}\right)\right) .
\end{aligned}
$$

from which we conclude that $\left(\bar{w}, i_{p}+1\right) \models \gamma$.

We start by showing that the first condition above holds. Let $k \in[0, m-1]$. If $s_{k}$ is a return position, then we have that $\left(\bar{w}, s_{k}\right) \models\left(\beta_{\psi} \vee\right.$ ret $)$. Otherwise, by definition of $s_{0}<\ldots<s_{m}$, we have that $s_{k}$ is a position in the semi-strict path $i_{p+1}<i_{p+2}<\cdots<$ $i_{q-1}$. Thus, from (A.4) we conclude that $\left(\bar{w}, s_{k}\right) \models \psi$ and, hence, $\left(\bar{w}, s_{k}\right) \models\left(\beta_{\psi} \vee\right.$ ret $)$ since $\psi$ and $\beta_{\psi}$ are assumed to be equivalent. It only remains to show that:

$$
\left(\bar{w}, s_{k}\right) \models\left(\neg \mathrm{mcall} \rightarrow \bigcirc \beta_{\psi}\right) \wedge\left(\mathrm{mcall} \rightarrow\left(\bigcirc \beta_{\psi} \vee \bigcirc_{\mu} \bigcirc \beta_{\psi}\right)\right) \wedge(\bigcirc \mathrm{ret} \rightarrow \mathrm{call}) .
$$

If $s_{k}$ is a matched call position, then $s_{k}$ is a position in semi-strict path $i_{p+1}<i_{p+2}<$ $\cdots<i_{q-1}$ and either (a) $s_{k+1}=s_{k}+1$ and $s_{k}+1$ is a position in the semi-strict path $i_{p+1}<i_{p+2}<\cdots<i_{q-1}$, or (b) $s_{k+1}=r\left(s_{k}\right)$ and $r\left(s_{k}\right)+1$ is a position in the semistrict path $i_{p+1}<i_{p+2}<\cdots<i_{q-1}$. In the former case, from (A.4 we conclude that $\left(\bar{w}, s_{k}+1\right) \models \psi$ and, therefore, $\left(\bar{w}, s_{k}\right) \models$ mcall $\rightarrow \bigcirc \beta_{\psi}$. In the latter case, from (A.4) we conclude that $\left(\bar{w}, r\left(s_{k}\right)+1\right) \models \psi$ and, therefore, $\left(\bar{w}, s_{k}\right) \models$ mcall $\rightarrow \bigcirc_{\mu} \bigcirc \beta_{\psi}$. Thus, if $s_{k}$ is a matched call position, then $\left(\bar{w}, s_{k}\right) \models \operatorname{mcall} \rightarrow\left(\bigcirc \beta_{\psi} \vee \bigcirc_{\mu} \bigcirc \beta_{\psi}\right)$. Assume now that $s_{k}$ is not a matched call position. Given that $i_{0}<\cdots<i_{p}<i_{p+1}<\cdots<i_{q}$ 
is a semi-strict path, $i_{p}$ is a matched call position, $i_{p+1}=i_{p}+1$ and $i_{p}+1$ is not a return position, we have that $i_{q} \leq r\left(i_{p}\right)$. Thus, given that $s_{k}<i_{q}$, we have that $i_{p}<s_{k}<r\left(i_{p}\right)$, which implies that $s_{k}$ is either an internal position or a return position. Therefore, $s_{k}+1$ is a position in the semi-strict path $i_{p+1}<i_{p+2}<\cdots<i_{q-1}$ and, thus, from (A.4) we conclude that $\left(\bar{w}, s_{k}+1\right) \models \psi$. Hence, $\left(\bar{w}, s_{k}\right) \models \neg$ mcall $\rightarrow \bigcirc \beta_{\psi}$, and it only remains to prove that $\left(\bar{w}, s_{k}\right) \models$ Oret $\rightarrow$ call. On the contrary, assume that $\left(\bar{w}, s_{k}\right) \models$ Oret and $\left(\bar{w}, s_{k}\right) \not \models$ call. Given that $s_{k}$ is not a call position and $s_{k}<i_{q-1}$, we have that $s_{k}+1$ is a position in the semi-strict path $i_{p+1}<\cdots<i_{q-1}$. Thus, given that $\left(\bar{w}, s_{k}\right) \models$ Oret, we conclude that there exists a return position in the sequence $i_{p}+1<\cdots<i_{q-1}$. But this leads to a contradiction since from the fact that $i_{q} \leq r\left(i_{p}\right)$, we can conclude that none of the elements $i_{p+1}, \ldots, i_{q-1}$ is a return position.

To conclude this part of the proof, we need to show that the second condition above holds, that is, $\left(\bar{w}, s_{m}\right) \models\left(\beta_{\psi} \vee\right.$ ret $) \wedge\left(\neg\right.$ mcall $\left.\rightarrow \bigcirc \beta_{\theta}\right) \wedge\left(\operatorname{mcall} \rightarrow\left(\bigcirc \beta_{\theta} \vee \bigcirc_{\mu} \bigcirc \beta_{\theta}\right)\right)$. Given that $s_{m}=i_{q-1}$, we have that $\left(\bar{w}, s_{m}\right) \models \psi$ and, therefore, $\left(\bar{w}, s_{m}\right) \models \beta_{\psi} \vee$ ret. It remains to show that $\left(\bar{w}, s_{m}\right) \models\left(\neg \operatorname{mcall} \rightarrow \bigcirc \beta_{\theta}\right) \wedge\left(\operatorname{mcall} \rightarrow\left(\bigcirc \beta_{\theta} \vee \bigcirc_{\mu} \bigcirc \beta_{\theta}\right)\right)$. Given that $i_{q} \leq r\left(i_{p}\right)$, we know that $s_{m}=i_{q-1}$ is not a return position. If $s_{m}$ is an internal position, then $i_{q}=i_{q-1}+1$ and, thus, $\left(\bar{w}, s_{m}\right) \models \neg$ mcall $\rightarrow \bigcirc \beta_{\theta}$ since $\left(\bar{w}, i_{q}\right) \models \theta$. If $s_{m}$ is a call position, then $s_{m}$ has a matching return and either $i_{q}=i_{q-1}+1$ or $i_{q}=$ $r\left(i_{q-1}\right)+1$. In the former case, we have that $\left(\bar{w}, s_{m}\right) \models \operatorname{mcall} \rightarrow \bigcirc \beta_{\theta}$ since $\left(\bar{w}, i_{q}\right) \models \theta$. In the latter case, we have that $\left(\bar{w}, s_{m}\right) \models \operatorname{mcall} \rightarrow \bigcirc_{\mu} \bigcirc \beta_{\theta}$ since $\left(\bar{w}, i_{q}\right) \models \theta$. Hence, we conclude that $\left(\bar{w}, s_{m}\right) \models \operatorname{mcall} \rightarrow\left(\bigcirc \beta_{\theta} \vee \bigcirc_{\mu} \bigcirc \beta_{\theta}\right)$.

(II) Assume that condition (I) does not hold, that is, assume that there is no $k \in[0, q-$ 1] such that $i_{k}$ is a matched call position, $i_{k+1}=i_{k}+1$ and $i_{k+1}$ is not a return position. Then only one summary path with endpoints $i=i_{0}$ and $i_{q}$ can be obtained from the semi-strict path $i_{0}<i_{1}<\cdots<i_{q}$ by adding positions $r\left(i_{k}\right)$ for every $k \in[0, q-1]$ such that $i_{k}$ is a matched call position and $i_{k+1}=r\left(i_{k}\right)+1$; let $i_{0}=j_{0}<j_{1}<\cdots<j_{\ell}=i_{q}$ be that summary path. Next we show that: $\left(\bar{w}, j_{k}\right) \models\left(\beta_{\psi} \vee\right.$ ret $) \wedge\left(\neg\right.$ mcall $\left.\rightarrow \bigcirc \beta_{\psi}\right) \wedge\left(\right.$ mcall $\left.\rightarrow\left(\bigcirc_{\mu} \bigcirc \beta_{\psi} \vee \bigcirc_{\mu} \bigcirc \beta_{\theta}\right)\right) \quad 0 \leq k<\ell-1$, $\left(\bar{w}, j_{\ell-1}\right) \models\left(\beta_{\psi} \vee\right.$ ret $) \wedge\left(\neg\right.$ mcall $\left.\rightarrow \bigcirc \beta_{\theta}\right) \wedge\left(\right.$ mcall $\rightarrow\left(\bigcirc \beta_{\theta} \vee \bigcirc_{\mu} \bigcirc \beta_{\theta} \vee \bigcirc(\neg\right.$ ret $\left.\left.\wedge \gamma)\right)\right)$, from which we conclude that $(\bar{w}, i)$ satisfies (A.1).

We start by showing that the first condition above holds. Let $k \in[0, \ell-2]$. If $j_{k}$ is a return position, then we have that $\left(\bar{w}, j_{k}\right) \models\left(\beta_{\psi} \vee\right.$ ret $)$. Otherwise, by definition of $j_{0}<\ldots<j_{\ell}$, we have that $j_{k}$ is a position in the semi-strict path $i_{0}<i_{1}<\cdots<i_{q-1}$. Thus, from (A.4 we conclude that $\left(\bar{w}, j_{k}\right) \models \psi$ and, hence, $\left(\bar{w}, j_{k}\right) \models\left(\beta_{\psi} \vee\right.$ ret) since $\psi$ and $\beta_{\psi}$ are assumed to be equivalent. It only remains to show that $\left(\bar{w}, j_{k}\right) \models$ $\left(\neg\right.$ mcall $\left.\rightarrow \bigcirc \beta_{\psi}\right) \wedge\left(\operatorname{mcall} \rightarrow\left(\bigcirc_{\mu} \bigcirc \beta_{\psi} \vee \bigcirc_{\mu} \bigcirc \beta_{\theta}\right)\right)$. If $j_{k}$ is a matched call position and $k<\ell-2$, then given that $i_{0}<i_{1}<\cdots<i_{q}$ is a semi-strict path and condition (I) does not hold, we have that $j_{k}$ and $r\left(j_{k}\right)+1$ are both positions in the semi-strict path $i_{0}<i_{1}<\cdots<i_{q-1}$. Thus, from (A.4) we conclude that $\left(\bar{w}, r\left(j_{k}\right)+1\right) \models \psi$ and, therefore, $\left(\bar{w}, j_{k}\right) \models$ mcall $\rightarrow \bigcirc_{\mu} \bigcirc \beta_{\psi}$. If $j_{k}$ is a matched call position and $k=\ell-2$, then given that $i_{0}<i_{1}<\cdots<i_{q}$ is a semi-strict path and condition (I) does not hold, we have that $j_{\ell-1}=r\left(j_{k}\right)$ and $i_{q}=j_{\ell}=r\left(j_{k}\right)+1$. Thus, given that $\left(\bar{w}, i_{q}\right) \models \theta$, we conclude that $\left(\bar{w}, j_{k}\right) \models \operatorname{mcall} \rightarrow \bigcirc_{\mu} \bigcirc \beta_{\theta}$. Finally, if $j_{k}$ is not a matched call position, then we have that $j_{k}+1$ is a position in the semi-strict path $i_{0}<i_{1}<\cdots<i_{q-1}$ (since $k<\ell-1)$. Thus, from (A.4) we conclude that $\left(\bar{w}, j_{k}+1\right) \models \psi$ and, therefore, $\left(\bar{w}, j_{k}\right) \models \neg$ mcall $\rightarrow \bigcirc \beta_{\psi}$. 
To conclude the proof of the lemma, we show that the second condition above also holds, that is, $\left(\bar{w}, j_{\ell-1}\right) \models\left(\beta_{\psi} \vee\right.$ ret $) \wedge\left(\neg\right.$ mcall $\left.\rightarrow \bigcirc \beta_{\theta}\right) \wedge\left(\right.$ mcall $\rightarrow\left(\bigcirc \beta_{\theta} \vee \bigcirc \mu \bigcirc \beta_{\theta} \vee\right.$ $\bigcirc(\neg$ ret $\wedge \gamma)))$. If $j_{\ell-1}$ is a return position, we immediately conclude that $\left(\bar{w}, j_{\ell-1}\right) \models$ $\left(\beta_{\psi} \vee\right.$ ret). Thus, assume that $j_{\ell-1}$ is not a return position. But in this case we conclude that $j_{\ell-1}$ is a position in the semi-strict path $i_{0}<i_{1}<\cdots<i_{q-1}$ and, thus, $\left(\bar{w}, j_{\ell-1}\right) \models\left(\beta_{\psi} \vee\right.$ ret $)$ since $\left(\bar{w}, j_{\ell-1}\right) \models \psi$ and we assume that $\psi$ and $\beta_{\psi}$ are equivalent. It only remains to show that:

$$
\left(\bar{w}, j_{\ell-1}\right) \models\left(\neg \text { mcall } \rightarrow \bigcirc \beta_{\theta}\right) \wedge\left(\operatorname{mcall} \rightarrow\left(\bigcirc \beta_{\theta} \vee \bigcirc_{\mu} \bigcirc \beta_{\theta} \vee \bigcirc(\neg \text { ret } \wedge \gamma)\right)\right)
$$

If $j_{\ell-1}$ is a matched call position, then given that condition (I) does not hold, we have that $i_{q}=j_{\ell}=r\left(j_{\ell-1}\right)=j_{\ell-1}+1$. Thus, given that $\left(\bar{w}, i_{q}\right) \models \theta$ and we assume that $\theta$ and $\beta_{\theta}$ are equivalent, we conclude that $\left(\bar{w}, j_{\ell-1}\right) \models \bigcirc \beta_{\theta}$ and, therefore, $\left(\bar{w}, j_{\ell-1}\right) \models$ mcall $\rightarrow \bigcirc \beta_{\theta}$. If $j_{\ell-1}$ is not a matched call position, then we have that $i_{q}=j_{\ell}=$ $j_{\ell-1}+1$. Thus, given that $\left(\bar{w}, i_{q}\right) \models \theta$, we have that $\left(\bar{w}, j_{\ell-1}\right) \models \bigcirc \beta_{\theta}$ and, therefore, $\left(\bar{w}, j_{\ell-1}\right) \models \neg$ mcall $\rightarrow \bigcirc \beta_{\theta}$. This concludes the proof of Lemma 4.3. 